\title{
Multiplicative structures on the twisted equivariant K-theory of finite groups
}

\author{
César Galindo, Ismael Gutiérrez ${ }^{* *}$ and Bernardo Uribe
}

\begin{abstract}
Let $K$ be a finite group and let $G$ be a finite group acting on $K$ by automorphisms. In this paper we study two different but intimately related subjects: on the one side we classify all possible multiplicative and associative structures with which one can endow the twisted $G$-equivariant K-theory of $K$, and on the other, we classify all possible monoidal structures with which one can endow the category of twisted and $G$-equivariant bundles over $K$. We achieve this classification by encoding the relevant information in the cochains of a sub double complex of the double bar resolution associated to the semi-direct product $K \rtimes G$; we use known calculations of the cohomology of $K, G$ and $K \rtimes G$ to produce concrete examples of our classification.

In the case in which $K=G$ and $G$ acts by conjugation, the multiplication map $G \rtimes G \rightarrow G$ is a homomorphism of groups and we define a shuffle homomorphism which realizes this map at the homological level. We show that the categorical information that defines the Twisted Drinfeld Double can be realized as the dual of the shuffle homomorphism applied to any 3cocycle of $G$. We use the pullback of the multiplication map in cohomology to classify the possible ring structures that the Grothendieck ring of representations of the Twisted Drinfeld Double may have, and we include concrete examples of this procedure.
\end{abstract}

Mathematics Subject Classification (2010). 19L50, 18D10; 16L35, 20 J06.

Keywords. Twisted equivariant K-theory, twisted Drinfeld double, fusion category, multiplicative structure, fusion algebra.

\section{Introduction}

The purpose of this work is to investigate a relationship existing among certain tensor categories attached to a semi-direct product of groups (they include the Twisted

\footnotetext{
${ }^{*}$ C. G. would like to thank the hospitality of the Mathematics Department at MIT where part of this work was carried out. C. G. was partially supported by the FAPA funds from vicerrectoría de investigaciones de la Universidad de los Andes.

**I. G. acknowledges and thanks the financial support of the Deutscher Akademischer Austausch Dienst.

${ }^{*}$ B. U. acknowledges and thanks the financial support of the Alexander Von Humboldt Foundation.
} 
Drinfeld Double of a discrete group), their fusion algebras and the cohomology groups of the semi-direct product. These tensor categories, as well as some of our results, are closely related to abelian extensions of Hopf algebras and the cohomological description of Opext $\left(\mathbb{C}^{G}, \mathbb{C} F\right)$ ) given by Kac in [12], cf. [14].

The abelian extension theory of Hopf algebras was generalized to coquasi-Hopf algebras by Masuoka in [15]. Some of our results and constructions can be framed in the abelian extension theory of coquasi-Hopf algebras in the particular case where the matched pair of groups is a semi-direct product. However, our approach to these tensor categories does not follow Masuoka's point of view, instead we use the concept of pseudomonoids in a suitable 2-monoidal 2-category associated to a group. There are some reasons why we prefer to use the pseudomonoid approach: First, it exhibits more clearly the relationship between the cohomology of semi-direct products and the multiplicative structures of the $G$-equivariant twisted $K$-theory of a finite group $K$. Second, some terms of a spectral sequence associated to the double complex have a direct interpretation in terms of obstructions and classification of the possible pseudomonoid structures. Third, some of our constructions and results make sense in categories different from the category of sets; in particular, if we change to the Cartesian categories of locally compact topological spaces and change discrete group cohomology to the Borel-Moore cohomology [16], we have a more general theory where the categories of coquasi-Hopf algebras may not capture all the desired information.

There are two main reasons for our interest in the fusion algebra of the tensor categories defined in this paper. On the one side, semisimple tensor categories can be encoded in a combinatorial structure divided in two parts: the fusion algebra (or the Gorthendick ring of the tensor category) and some non-abelian cohomological information provided by the $F$-matrices of the $6 j$-symbols (see [18]). And on the other, these fusion algebras are generalizations of ${ }^{\omega} \mathrm{K}([G / G])$, the $w$-twisted stringy $K$-theory of the groupoid $[G / G]$ (see [19]).

In the case that the semi-direct product is finite, the associated tensor categories are fusion categories, and they belong to a bigger family of fusion categories called group-theoretical fusion categories for which many interesting results have been established cf. [9, 17]. Since an explicit description of the fusion rules of the tensor categories studied in this paper, via induction and restriction of projective representations of certain subgroups of $G$ already appear in [20, Theorem 4.8], our approach focuses in determining the number of fusion category and fusion algebra structures associated to a fixed semi-direct product. We accomplish this task in several steps. First, we show that the information encoding a pseudomonoid with strict unit in the 2-category of $G$-sets with twists over the group $K$ is equivalent to a 3-cocycle in $Z^{3}\left(\operatorname{Tot}^{*}\left(A^{*, *}(K \rtimes G, \mathbb{T})\right)\right)$, where the double complex $A^{*, *}(K \rtimes G, \mathbb{T})$ is the sub double complex without the 0 -th row of the double bar resolution $C^{*, *}(K \rtimes G, \mathbb{T})$ and whose total cohomology calculates the cohomology of $K \rtimes G$. Second, we show that the information encoded in a pseudomonoid with 
strict unit in the 2-category of $G$-sets with twists $\mathcal{K}$, is precisely the information required to endow the category $\operatorname{Bun}_{G}(\mathcal{K})$ of projective $G$-equivariant complex vector bundles with a monoidal structure; hence the isomorphism classes of bundles $\operatorname{Groth}\left(B u n_{G}(\mathcal{K})\right)$ becomes a fusion algebra, and this fusion algebra structure could be alternatively understood as a twisted $G$-equivariant K-theory ring of $K$. Third, we construct the twisted $G$-equivariant $\mathrm{K}$-theory of the group $K$ and we show the conditions under which this group could be endowed with a multiplicative structure making it a ring; we define the group of multiplicative structures by $M S_{G}(K)$ and we show that this group could be calculated by the use of a spectral sequence associated to the complex $\operatorname{Tot}^{*}\left(A^{*, *}(K \rtimes G, \mathbb{T})\right)$. We study the canonical homomorphism $H^{3}\left(\operatorname{Tot}^{*}\left(A^{*, *}(K \rtimes G, \mathbb{T})\right)\right) \stackrel{\phi}{\rightarrow} M S_{G}(K)$ and we give an explicit description of its kernel and its cokernel; a multiplicative structure on $M S_{G}(K)$ not appearing in the image of $\phi$, endows the twisted $G$-equivariant K-theory of $K$ with a ring structure which is not the fusion algebra of any tensor category of the form $B u n_{G}(\mathcal{K})$, in other words, it is an algebra structure which is not possible to categorize.

Of particular interest is the case in which $G=K$ and $G$ acts on itself by conjugation. In this case, the cohomological information which was used in [7] to define the Twisted Drinfeld Double $D^{w}(G)$ for $w \in Z^{3}(G, \mathbb{T})$ defines an element in $Z^{3}\left(\operatorname{Tot}^{*}\left(A^{*, *}(G \rtimes G, \mathbb{T})\right)\right)$. The formulæ defining this 3-cocycle were reminiscent of the formulæ appearing in [8, Theorem 5.2] on the proof of the EilenbergZilber theorem, and we conjectured that there had to exist a way to define for any cocycle in $Z^{n}(G, \mathbb{T})$ a $n$-cocycle in $Z^{n}\left(\operatorname{Tot}^{*}\left(A^{*, *}(G \rtimes G, \mathbb{T})\right)\right.$ ) having similar properties as the ones defined for $n=3$. We show in this paper that indeed this is the case and its proof is based on two facts: first that the multiplication map $\mu: G \rtimes G \rightarrow G, \mu(k, g)=k g$ is a homomorphism of groups, and second, on a construction of an explicit Shuffle homomorphism at the chain level, whose dual $\tau^{\vee}: C^{*}(G, \mathbb{T}) \rightarrow \operatorname{Tot}^{*}\left(C^{*, *}(G \rtimes G, \mathbb{T})\right)$ applied to $w$ recovers the cocycle defined in [7], and moreover that in cohomology equals the pullback of $\mu$, i.e. $\mu^{*}=\tau^{*}: H^{*}(G, \mathbb{T}) \rightarrow H^{*}(G \rtimes G, \mathbb{T})$. Since the group $G \rtimes G$ is isomorphic to the product $G \times G$ we get that the map $H^{3}\left(\operatorname{Tot}^{*}\left(A^{*, *}(G \rtimes G, \mathbb{T})\right)\right) \stackrel{\phi}{\rightarrow} M S_{G}(G)$ is surjective, and since we know that the cohomology class of the 3-cocycle that is defined in [7] could be recovered from the cohomology class of $\mu^{*} w$, we give a simple procedure to determine the fusion algebras of $\operatorname{Rep}\left(D^{w}(G)\right)$ which are isomorphic to the $G$-equivariant $\mathrm{K}$-theory ring $\mathbb{K U}_{G}(G)$; at the end of this work this procedure is exemplified in some interesting cases.

This paper is organized as follows. In Section 2 we provide background material on the semi-direct products and the double bar complex associated to its cohomology. In Section 3 the Shuffle homomorphism of a trivializable semidirect product is defined and some of its properties are shown. In Section 4 the 2-monoidal 2-category of twisted $G$-sets with strict unit and the 2-category of pseudomonoids in this 2-category are defined and described using the complex $\operatorname{Tot}^{*}\left(A^{*, *}(K \rtimes G, \mathbb{T})\right)$. In Section 5 the tensor category of equivariant vector bundles 
over a group, tensor functors and monoidal natural isomorphism associated to the 2category of pseudomonoids in the 2-category of twisted $G$-sets with strict unit are defined. In Section 6 the obstruction to the existence of multiplicative structures over the twisted $G$-equivariant $\mathrm{K}$-theory of a group $K$ is described using the spectral sequence associated to the filtration $F^{r}:=A^{*, *>r}$ of the double complex $A^{*, *}$. In Section 7 several concrete examples are completely calculated. We finish with an appendix in Section A in which we give the explicit relation of our tensor categories with coquasi-bialgebras.

\section{Preliminaries}

2.1. Semi-direct products. Let $K$ be a discrete group endowed with an action of the discrete group $G$ defined through a homomorphism $\rho: G \rightarrow \operatorname{Aut}(K)$; for simplicity, for $g \in G$ and $k \in K$ denote the action by $g(k):=\rho(g)(k)$. Denote by $K \rtimes G$ the group defined by the semi-direct product of $G$ with $K$; as a set $K \rtimes G:=$ $K \times G$ and the product structure is defined by $(a, g)(b, h):=(a g(b), g h)$.

The group $K \rtimes G$ fits in the short exact sequence

$$
1 \rightarrow K \rightarrow K \rtimes G \stackrel{\pi_{2}}{\rightarrow} G \rightarrow 1
$$

and we say that $K \rtimes G$ is isomorphic to another split extension

$$
1 \rightarrow K \rightarrow E \stackrel{p}{\rightarrow} G \rightarrow 1
$$

whenever there is an isomorphism $\psi: K \rtimes G \stackrel{\cong}{\rightrightarrows} E$ such that $\pi_{2}=p \circ \psi$.

Recall that $\operatorname{Inn}(K)$ is the group of inner automorphisms of the group $K$, i.e. the automorphisms of $K$ induced by conjugation, and that it fits in the short exact sequences

$$
\begin{gathered}
1 \rightarrow Z(K) \rightarrow K \stackrel{\tau}{\rightarrow} \operatorname{Inn}(K) \rightarrow 1 \\
1 \rightarrow \operatorname{Inn}(K) \rightarrow \operatorname{Aut}(K) \rightarrow \operatorname{Out}(K) \rightarrow 1
\end{gathered}
$$

where $Z(K)$ denotes the center of $K$ and $\operatorname{Out}(K)$ denotes the group of outer automorphisms of $K$.

Proposition 2.1. The exact sequence (2.1) of the semi-direct product $K \rtimes G$ is isomorphic to the trivial exact sequence of the product $K \times G$ if and only if the image of $\rho$ is in $\operatorname{Inn}(K)$, and there is a homomorphism $\sigma: G \rightarrow K$ such that the following diagram commutes

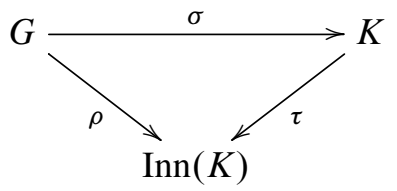

where the action of $G$ on $K$ is given by the inner automorphisms defined by $\rho$. 
Example 2.2. Let $K=G$ and consider the conjugation action of $K$ on itself. In this case $\rho=\tau$ and we can take $\sigma=\operatorname{Id}_{K}$. Therefore the map

$$
\mu: K \rtimes K \rightarrow K, \quad(a, g) \mapsto a g
$$

is a homomorphism of groups and

$$
\mu \times \pi_{2}: K \rtimes K \rightarrow K \times K, \quad(a, g) \mapsto(a g, g)
$$

is an isomorphism.

Remark 2.3. Note that whenever we have a homomorphism $\sigma: G \rightarrow K$ such that $\rho=\tau \circ \sigma$ then the map

$$
\mu: K \rtimes G \rightarrow K, \quad(k, g) \mapsto k \sigma(g)
$$

becomes a group homomorphism. Moreover, the map

$$
K \rtimes G \rightarrow K \rtimes K, \quad(k, g) \mapsto(k, \sigma(g))
$$

is a homomorphism of groups.

2.2. Bar resolution. Let us find an explicit model for the homology of the group $K \rtimes G$. For this, let us first setup the notation for the explicit model for the bar resolution that we will use.

Take $H$ a discrete group and define the complex $C_{*}(E H, \mathbb{Z})$ with

$$
C_{n}(E H, \mathbb{Z}):=\mathbb{Z} H^{\otimes n+1}
$$

and with differential $\partial_{H}: C_{n}(E H, \mathbb{Z}) \rightarrow C_{n-1}(E H, \mathbb{Z})$ defined by the equation on generators

$\partial_{H}\left(h_{1}, h_{2}, \ldots, h_{n+1}\right)=\left(h_{2}, h_{3}, \ldots, h_{n+1}\right)+\sum_{i=1}^{n}(-1)^{i}\left(h_{1}, \ldots, h_{i} h_{i+1}, \ldots, h_{n+1}\right)$.

The complex $\left(C_{*}(E H, \mathbb{Z}), \partial_{H}\right)$ becomes a complex in the category of $\mathbb{Z} H$-modules if we endow each $C_{n}(E H, \mathbb{Z})$ with the left $\mathbb{Z} H$-module structure defined by the equation

$$
h \cdot\left(h_{1}, \ldots, h_{n+1}\right):=\left(h_{1}, \ldots, h_{n+1} h^{-1}\right) .
$$

The augmentation map

$$
\epsilon: C_{0}(E H, \mathbb{Z}) \rightarrow \mathbb{Z}, \quad \epsilon(h)=1
$$

is a map of $\mathbb{Z} H$-modules and the complex $C_{*}(E H, \mathbb{Z})$ becomes a $\mathbb{Z} H$-free resolution of the trivial $\mathbb{Z} H$-module $\mathbb{Z}$,

$$
\epsilon: C_{*}(E H, \mathbb{Z}) \rightarrow \mathbb{Z} .
$$


The elements

$$
\left(h_{1}, h_{2}, \ldots, h_{n}, 1\right)
$$

generate the $\mathbb{Z} H$-module $C_{n}(E H, \mathbb{Z})$ and therefore we could write them using the "bar notation"

$$
\left[h_{1}\left|h_{2}\right| \ldots \mid h_{n}\right]:=\left(h_{1}, h_{2}, \ldots, h_{n}, 1\right) ;
$$

the differential $\partial_{H}$ in this base becomes

$$
\begin{aligned}
\partial_{H}\left[h_{1}\left|h_{2} \ldots\right| h_{n}\right]=\left[h_{2}\left|h_{3}\right| \ldots \mid h_{n}\right]+\sum_{i=1}^{n}(-1)^{i} & {\left[h_{1}|\ldots| h_{i} h_{i+1}|\ldots| h_{n}\right] } \\
& +(-1)^{n+1} h_{n}^{-1} \cdot\left[h_{1}|\ldots| h_{n-1}\right] .
\end{aligned}
$$

For a left $\mathbb{Z} H$-module $W$, the homology groups of $H$ with coefficients in $W$ are defined as

$$
H_{*}(H, W):=H_{*}\left(C_{*}(E H, \mathbb{Z}) \otimes_{\mathbb{Z} H} W\right)
$$

and the cohomology groups of $H$ with coefficients in $W$ as

$$
H^{*}(H, W):=H^{*}\left(\operatorname{Hom}_{\mathbb{Z} H}\left(C_{*}(E H, \mathbb{Z}), W\right)\right) .
$$

Since we have a canonical isomorphism of $\mathbb{Z}$-modules

$$
\operatorname{Hom}_{\mathbb{Z} H}\left(C_{n}(E H, \mathbb{Z}), W\right) \cong \operatorname{Maps}\left(H^{n}, W\right),
$$

the cohomological differential $\delta_{H}$ in terms of the bar notation becomes

$$
\begin{aligned}
\left(\delta_{H} f\right)\left[h_{1}\left|h_{2} \ldots\right| h_{n}\right]=f\left[h_{2}\left|h_{3}\right| \ldots \mid h_{n}\right]+ & \sum_{i=1}^{n}(-1)^{i} f\left[h_{1}|\ldots| h_{i} h_{i+1}|\ldots| h_{n}\right] \\
& +(-1)^{n+1} h_{n}^{-1} \cdot f\left[h_{1}|\ldots| h_{n-1}\right] .
\end{aligned}
$$

2.3. Cohomology of $K \rtimes G$. Consider the double complex

$$
C_{*}(E G, \mathbb{Z}) \otimes_{\mathbb{Z}} C_{*}(E K, \mathbb{Z})
$$

with differentials $\partial_{G} \otimes 1$ and $1 \otimes \partial_{K}$. Denote by $\left(g_{1}, \ldots, g_{p+1}|| k_{1}, \ldots, k_{q+1}\right)$ a generator in $C_{p}(E G, \mathbb{Z}) \otimes_{\mathbb{Z}} C_{q}(E K, \mathbb{Z})$ and define the action of $(k, g) \in K \rtimes G$ by the equation

$(k, g) \cdot\left(g_{1}, \ldots, g_{p+1} \| k_{1}, \ldots, k_{q+1}\right):=\left(g_{1}, \ldots, g_{p+1} g^{-1} \| g\left(k_{1}\right), \ldots, g\left(k_{q+1}\right) k^{-1}\right)$.

A straightforward computation shows that indeed it is an action and therefore $C_{p}(E G, \mathbb{Z}) \otimes_{\mathbb{Z}} C_{q}(E K, \mathbb{Z})$ becomes a free $\mathbb{Z}(K \rtimes G)$-module. Since the differentials $\partial_{G} \otimes 1$ and $1 \otimes \partial_{K}$ are also maps of $\mathbb{Z}(K \rtimes G)$-modules, we could take the total complex

$$
\operatorname{Tot}_{*}\left(C_{*}(E G, \mathbb{Z}) \otimes_{\mathbb{Z}} C_{*}(E K, \mathbb{Z})\right)
$$


whose degree $n$-component is

$$
\operatorname{Tot}_{n}\left(C_{*}(E G, \mathbb{Z}) \otimes_{\mathbb{Z}} C_{*}(E K, \mathbb{Z})\right):=\bigoplus_{p+q=n} C_{p}(E G, \mathbb{Z}) \otimes_{\mathbb{Z}} C_{q}(E K, \mathbb{Z})
$$

and whose differential is

$$
\partial_{G} \otimes 1 \oplus(-1)^{p} 1 \otimes \partial_{K}
$$

thus obtaining a free $\mathbb{Z}(K \rtimes G)$ complex. Since the homology of the total complex of this double complex is just $\mathbb{Z}$ in degree 0 ,

$$
H_{*}\left(\operatorname{Tot}_{*}\left(C_{*}(E G, \mathbb{Z}) \otimes_{\mathbb{Z}} C_{*}(E K, \mathbb{Z})\right), \partial_{G} \otimes 1 \oplus(-1)^{p} 1 \otimes \partial_{K}\right)=\mathbb{Z},
$$

we have that

$$
\operatorname{Tot}_{*}\left(C_{*}(E G, \mathbb{Z}) \otimes_{\mathbb{Z}} C_{*}(E K, \mathbb{Z})\right) \stackrel{\epsilon \otimes \epsilon}{\longrightarrow} \mathbb{Z}
$$

is a free $\mathbb{Z}(K \rtimes G)$ resolution of $\mathbb{Z}$.

Making use of the bar notation we take the elements

$$
\left[g_{1}|\ldots| g_{p}|| k_{1}|\ldots| k_{q}\right]:=\left(g_{1}, \ldots, g_{p}, 1|| k_{1}, \ldots, k_{q}, 1\right)
$$

as a set of generators of $C_{p}(E G, \mathbb{Z}) \otimes_{\mathbb{Z}} C_{q}(E K, \mathbb{Z})$ as a $\mathbb{Z}(K \rtimes G)$-module; in this base we have the equality

$$
\left(g_{1}, \ldots, g_{p}, g|| k_{1}, \ldots, k_{q}, k\right)=\left(k^{-1}, g^{-1}\right) \cdot\left[g_{1}|\ldots| g_{p}|| g\left(k_{1}\right)|\ldots| g\left(k_{q}\right)\right] \text {. }
$$

This choice of base provides us with an isomorphism of $\mathbb{Z}$-modules

$$
\operatorname{Hom}_{\mathbb{Z}(K \rtimes G)}\left(C_{p}(E G, \mathbb{Z}) \otimes_{\mathbb{Z}} C_{q}(E K, \mathbb{Z}), \mathbb{Z}\right) \cong \operatorname{Maps}\left(G^{p} \times K^{q}, \mathbb{Z}\right)
$$

that allows us to transport the dual of the differentials $\partial_{G} \otimes 1$ and $1 \otimes \partial_{K}$ to $\operatorname{Maps}\left(G^{p} \times K^{q}, \mathbb{Z}\right)$; the induced differentials will be denote by $\delta_{G}$ and $\delta_{K}$. Therefore we obtain:

Definition 2.4. Let $\bar{C}^{p, q}(K \rtimes G, \mathbb{Z}), p, q \geq 0$, be the double complex

$$
\bar{C}^{p, q}(K \rtimes G, \mathbb{Z}):=\operatorname{Maps}\left(G^{p} \times K^{q}, \mathbb{Z}\right)
$$

with differentials $\delta_{G}: \bar{C}^{p, q} \rightarrow \bar{C}^{p+1, q}$ and $\delta_{K}: \bar{C}^{p, q} \rightarrow \bar{C}^{p, q+1}$ defined by the equations

$$
\begin{gathered}
\left(\delta_{G} F\right)\left[g_{1}|\ldots| g_{p+1}|| k_{1}|\ldots| k_{q}\right]=F\left[g_{2}|\ldots| g_{p+1}|| k_{1}|\ldots| k_{q}\right] \\
+\sum_{i=1}^{p}(-1)^{i} F\left[g_{1}|\ldots| g_{i} g_{i+1}|\ldots| g_{p+1}|| k_{1}|\ldots| k_{q}\right] \\
+(-1)^{p+1} F\left[g_{1}|\ldots| g_{p}|| g_{p+1}\left(k_{1}\right)|\ldots| g_{p+1}\left(k_{q}\right)\right] \\
\left(\delta_{K} F\right)\left[g_{1}|\ldots| g_{p}|| k_{1}|\ldots| k_{q+1}\right]=F\left[g_{1}|\ldots| g_{p}|| k_{2}|\ldots| k_{q+1}\right] \\
+\sum_{j=1}^{q}(-1)^{j} F\left[g_{1}|\ldots| g_{p}|| k_{1}|\ldots| k_{j} k_{j+1}|\ldots| k_{q+1}\right] \\
+(-1)^{q+1} F\left[g_{1}|\ldots| g_{p}|| k_{1}|\ldots| k_{q}\right],
\end{gathered}
$$

where by convention $G^{0} \times K^{0}$ is the set with one point. 
Hence we have

Lemma 2.5. The cohomology of the total complex of the double complex $\bar{C}^{*, *}(K \rtimes G$, $\mathbb{Z}), \delta_{G}, \delta_{K}$ is the cohomology of the group $K \rtimes G$, i.e.

$$
H^{*}\left(\operatorname{Tot}^{*}\left(\bar{C}^{*, *}(K \rtimes G, \mathbb{Z})\right), \delta_{G} \oplus(-1)^{p} \delta_{K}\right) \cong H^{*}(K \rtimes G, \mathbb{Z}) .
$$

We can take a smaller double complex, more suited for our work, which is called the normalized double complex. Let us define it

Definition 2.6. The normalized double complex of $\bar{C}^{*, *}(K \rtimes G, \mathbb{Z}), \delta_{G}, \delta_{K}$ is the double complex

$$
C^{p, q}(K \rtimes G, \mathbb{Z}) \subset \bar{C}^{p, q}(K \rtimes G, \mathbb{Z})
$$

consisting of maps $F: G^{p} \times K^{q} \rightarrow \mathbb{Z}$ such that $F\left[g_{1}|\ldots| g_{p}|| k_{1}|..| k_{q}\right]=1$ whenever $g_{i}=1$ or $k_{j}=1$. The differentials on $C^{p, q}$ are also $\delta_{G}$ and $\delta_{K}$. We setup $C^{0,0}=\mathbb{Z}$.

It is known in homological algebra that the normalized complex of the bar resolution is quasi-isomorphic to the bar resolution (see page 215 in [10]). Therefore we have

Lemma 2.7. The induced map on total complexes

$$
\operatorname{Tot}^{*}\left(C^{*, *}(K \rtimes G, \mathbb{Z})\right) \rightarrow \operatorname{Tot}^{*}\left(\bar{C}^{*, *}(K \rtimes G, \mathbb{Z})\right)
$$

is a quasi-isomorphism. Then

$$
H^{*}\left(\operatorname{Tot}^{*}\left(C^{*, *}(K \rtimes G, \mathbb{Z})\right), \delta_{G} \oplus(-1)^{p} \delta_{K}\right) \cong H^{*}(K \rtimes G, \mathbb{Z}) .
$$

We can generalize our definition of the double complex to other coefficients. Denote by $\mathbb{T}$ the group $S^{1}$ and consider the exact sequence of coefficients

$$
0 \longrightarrow \mathbb{Z} \longrightarrow \mathbb{R} \longrightarrow \mathbb{T} \longrightarrow 0 .
$$

We will denote the complex $C^{p, q}(K \rtimes G, \mathbb{T})$ as the complex generated by maps $F: G^{p} \times K^{q} \rightarrow \mathbb{T}$ on which $F$ is 1 if one of the entries is the identity, and the differentials $\delta_{G}$ and $\delta_{K}$ are the same ones as Definition 2.4 but changing the sums by multiplications. Note also that we set up $C^{0,0}(K \rtimes G, \mathbb{T}):=\mathbb{T}$.

Then

$$
H^{*}\left(\operatorname{Tot}^{*}\left(C^{*, *}(K \rtimes G, \mathbb{T})\right), \delta_{G} \oplus \delta_{K}^{(-1)^{p}}\right) \cong H^{*}(K \rtimes G, \mathbb{T}) .
$$

2.3.1. Decomposition of the cohomology of $K \rtimes G$. Note that the 0 -th row of the double complex is isomorphic to the normalized bar cochain complex of $G$

$$
\left(C^{*, 0}(K \rtimes G, \mathbb{Z}), \delta_{G}\right) \cong\left(C^{*}(G, \mathbb{Z}), \delta_{G}\right),
$$

and the action of the differential $\delta_{K}$ on this row is trivial. Therefore, if we define 
Definition 2.8. Let

$$
A^{*, *}(K \rtimes G, \mathbb{Z}):=C^{*, *>0}(K \rtimes G, \mathbb{Z})
$$

be the sub double complex of $C^{*, *}(K \rtimes G, \mathbb{Z})$ with trivial 0-th row, and differentials $\delta_{G}$ and $\delta_{K}$.

Then we obtain

Lemma 2.9. There is a canonical isomorphism of double complexes

$$
\begin{aligned}
\left(C^{*, *}(K \rtimes G, \mathbb{Z}), \delta_{G}\right. & \left.\oplus(-1)^{p} \delta_{K}\right) \\
& \cong\left(A^{*, *}(K \rtimes G, \mathbb{Z}), \delta_{G} \oplus(-1)^{p} \delta_{K}\right) \oplus\left(C^{*}(G, \mathbb{Z}), \delta_{G}\right),
\end{aligned}
$$

which induces a canonical isomorphism in cohomology

$$
H^{*}(K \rtimes G, \mathbb{Z}) \cong H^{*}\left(\operatorname{Tot}^{*}\left(A^{*, *}(K \rtimes G, \mathbb{Z})\right)\right) \oplus H^{*}(G, \mathbb{Z}),
$$

and the respective one with coefficients in $\mathbb{T}$,

$$
H^{*}(K \rtimes G, \mathbb{T}) \cong H^{*}\left(\operatorname{Tot}^{*}\left(A^{*, *}(K \rtimes G, \mathbb{T})\right)\right) \oplus H^{*}(G, \mathbb{T})
$$

where $A^{*, *}(K \rtimes G, \mathbb{T})=C^{*, *>0}(K \rtimes G, \mathbb{T})$.

\section{The case of the trivializable semi-direct product $K \rtimes G$}

Whenever the semi-direct product $K \rtimes G$ is isomorphic to $K \times G$ we know by Proposition 2.1 and Remark 2.3 that there exists a homomorphism $\sigma: G \rightarrow K$ such that $K \rtimes G \rightarrow K \rtimes K,(k, g) \mapsto(k, \sigma(g))$ is a homomorphism of groups. In this case we have the homomorphisms

$$
K \rtimes G \rightarrow K \rtimes K \stackrel{\mu}{\rightarrow} K, \quad(k, g) \mapsto(k, \sigma(g)) \mapsto k \sigma(g)
$$

which induce the following homomorphism at the level of their homologies

$$
H_{*}(K \rtimes G, \mathbb{Z}) \rightarrow H_{*}(K \rtimes K, \mathbb{Z}) \stackrel{\mu_{*}}{\rightarrow} H_{*}(K, \mathbb{Z}) .
$$

Since the interesting information lies on the homomorphism $\mu_{*}$ we will investigate its properties.

3.1. The Shuffle homomorphism. In what follows we will describe how to obtain the map $\mu_{*}$ at the chain level using the explicit models for $H_{*}(K \rtimes K, \mathbb{Z})$ and $H_{*}(K, \mathbb{Z})$ defined previously. Its definition needs some preparation. 
Take a base element $\left[g_{1}|\ldots| g_{p}|| k_{1}|\ldots| k_{q}\right]$ in $C_{p}(E K, \mathbb{Z}) \otimes_{\mathbb{Z}} C_{q}(E K, \mathbb{Z})$ and think of it as a way to represent $\Delta^{p} \times \Delta^{q}$ as the product of the simplices $\left[g_{1}|\ldots| g_{p}\right] \times$ $\left[k_{1}|\ldots| k_{q}\right]$ in $B K$. For $\lambda \in \operatorname{Shuff}(p, q)$ a $(p, q)$-shuffle, i.e. an element in the symmetric group $\mathfrak{S}_{p+q}$ such that $\lambda(i)<\lambda(j)$ whenever $1 \leq i<j \leq p$ or $p+1 \leq i<j \leq p+q$, we can define an element

$$
\lambda\left[g_{1}|\ldots| g_{p}\left|k_{1}\right| \ldots \mid k_{q}\right] \in C_{p+q}(E K, \mathbb{Z})
$$

such that $\lambda\left[g_{1}|\ldots| g_{p}\left|k_{1}\right| \ldots \mid k_{q}\right]:=\left[s_{1}|\ldots| s_{p+q}\right]$ with

$$
s_{\lambda(i)}=\left\{\begin{array}{cc}
g_{i} & \text { if } i \leq p \\
g_{p+\lambda(i)-i+1} \ldots g_{p-1} g_{p} k_{i-p}\left(g_{p+\lambda(i)-i+1} \ldots g_{p-1} g_{p}\right)^{-1} & \text { if } i>p .
\end{array}\right.
$$

From the Eilenberg-Zilber theorem it follows that the set

$$
\left\{\lambda\left[g_{1}|\ldots| g_{p}\left|k_{1}\right| \ldots \mid k_{q}\right]: \lambda \in \operatorname{Shuff}(p, q)\right\}
$$

is a subdivision in simplices of dimension $p+q$ of the product of the simplices $\left[g_{1}|\ldots| g_{p}\right] \times\left[k_{1}|\ldots| k_{q}\right]$.

An equivalent way to see the elements $\lambda\left[g_{1}|\ldots| g_{p}\left|k_{1}\right| \ldots \mid k_{q}\right]$ is the following: a $(p, q)$-shuffle can also be understood as a way to parameterize a lattice path of minimum distance from the point $(0,0)$ to the point $(p, q)$; one moves one unit to the right in the steps $\lambda(1), \ldots, \lambda(p)$ and one unit up in the steps $\lambda(p+1), \ldots, \lambda(p+q)$. We label the horizontal and vertical edges in the rectangular lattice defined by the points $(0,0),(p, 0),(p, q),(0, q)$ by the following rule:

- The horizontal path between $(i-1, j)$ and $(i, j)$ is labeled with $g_{i}$ independent of $j$.

- The vertical path between $(p, j-1)$ and $(p, j)$ is labeled with $k_{j}$ and all the other vertical paths are labeled in such a way that the squares become commutative squares (when thinking of the labels as maps). This implies that the vertical edge from $(i, j-1)$ to $(i, j)$ is labeled with

$$
g_{i+1} \ldots g_{p-1} g_{p} k_{j}\left(g_{i+1} \ldots g_{p-1} g_{p}\right)^{-1} .
$$

Since $\lambda$ parameterizes a path in the lattice, the element $\lambda\left[g_{1}|\ldots| g_{p}\left|k_{1}\right| \ldots \mid k_{q}\right]$ encodes the labels that the path $\lambda$ follow in order to go from $(0,0)$ to $(p, q)$.

We can now define the map of complexes which in homology realizes $\mu_{*}$ :

Definition 3.1. Let

$$
\tau: C_{p}(E K, \mathbb{Z}) \otimes_{\mathbb{Z}} C_{q}(E K, \mathbb{Z}) \rightarrow C_{p+q}(E K, \mathbb{Z})
$$

be the graded homomorphism defined on generators by the equation

$$
\begin{aligned}
\tau\left(g_{1}, \ldots g_{p}, g|| k_{1}, \ldots, k_{q}, k\right) & \\
& :=\sum_{\lambda \in \operatorname{Shuff}}(g, q)
\end{aligned}
$$


where $|\lambda|$ denotes the sign of the permutation $\lambda$. This is usually called the Shuffle homomorphism.

We claim that the Shuffle homomorphism $\tau$ is an admissible product in the sense described in [6, IV-55, p. 118]

Theorem 3.2. The graded homomorphism

$$
\tau: \operatorname{Tot}_{*}\left(C_{*}(E K, \mathbb{Z}) \otimes_{\mathbb{Z}} C_{*}(E K, \mathbb{Z})\right) \rightarrow C_{*}(E K, \mathbb{Z})
$$

is a chain map that satisfies the equations

$$
\begin{aligned}
\tau\left(\left(a^{-1}, h^{-1}\right) \cdot\left(g_{1}, \ldots g_{p}, g \| k_{1}, \ldots, k_{q}, k\right)\right) & =(h a)^{-1} \cdot \tau\left(g_{1}, \ldots g_{p}, g \| k_{1}, \ldots, k_{q}, k\right) \\
(\epsilon \otimes \epsilon)(k, g) & =\epsilon(k g) .
\end{aligned}
$$

Hence it induces a graded homomorphism in homology

$$
\tau_{*}: H_{*}(K \rtimes K, \mathbb{Z}) \rightarrow H_{*}(K, \mathbb{Z})
$$

which is equal to the one induced by the pushforward $\mu_{*}$.

Proof. A routine calculation shows that the equations above are satisfied. The proof of the fact that $\tau$ is a chain map, namely that

$$
\partial_{K} \circ \tau=\tau \circ\left(\partial_{K} \otimes 1+(-1)^{p} 1 \otimes \partial_{K}\right),
$$

is essentially included in the proof of [8, Theorem 5.2]; the decomposition in simplices of dimension $p+q$ of the product of the simplices $\left[g_{1}|\ldots| g_{p}\right] \times$ $\left[k_{1}|\ldots| k_{q}\right]$ in $B K$ is done by choosing appropriately $p+q$ edges with the use of the $(p, q)$-shuffles as follows

$$
\tau\left[g_{1}|\ldots| g_{p}|| k_{1}|\ldots| k_{q}\right]=\sum_{\lambda \in \operatorname{Shuff}(p, q)}(-1)^{\lambda} \lambda\left[g_{1}|\ldots| g_{p}\left|k_{1}\right| \ldots \mid k_{q}\right] .
$$

With this decomposition of $\left[g_{1}|\ldots| g_{p}\right] \times\left[k_{1}|\ldots| k_{q}\right]$, its boundary can be calculated as $\left(\partial_{K} \times 1+(-1)^{p} 1 \times \partial_{K}\right)\left[g_{1}|\ldots| g_{p}\right] \times\left[k_{1}|\ldots| k_{q}\right]$ or alternatively as $\partial_{K}\left(\tau\left[g_{1}|\ldots| g_{p}|| k_{1}|\ldots| k_{q}\right]\right)$; then it follows that $\tau$ is a chain map.

Now, since $\tau$ is a chain map which preserves the module structures, then it induces a chain map at the level of the coinvariants

$$
\operatorname{Tot}_{*}\left(C_{*}(E K, \mathbb{Z}) \otimes_{\mathbb{Z}} C_{*}(E K, \mathbb{Z})\right) \otimes_{\mathbb{Z}(K \rtimes K)} \mathbb{Z} \rightarrow C_{*}(E K, \mathbb{Z}) \otimes_{\mathbb{Z} K} \mathbb{Z}
$$

which defines a homomorphism

$$
\tau_{*}: H_{*}(K \rtimes K, \mathbb{Z}) \rightarrow H_{*}(K, \mathbb{Z}) .
$$

This homomorphism $\tau_{*}$ must be equal to the pushforward $\mu_{*}$ since $\tau$ preserves the module structures defined by $\mu$. 
If we have a homomorphism of groups $\sigma: G \rightarrow K$ for which the map $\psi: K \rtimes$ $G \stackrel{\cong}{\rightrightarrows} K \times G,(k, g) \mapsto(k, \sigma(g))$ induces an isomorphism of semi-direct products, then we have that the map

$$
\begin{aligned}
\tau \circ(\sigma \otimes 1): C_{p}(E G, \mathbb{Z}) \otimes_{\mathbb{Z}} C_{q}(E K, \mathbb{Z}) & \rightarrow C_{p+q}(E K, \mathbb{Z}) \\
{\left[g_{1}|\ldots| g_{p}|| k_{1}|\ldots| k_{q}\right] } & \mapsto \tau\left[\sigma\left(g_{1}\right)|\ldots| \sigma\left(g_{p}\right)|| k_{1}|\ldots| k_{q}\right]
\end{aligned}
$$

induces a chain map

$$
\tau \circ(\sigma \otimes 1): \operatorname{Tot}_{*}\left(C_{*}(E G, \mathbb{Z}) \otimes_{\mathbb{Z}} C_{*}(E K, \mathbb{Z})\right) \rightarrow C_{*}(E K, \mathbb{Z})
$$

preserving their respective module structures, and hence inducing a homomorphism

$$
(\tau \circ(\sigma \otimes 1))_{*}: H_{*}(K \rtimes G, \mathbb{Z}) \rightarrow H_{*}(K, \mathbb{Z})
$$

which is equal to the pushforward map of the composition $\mu \circ \psi$; i.e.

$$
(\mu \circ \psi)_{*}=(\tau \circ(\sigma \otimes 1))_{*}: H_{*}(K \rtimes G, \mathbb{Z}) \rightarrow H_{*}(K, \mathbb{Z}) .
$$

3.2. The dual of the Shuffle homomorphism. Dualizing the map $\tau$, we obtain a homomorphism

$$
\begin{aligned}
\left.\tau^{\vee}: \bar{C}^{n}(K, \mathbb{Z})\right) & \rightarrow \bigoplus_{p+q=n} \bar{C}^{p, q}(K \rtimes K, \mathbb{Z}) \\
\left(\tau^{\vee} F\right)\left[s_{1}|\ldots| s_{n}\right] & \mapsto \bigoplus_{p+q=n} F\left(\tau\left[s_{1}|\ldots| s_{p}|| s_{p+1}|\ldots| s_{p+q}\right]\right)
\end{aligned}
$$

which induces a cochain map

$$
\tau^{\vee}:\left(\bar{C}^{*}(K, \mathbb{Z}), \delta_{K}\right) \rightarrow\left(\operatorname{Tot}^{*}\left(\bar{C}^{*, *}(K \rtimes K, \mathbb{Z})\right), \delta_{G} \oplus(-1)^{p} \delta_{K}\right) ;
$$

here we have kept the notation of the differentials as $\delta_{G}$ and $\delta_{K}$ in order to avoid confusion. A straightforward calculation shows that the cochain map $\tau^{\vee}$ preserves normalized cochains

$$
\tau^{\vee}:\left(C^{*}(K, \mathbb{Z}), \delta_{K}\right) \rightarrow\left(\operatorname{Tot}^{*}\left(C^{* * *}(K \rtimes K, \mathbb{Z})\right), \delta_{G} \oplus(-1)^{p} \delta_{K}\right)
$$

and therefore it induces a homomorphism at the level of cohomologies

$$
\tau^{*}: H^{*}(K ; \mathbb{Z}) \rightarrow H^{*}(K \rtimes K, \mathbb{Z})
$$

which by Theorem 3.2 is equal to the pullback of the homomorphism of groups $\mu$. 
If we bundle up our previous discussion we have

Theorem 3.3. The homomorphism in cohomology

$$
\tau^{*}: H^{*}(K, \mathbb{Z}) \rightarrow H^{*}(K \rtimes K, \mathbb{Z})
$$

defined by the cochain map

$$
\begin{aligned}
\left.\tau^{\vee}: C^{n}(K, \mathbb{Z})\right) & \rightarrow \bigoplus_{p+q=n} C^{p, q}(K \rtimes K, \mathbb{Z}) \\
\left(\tau^{\vee} F\right)\left[s_{1}|\ldots| s_{n}\right] & \mapsto \bigoplus_{p+q=n} F\left(\tau\left[s_{1}|\ldots| s_{p}|| s_{p+1}|\ldots| s_{p+q}\right]\right)
\end{aligned}
$$

is equal to

$$
\mu^{*}: H^{*}(K, \mathbb{Z}) \rightarrow H^{*}(K \rtimes K, \mathbb{Z})
$$

which is the pullback of the group homomorphism $\mu: K \rtimes K \rightarrow K,(k, g) \mapsto k g$.

3.3. Further properties of the Shuffle homomorphism. Consider the homomorphisms

$$
\begin{aligned}
\iota_{K}: K & \rightarrow K \rtimes K, & \iota_{G}: K & \rightarrow K \rtimes K \\
x & \mapsto\left(x, 1_{K}\right) & g & \mapsto\left(1_{K}, g\right) .
\end{aligned}
$$

and note that they fit into the commutative diagram

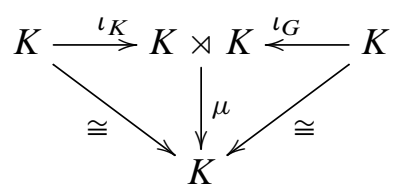

which induces the commutative diagram

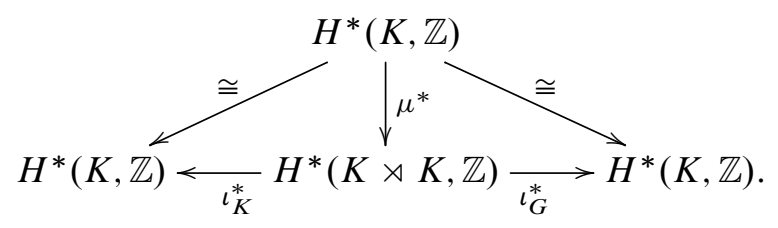

From Lemma 2.9 we know that there is a canonical isomorphism

$$
H^{p}(K \rtimes K, \mathbb{Z}) \cong H^{p}\left(\operatorname{Tot}^{*}\left(A^{*, *}(K \rtimes K, \mathbb{Z})\right)\right) \oplus H^{p}(K, \mathbb{Z})
$$

and the homomorphism $\iota_{G}^{*}$ is precisely the projection on the second component of the direct sum $H^{p}\left(\operatorname{Tot}^{*}\left(A^{*, *}(K \rtimes K, \mathbb{Z})\right)\right) \oplus H^{p}(K, \mathbb{Z})$. 
Moreover, by defining the chain map

$$
\begin{aligned}
\left.\tau_{1}^{\vee}: C^{n}(K, \mathbb{Z})\right) & \rightarrow \bigoplus_{p+q=n, q>0} A^{p, q}(K \rtimes K, \mathbb{Z}) \\
\left(\tau_{1}^{\vee} F\right)\left[s_{1}|\ldots| s_{n}\right] & \mapsto \bigoplus_{p+q=n, q>0} F\left(\tau\left[s_{1}|\ldots| s_{p}|| s_{p+1}|\ldots| s_{p+q}\right]\right)
\end{aligned}
$$

we have that the chain map

$$
\left.\tau^{\vee}: C^{*}(K, \mathbb{Z})\right) \rightarrow \operatorname{Tot}^{*}\left(A^{*, *}(K \rtimes K, \mathbb{Z})\right) \oplus C^{*, 0}(K \rtimes K, \mathbb{Z})
$$

is isomorphic to the chain map

$$
\left.\tau_{1}^{\vee} \oplus 1: C^{*}(K, \mathbb{Z})\right) \rightarrow \operatorname{Tot}^{*}\left(A^{*, *}(K \rtimes K, \mathbb{Z})\right) \oplus C^{*, 0}(K \rtimes K, \mathbb{Z})
$$

and therefore we have that at the cohomological level we obtain the commutative diagram

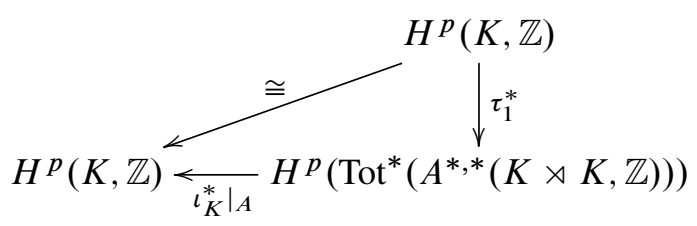

for all $p>0$, where at the cochain level $\left.\iota_{K}^{*}\right|_{A}$ is simply the projection map on the 0 -th column

$$
\left.\iota_{K}^{*}\right|_{A}: \operatorname{Tot}^{*}\left(A^{*, *}(K \rtimes K, \mathbb{Z})\right) \rightarrow A^{*, 0}(K \rtimes K, \mathbb{Z}) \cong C^{*>0}(K, \mathbb{Z}) .
$$

Therefore we make the following definition

Definition 3.4. Let

$$
B^{*, *}(K \rtimes G, \mathbb{Z}):=C^{*>0, *>0}(K \rtimes G, \mathbb{Z})
$$

be the sub double complex of $C^{*, *}(K \rtimes G, \mathbb{Z})$ with trivial 0-th row and trivial 0-th column, and differentials $\delta_{G}$ and $\delta_{K}$.

Lemma 3.5. The homomorphism

$$
\begin{aligned}
H^{p}(K, \mathbb{Z}) \oplus H^{p}\left(\operatorname{Tot}^{*}\left(B^{*, *}(K \rtimes K, \mathbb{Z})\right)\right) & \rightarrow H^{p}\left(\operatorname{Tot}^{*}\left(A^{*, *}(K \rtimes K, \mathbb{Z})\right)\right) \\
x \oplus y & \mapsto \tau_{1}^{*} x+y
\end{aligned}
$$

is an isomorphism for all $p>0$.

Proof. The short exact sequence of complexes

$$
0 \rightarrow \operatorname{Tot}^{*}\left(B^{*, *}(K \rtimes K, \mathbb{Z})\right) \rightarrow \operatorname{Tot}^{*}\left(A^{*, *}(K \rtimes K, \mathbb{Z})\right) \rightarrow C^{*>0}(K, \mathbb{Z}) \rightarrow 0
$$


induces a long exact sequence in cohomology groups

$\rightarrow H^{p}\left(\operatorname{Tot}^{*}\left(B^{*, *}(K \rtimes K, \mathbb{Z})\right)\right) \rightarrow H^{p}\left(\operatorname{Tot}^{*}\left(A^{*, *}(K \rtimes K, \mathbb{Z})\right)\right) \stackrel{\left.\iota_{K}^{*}\right|_{A}}{\rightarrow} H^{p}(K, \mathbb{Z}) \rightarrow$

which splits by diagram (3.3).

Therefore we can conclude that there is a canonical splitting of the cohomology of $K \rtimes K$ in terms of the cohomology of $K$ and of the cohomology of the double complex $B^{*, *}(K \rtimes K, \mathbb{Z})$.

Proposition 3.6. The homomorphism

$$
\begin{aligned}
& H^{p}(K, \mathbb{Z}) \oplus H^{p}\left(\operatorname{Tot}^{*}\left(B^{*, *}(K \rtimes K, \mathbb{Z})\right)\right) \oplus H^{p}(K, \mathbb{Z}) \stackrel{\cong}{\rightarrow} H^{p}(K \rtimes K, \mathbb{Z}) \\
& x \oplus y \oplus z \mapsto \tau_{1}^{*} x+y+\pi_{2}^{*} z
\end{aligned}
$$

is an isomorphism for all $p>0$. Here $\pi_{2}: K \rtimes K \rightarrow K,(a, g) \mapsto g$ denotes the homomorphism induced by the projection on the second coordinate satisfying $\pi_{2} \circ \iota_{G}=1$.

\section{Categorical definitions}

4.1. 2-category of discrete $G$-sets with twist. We shall fix a discrete group $G$. We define the 2-category of discrete $G$-sets with twist as follows:

(1) Objects will be called discrete $G$-sets with twist, and they are pairs $(X, \alpha)$, where $X$ is a discrete left $G$-set and $\alpha \in Z_{G}^{2}(X, \mathbb{T})$ is a normalized 2-cocycle in the $G$-equivariant complex of $X$, i.e. a map

$$
\alpha: G \times G \times X \rightarrow \mathbb{T},
$$

such that

$$
\alpha[\tau|\rho| \mid x] \alpha[\sigma \tau \mid \rho \| x]^{-1} \alpha[\sigma \mid \tau \rho \| x] \alpha[\sigma|\tau| \mid \rho x]^{-1}=1
$$

for all $\sigma, \tau, \rho \in G, x \in X$.

Note that the previous equation is equivalent to the equation $\delta_{G} \alpha=1$, when we see $\alpha$ as element in $C^{2,1}(X \rtimes G, \mathbb{T})$.

(2) Let $\left(X, \alpha_{X}\right),\left(Y, \alpha_{Y}\right)$ be discrete $G$-sets with twist. A 1-cell from $\left(X, \alpha_{X}\right)$ to $\left(Y, \alpha_{Y}\right)$, also called a $G$-equivariant map, is a pair $(L, \beta)$,

$$
\left(X, \alpha_{X}\right) \stackrel{(L, \beta)}{\longrightarrow}\left(Y, \alpha_{Y}\right)
$$


where

- $L: X \rightarrow Y$ is a morphism of $G$-sets,

- $\beta \in C_{G}^{1}(X, \mathbb{T})$ is a normalized cochain such that $\delta_{G}(\beta)=L^{*}\left(\alpha_{Y}\right)\left(\alpha_{X}\right)^{-1}$, i.e., a map

$$
\beta: G \times X \rightarrow \mathbb{T}
$$

such that

$$
\beta[\tau|| x] \beta[\sigma \tau \mid x]^{-1} \beta[\sigma \| \tau x]=\alpha_{Y}[\sigma|\tau| \mid L(x)] \alpha_{X}[\sigma|\tau| \mid x]^{-1}
$$

for all $\sigma, \tau \in G, x \in X$.

(3) Given two 1-cells $(L, \beta),\left(L, \beta^{\prime}\right):\left(X, \alpha_{X}\right) \rightarrow\left(Y, \alpha_{Y}\right)$, a 2-cell $\theta:(L, \beta) \Rightarrow$ $\left(L, \beta^{\prime}\right)$

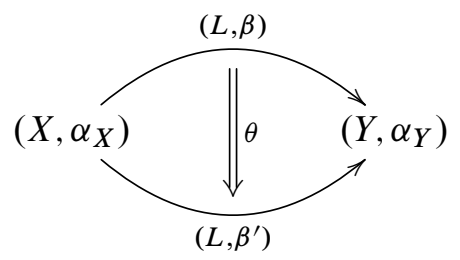

is 0-cochain $\theta \in C_{G}^{0}(X, \mathbb{T})$ such that $\delta_{G}(\theta)=\beta^{\prime} \beta^{-1}$, i.e., a map $\theta: X \rightarrow \mathbb{T}$, such that

$$
\theta[x] \theta[\sigma x]^{-1}=\beta^{\prime}[\sigma \| x] \beta[\sigma \| x]^{-1}
$$

for all $\sigma \in G, x \in X$.

Let us define the composition on 1-cells and 2-cells in the following way. Let $\left(F, \beta_{F}\right):\left(X, \alpha_{X}\right) \rightarrow\left(Y, \alpha_{Y}\right)$ and $\left(G, \beta_{G}\right):\left(Y, \alpha_{Y}\right) \rightarrow\left(Z, \alpha_{Z}\right)$ two 1-cells, define their composition as

$$
\left(G, \beta_{G}\right) \circ\left(F, \beta_{F}\right)=\left(G \circ F, F^{*}\left(\beta_{G}\right) \beta_{F}\right):\left(X, \alpha_{X}\right) \rightarrow\left(Z, \alpha_{Z}\right),
$$

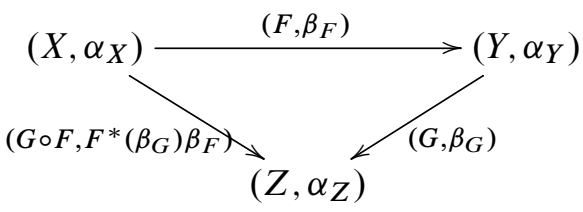

and if $\theta:(L, \beta) \Rightarrow\left(L, \beta^{\prime}\right)$ and $\theta^{\prime}:\left(L, \beta^{\prime}\right) \Rightarrow\left(L, \beta^{\prime \prime}\right)$ are 2-cells, their composition is the product of the maps, namely

$$
\theta^{\prime} \circ \theta=: \theta^{\prime} \theta:(L, \beta) \Rightarrow\left(L, \beta^{\prime \prime}\right)
$$




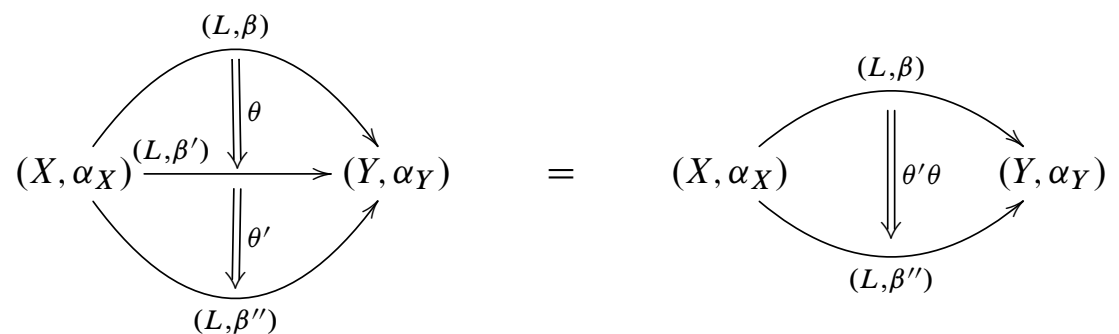

A straightforward calculation implies that

Lemma 4.1. The composition of 1-cells and 2-cells satisfy the axioms of a 2-category.

4.2. Pseudomonoids. A strict 2-monoidal 2-category is a triple $(\mathcal{B}, \bigotimes, \mathbf{I})$ where $\mathcal{B}$ is a 2-category, $\nabla: \mathcal{B} \times \mathcal{B} \rightarrow \mathcal{B}$ is a 2-functor and $\mathbf{I}$ is an object in $\mathcal{B}$, such that $\bigotimes \circ\left(\bigotimes \times I d_{\mathcal{B}}\right)=\bigotimes \circ\left(I d_{\mathcal{B}} \times \bigotimes\right)$ and $\mathbf{I} \otimes X=X \bigotimes \mathbf{I}=X$ for every object in $\mathcal{B}$ (for more details see [5]).

Definition 4.2. Given a strict 2-monoidal 2-category $(\mathcal{B}, \bigotimes, \mathbf{I})$, a pseudomonoid in $\mathcal{B}$ consists of:

- an object $\mathbf{C} \in \mathcal{B}$,

together with:

- a multiplication 1-cell $\mathbf{m}: \mathbf{C} \otimes \mathbf{C} \rightarrow \mathbf{C}$,

- an identity-assigning 1-cell $\mathbb{I}: \mathbf{I} \rightarrow \mathbf{C}$,

together with the following 2-isomorphisms:

- the associator:

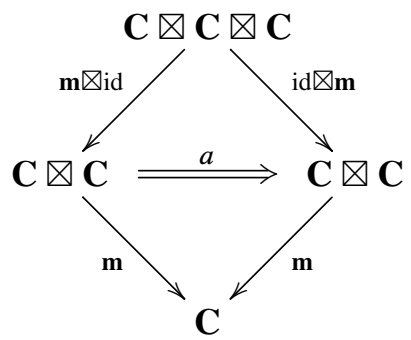

- the left and right unit laws:

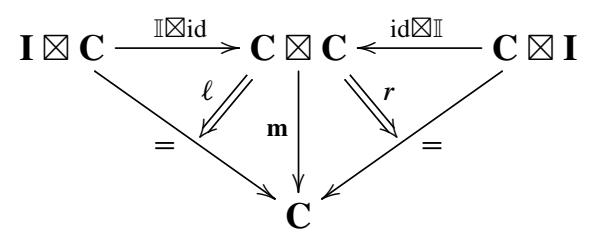


such that the following diagrams commute:

- the pentagon identity for the associator:

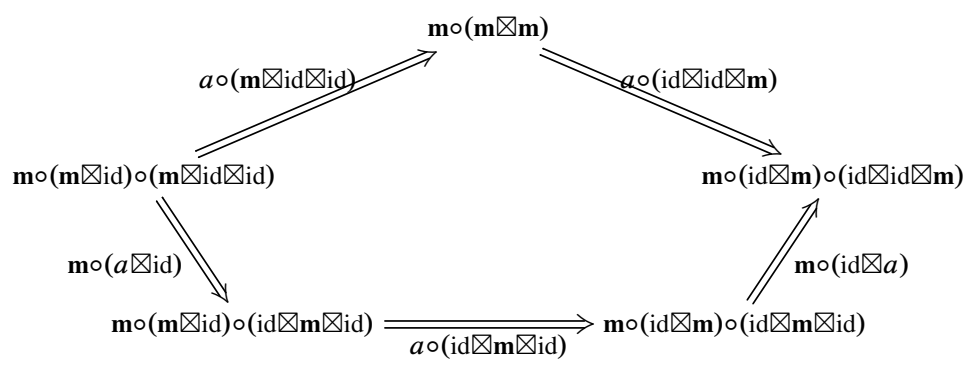

where we use the equalities

$$
\begin{aligned}
& (\mathrm{id} \nabla \mathbf{m}) \circ(\mathbf{m} \nabla \mathrm{id} \nabla \mathrm{id}) \quad=\quad \mathbf{m} \nabla \mathbf{m} \\
& (\mathbf{m} \otimes \mathrm{id}) \circ(\mathrm{id} \otimes \mathrm{id} \otimes \mathbf{m}) \quad=\quad \mathbf{m} \otimes \mathbf{m}
\end{aligned}
$$

in order to compose the upper 1-cells $a \circ(\mathbf{m} \nabla \mathrm{id} \otimes \mathrm{id})$ and $a \circ(\mathrm{id} \otimes \mathrm{id} \nabla \mathbf{m})$, equalities which follow from the fact that $\otimes$ is a 2 -functor.

- the triangle identity for the left and right unit laws:

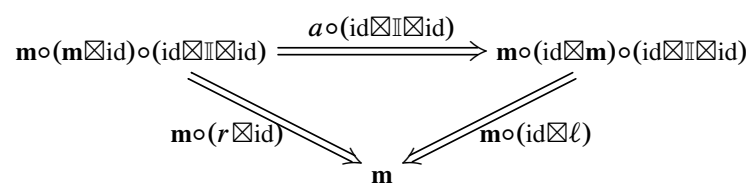

where we use the fact that $\mathbf{C} \otimes \mathbb{I}=\mathbf{C}=\mathbb{I} \otimes \mathbf{C}$ to make sense of the diagonal arrows.

Remark 4.3. The 2-category of $G$-sets with twist has a 2-monoidal structure, where the product of two objects $\left(X, \alpha_{X}\right),\left(Y, \alpha_{Y}\right)$ is given by

$$
\left(X, \alpha_{X}\right) \otimes\left(Y, \alpha_{Y}\right)=\left(X \times Y, \alpha_{X} \otimes \alpha_{Y}\right),
$$

where $X \times Y$ is a $G$-set with the diagonal $G$-action and $\alpha_{X} \otimes \alpha_{Y}:=\pi_{1}^{*}\left(\alpha_{X}\right) \pi_{2}^{*}\left(\alpha_{Y}\right)$. In an analogous way we construct the product $\otimes$ for 1-cells and 2-cells. A unit object is any fixed $G$-set with one element and the constant function 1 for 2-cocycle.

4.3. Pseudomonoids in the 2-category of $G$-sets with twist. We are interested in studying pseudomonoids in the 2-category of $G$-sets with twists, but in order to get normalized cocycles we are forced to consider only pseudomonoids where the identity-assigning 1-cell is strict in the sense that the cochain (the second component of the 1-cell) is trivial, and furthermore, that the unit constraints in diagram (4.2) are identities, namely that the diagram (4.2) commutes. We will call these pseudomonoids with strict unit. 
Proposition 4.4. A pseudomonoid with strict unit in the 2-category of $G$-sets with twists is equivalent to the following data:

- A monoid $(K, m, 1)$, where $K$ is a $G$-set, $m$ is $G$-equivariant and $1 \in K$ is a $G$-invariant element.

- $\alpha \in C^{2,1}(K \rtimes G, \mathbb{T}), \beta \in C^{1,2}(K \rtimes G, \mathbb{T}), \theta \in C^{0,3}(K \rtimes G, \mathbb{T})$ such that $\alpha \oplus \beta \oplus \theta$ is a three cocycle in $\left(\operatorname{Tot}\left(A^{*, *}(K \rtimes G, \mathbb{T})\right), \delta_{G} \oplus \delta_{K}^{(-1)^{p}}\right)$ with $A^{*, *}(K \rtimes G, \mathbb{T})$ the double complex introduced in Definition 2.8.

Proof. A pseudomonoid in the 2-category of $G$-sets is:

i) An object $\mathbf{C}=(K, \alpha)$ where $K$ is a $G$-set and

$$
\alpha: G \times G \times K \rightarrow \mathbb{T}
$$

such that $\alpha$ is normalized in the components of the group $G$ and that $\delta_{G} \alpha=1$.

ii) A multiplication 1-cell

$$
\mathbf{m}=(m, \beta):(K \times K, \alpha \otimes \alpha) \rightarrow(K, \alpha)
$$

such that $m: K \times K \rightarrow K$ is a $G$-equivariant map, and a map

$$
\beta: G \times K \times K \rightarrow \mathbb{T}
$$

satisfying the equation

$$
\delta_{G} \beta=m^{*} \alpha \cdot(\alpha \otimes \alpha)^{-1} .
$$

iii) An identity-assigning 1-cell

$$
\mathbb{I}=\left(\mathbf{1}_{K}, \gamma\right):(\{*\}, 1) \rightarrow(K, \alpha)
$$

where $\mathbf{1}_{K}:\{*\} \rightarrow K$ is a map choosing a $G$-invariant element $1_{K}:=\mathbf{1}_{K}(*)$ in $K$, and $\gamma: G \times\{*\} \rightarrow \mathbb{T}$ is the constant map 1 because we are only considering pseudomonoids with strict unit. The cochain condition on $\gamma$ reads

$$
\left(\delta_{G} \gamma\right)\left[g_{1}\left|g_{2}\right| \mid *\right]=\alpha\left[g_{1}\left|g_{2}\right| \mid 1_{K}\right]
$$

and since $\gamma$ is the constant function it follows that $\alpha\left[g_{1}\left|g_{2}\right| \mid 1_{K}\right]=1$, namely that $\alpha$ is normalized in the $K$ variable.

iv) The left hand side of diagram (4.2) translates to the diagram

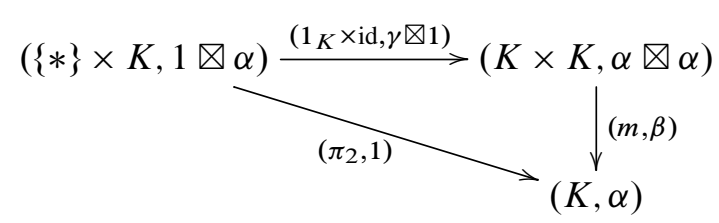


where $\pi_{2}$ is the projection on the second component. The composition of the 1 at the level of the $G$-sets implies the equation

$$
m\left(1_{K}, h\right)=h
$$

for any element $h \in K$. Now, the composition of the 1-cells at the level of the cochains implies the equation

$$
\gamma[g \| *] \cdot \beta\left[g|| 1_{K} \mid h\right]=1
$$

with $g \in G$ and $h \in K$. Since the cochain $\gamma$ is equal to the constant function 1, we have that $\beta\left[g|| 1_{K} \mid h\right]=1$. Applying the same arguments as above we conclude that $\beta\left[g|| h \mid 1_{K}\right]=1$ and therefore the left and right unit laws imply that $\beta$ is normalized on the $K$ components, and moreover that $1_{K}$ is a unit for the multiplication map $m$.

v) Diagram (4.1) translates to the diagram

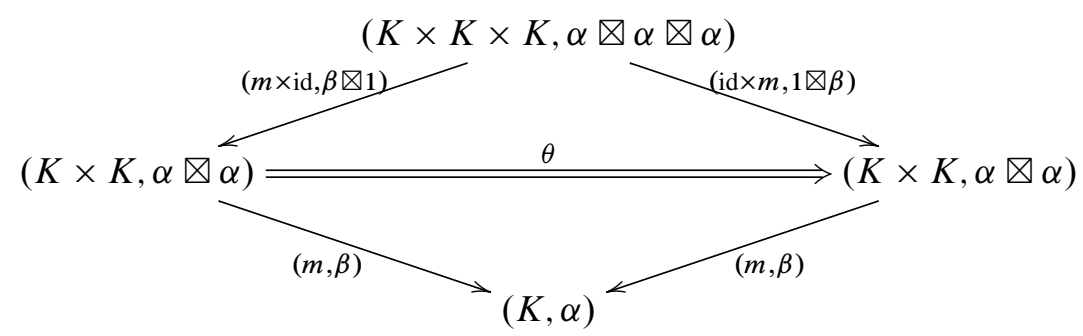

whose commutativity at the level of $G$-sets implies that the multiplication $m$ : $K \times K \rightarrow K$ is associative, and at the level of cochains the diagram implies the equation

$$
\begin{aligned}
& \left(\delta_{G} \theta\right)\left[g \| h_{1}\left|h_{2}\right| h_{3}\right] \\
& \quad=\beta\left[g|| h_{2} \mid h_{3}\right] \beta\left[g|| h_{1} h_{2} \mid h_{3}\right]^{-1} \beta\left[g \| h_{1} \mid h_{2} h_{3}\right] \beta\left[g \| h_{1} \mid h_{2}\right]^{-1} .
\end{aligned}
$$

vi) Diagram (4.3) translates into the equation

$$
\theta\left[k_{1} k_{2}\left|k_{3}\right| k_{4}\right] \theta\left[k_{1}\left|k_{2}\right| k_{3} k_{4}\right]=\theta\left[k_{1}\left|k_{2}\right| k_{3}\right] \theta\left[k_{1}\left|k_{2} k_{3}\right| k_{4}\right] \theta\left[k_{2}\left|k_{3}\right| k_{4}\right]
$$

for all elements $k_{1}, k_{2}, k_{3}, k_{4}$ in $K$.

vii) Diagram (4.4) translates into the equality

$$
\theta\left[k_{1}\left|1_{K}\right| k_{2}\right]=1
$$

for all $k_{1}, k_{2} \in K$, because the unit constraints are trivial. 
From $i$ i) we have that the multiplication map $m: K \times K \rightarrow K$ is a $G$-equivariant map and $v$ ) tells us that the multiplication $m$ is associative. From iii) we know that $K$ is provided with a $G$ invariant element $1_{K}$ and $i v$ ) tells us that this element is a left and right unit for the multiplication $m$. We have then that $\left(K, m, 1_{K}\right)$ is a monoid endowed with a $G$-action compatible with $m$ and $1_{K}$.

Since $K$ is a $G$-equivariant monoid with unit, we can use the notation of Definition 2.4 to see that equation (4.5) can be written as

$$
\delta_{G}(\beta) \delta_{K}(\alpha)=1
$$

equation (4.6) becomes

$$
\delta_{G}(\theta) \delta_{K}(\beta)^{-1}=1
$$

and equation (4.7) becomes

$$
\delta_{K}(\theta)=1
$$

We furthermore have that $\delta_{G} \alpha=1$ by the definition of an object in the category of $G$-sets with twist.

Equation (4.8) implies that $\theta$ is normalized in the variable of the middle; this fact, together with the fact that $\delta_{2} \theta=1$ implies that $\theta$ is normalized in all the variables since

$$
\begin{aligned}
& 1=\delta_{K} \theta\left[1_{K}\left|1_{K}\right| k_{1} \mid k_{2}\right]=\theta\left[1_{K}\left|k_{1}\right| k_{2}\right] \\
& 1=\delta_{K} \theta\left[k_{1}\left|k_{2}\right| 1_{K} \mid 1_{K}\right]=\theta\left[k_{1}\left|k_{2}\right| 1_{K}\right] .
\end{aligned}
$$

From iii) we know that $\alpha$ is normalized in the $K$ coordinate, and from $i v$ ) we know that $\beta$ is normalized in the $K$ coordinates. Therefore the maps $\alpha, \beta$ and $\theta$ are normalized in all variables since their normalization on coordinates of $G$ follow from the definition of the 2-category of $G$-sets with twists.

Summarizing we have that $\alpha \in C^{2,1}(K \rtimes G, \mathbb{T}), \beta \in C^{1,2}(K \rtimes G, \mathbb{T})$ and $\theta \in C^{0,3}(K \rtimes G, \mathbb{T})$ such that $\alpha \oplus \beta \oplus \theta$ is a three cocycle in

$$
\left(\operatorname{Tot}\left(A^{*, *}(K \rtimes G, \mathbb{T})\right), \delta_{G} \oplus \delta_{K}^{(-1)^{p}}\right)
$$

because we have that

$$
\begin{aligned}
\left(\delta_{G} \oplus \delta_{K}^{(-1)^{p}}\right)(\alpha \oplus \beta \oplus \theta) & =\delta_{G}(\alpha) \oplus \delta_{K}(\alpha) \delta_{G}(\beta) \oplus \delta_{K}(\beta)^{-1} \delta_{G}(\theta) \oplus \delta_{K}(\theta) \\
& =1 \oplus 1 \oplus 1 \oplus 1,
\end{aligned}
$$


or in a diagram

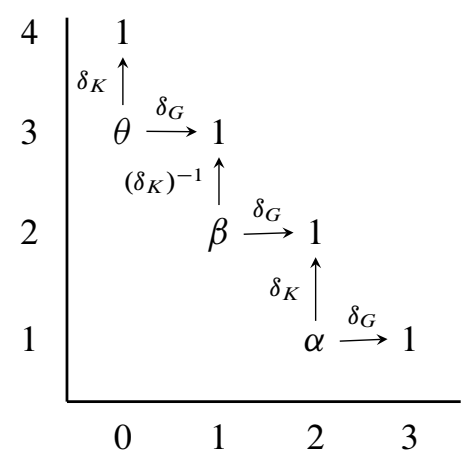

From the construction above, it is easy to see that if we are given the $G$ equivariant monoid $\left(K, m, 1_{K}\right)$ with unit plus the cocycle $\alpha \oplus \beta \oplus \theta$, then we can construct in a unique way a pseudomonoid with strict unit in the 2-category of $G$-sets with twists. This finishes the proof.

Remark 4.5. For a fixed $G$ equivariant monoid $\left(K, m, 1_{K}\right)$, the possible pseudomonoid structures with strict unit in the 2-category of $G$-sets associated over $\left(K, m, 1_{K}\right)$ are classified by elements in

$$
Z^{3}\left(\operatorname{Tot}\left(A^{*, *}(K \rtimes G, \mathbb{T})\right)\right),
$$

namely, 3-cocycles in the total complex of $A^{*, *}(K \rtimes G, \mathbb{T})$.

Definition 4.6. Given pseudomonoids $(\mathbf{C}, \mathbf{m}, \mathbb{I}, a)$ and $\left(\mathbf{C}^{\prime}, \mathbf{m}^{\prime}, \mathbb{I}^{\prime}, a^{\prime}\right)$ in a 2-category $\mathcal{B}$, a morphism

$$
\mathcal{F}:(\mathbf{C}, \mathbf{m}, \mathbb{I}, a) \longrightarrow\left(\mathbf{C}^{\prime}, \mathbf{m}^{\prime}, \mathbb{I}^{\prime}, a^{\prime}\right)
$$

consists of:

- a 1-cell $\mathbb{F}: \mathbf{C} \rightarrow \mathbf{C}^{\prime}$

equipped with:

- a 2-isomorphism

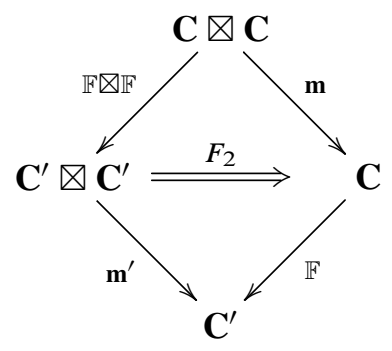


- a 2-isomorphism

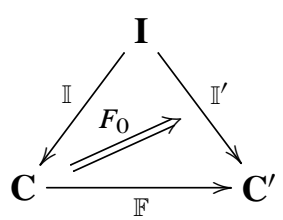

such that diagrams expressing the following laws commute:

- compatibility of $F_{2}$ with the associator:

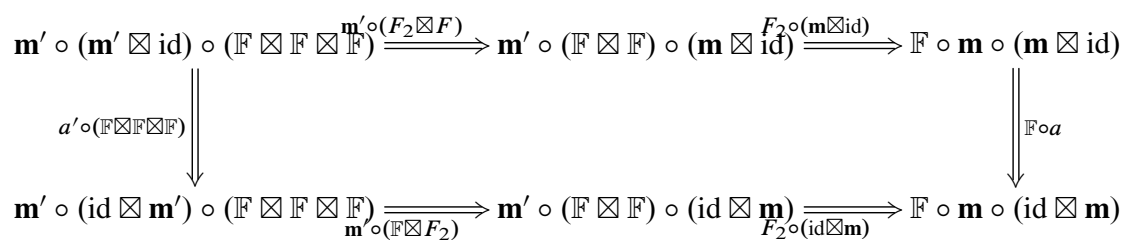

- compatibility of $F_{0}$ with the left unit law:

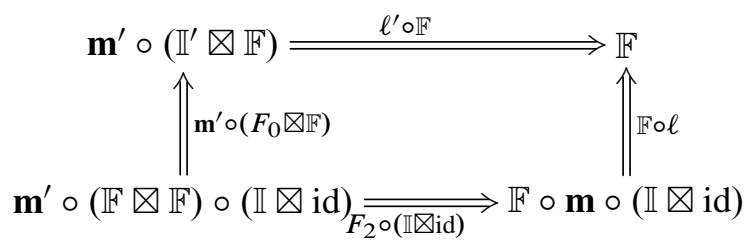

- compatibility of $F_{0}$ with the right unit law:

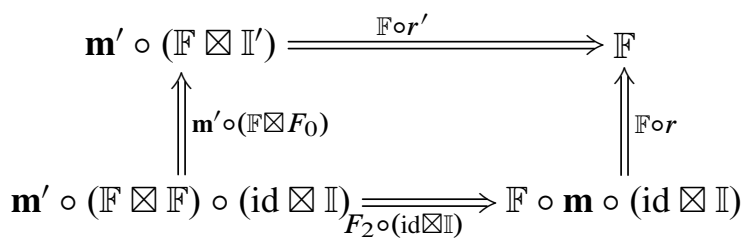

Definition 4.7. Given two pseudomonoids with strict unit $\mathcal{K}=(K, m, 1, \alpha, \beta, \theta)$ and $\mathcal{K}^{\prime}=\left(K^{\prime}, m^{\prime}, 1^{\prime}, \alpha^{\prime}, \beta^{\prime}, \theta^{\prime}\right)$ in the 2-category of $G$-sets with twists, a morphism $\mathcal{F}: \mathcal{K} \rightarrow \mathcal{K}^{\prime}$ is a morphism of pseudomonoids (as in Definition 4.6) such that the 2-isomorphism $F_{0}$ of diagram (4.9) is an identity.

Proposition 4.8. A morphism of pseudomonoids with strict unit in the 2-category of $G$-sets with twists $\mathcal{F}: \mathcal{K} \rightarrow \mathcal{K}^{\prime}$ consists of the triple $\mathcal{F}=(F, \chi, \kappa)$ with

$$
F: K \rightarrow K^{\prime}
$$


a $G$-equivariant morphism of monoids, and cochains $\chi \in C^{1,1}(K \rtimes G, \mathbb{T})$ and $\kappa \in C^{0,2}(K \rtimes G, \mathbb{T})$ such that

$$
\left(\delta_{G} \oplus \delta_{K}^{(-1)^{p}}\right)\left(\chi \oplus \kappa^{-1}\right)=F^{*} \alpha^{\prime} / \alpha \oplus F^{*} \beta^{\prime} / \beta \oplus F^{*} \theta^{\prime} / \theta .
$$

Proof. Following Definition 4.6 a morphism $\mathcal{F}: \mathcal{K} \rightarrow \mathcal{K}^{\prime}$ consists of:

i) A 1-cell $(F, \chi):(K, \alpha) \rightarrow\left(K^{\prime}, \alpha^{\prime}\right)$, i.e. a $G$-equivariant map $F: K \rightarrow K^{\prime}$ and a normalized cochain $\chi \in C_{G}^{1}(K, \mathbb{T})$ such that

$$
\delta_{G} \chi=F^{*} \alpha^{\prime} / \alpha .
$$

ii) A 2-cell $\kappa \in C_{G}^{0}(K \times K, \mathbb{T})$

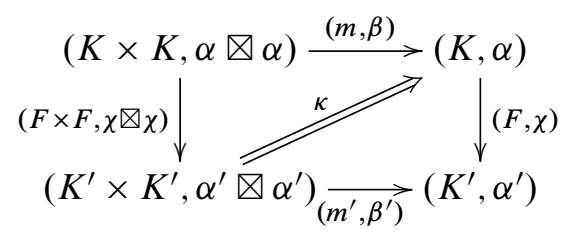

such that

$$
\delta_{G} \kappa=\left(\beta \cdot m^{*} \chi\right) /\left(\chi \otimes \chi \cdot F^{*} \beta^{\prime}\right) .
$$

Note that at the level of the $G$ sets the diagram is commutative, therefore $F$ : $K \rightarrow K^{\prime}$ preserves the multiplication; and since

$$
\delta_{K} \chi=\chi \otimes \chi \cdot\left(m^{*} \chi\right)^{-1},
$$

we can rewrite the equation above as:

$$
\delta_{G} \kappa \cdot \delta_{K} \chi=\beta / F^{*} \beta^{\prime} .
$$

iii) The commutativity of the diagram (4.9)

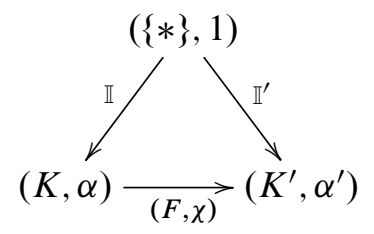

implies that the map $F: K \rightarrow K^{\prime}$ preserves the unit and that $\chi(g, 1)=1$ for any $g \in G$, namely that $\chi$ is normalized in the $K$ variable and therefore we could say that $\chi \in C^{1,1}(K \rtimes G, \mathbb{T})$. 
iv) The commutativity of $\kappa$ with the associator. This implies that $F$ preserves the associativity of the multiplications $m$ and $m^{\prime}$, and that the following equation is satisfied

$\theta^{\prime}\left[F\left(a_{1}\right)\left|F\left(a_{2}\right)\right| F\left(a_{3}\right)\right] \kappa\left[a_{2} \mid a_{3}\right] \kappa\left[a_{1} \mid a_{2} a_{3}\right]=\kappa\left[a_{1} \mid a_{2}\right] \kappa\left[a_{1} a_{2} \mid a_{3}\right] \theta\left[a_{1}\left|a_{2}\right| a_{3}\right]$

for all $a_{1}, a_{2}, a_{3} \in K$. Note that this last equation can be written as

$$
\delta_{K} \kappa=\theta / F^{*} \theta^{\prime} .
$$

v) Compatibility with the left and right units, but as the 2-cells $F_{0}, l, r, l^{\prime}$ and $r^{\prime}$ are identities, then this implies that $\kappa[1 \mid a]=1=\kappa[a \mid 1]$ and therefore we have that $\kappa$ is normalized in the $K$ variables and we can assume that $\kappa \in C^{0,2}(K \rtimes G, \mathbb{T})$.

From the previous arguments it follows that $F: K \rightarrow K^{\prime}$ is a $G$-equivariant morphism of monoids, and moreover, calculating the differential, we have that

$$
\begin{aligned}
\left(\delta_{G} \oplus \delta_{K}^{(-1)^{p}}\right)\left(\chi \oplus \kappa^{-1}\right) & =\delta_{G}(\chi) \oplus \delta_{K}(\chi)^{-1} \delta_{G}(\kappa)^{-1} \oplus \delta_{K}(\kappa)^{-1} \\
& =F^{*} \alpha^{\prime} / \alpha \oplus F^{*} \beta^{\prime} / \beta \oplus F^{*} \theta^{\prime} / \theta,
\end{aligned}
$$

where the second equality follows from equations (4.10), (4.11) and (4.12). In a diagram

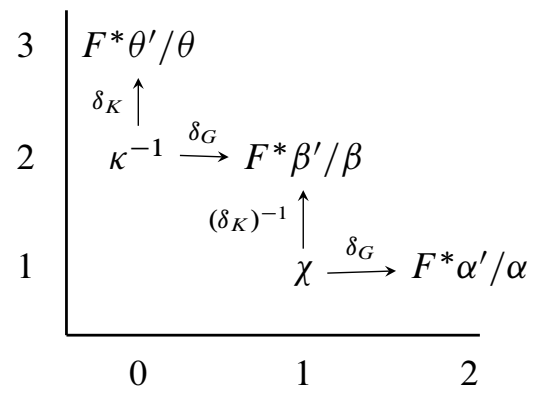

This finishes the proof.

Definition 4.9. Given morphisms $\mathcal{F}=\left(\mathbb{F}, F_{0}, F_{2}\right)$ and $\mathcal{G}=\left(\mathbb{G}, G_{0}, G_{2}\right)$ from $(\mathbf{C}, \mathbf{m}, \mathbb{I}, a)$ to $\left(\mathbf{C}^{\prime}, \mathbf{m}^{\prime}, \mathbb{I}^{\prime}, a^{\prime}\right)$ pseudomonoids in $\mathcal{B}$, a 2-morphism $s: \mathcal{F} \rightarrow \mathcal{G}$ is a 2-cell $s: \mathbb{F} \longrightarrow \mathbb{G}$ in $\mathcal{B}$ such that the following diagrams commute:

- compatibility with $F_{2}$ and $G_{2}$ :

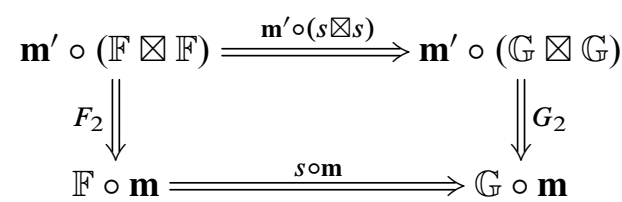


- compatibility with $F_{0}$ and $G_{0}$ :

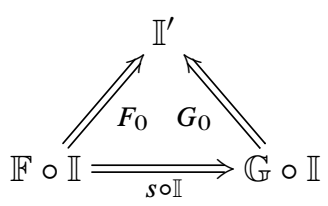

Proposition 4.10. Given morphisms of pseudomonoids with strict unit in the 2-category of $G$-sets with twists $\mathcal{F}=(F, \chi, \kappa)$ and $\mathcal{F}^{\prime}=\left(F, \chi^{\prime}, \kappa^{\prime}\right)$, with $\mathcal{F}, \mathcal{F}^{\prime}$ : $\mathcal{K} \rightarrow \mathcal{K}^{\prime}$, a 2-morphism $\gamma: \mathcal{F} \rightarrow \mathcal{F}^{\prime}$ is a cochain $\gamma: C^{0,1}(K \rtimes G, \mathbb{T})$ such that

$$
\left(\delta_{G} \oplus \delta_{K}^{(-1)^{p}}\right)(\gamma)=\left(\chi^{\prime} / \chi, \kappa / \kappa^{\prime}\right) .
$$

Proof. Let $\gamma:(F, \chi) \rightarrow\left(F, \chi^{\prime}\right)$ be the 2-cell defined by the 2-morphism, then we have that

$$
\delta_{G}(\gamma)=\chi^{\prime} / \chi
$$

Now, by diagram (4.13) we have that

$$
\delta_{K}(\gamma)=\kappa / \kappa^{\prime},
$$

and by diagram (4.14) we have that $\gamma$ is a normalized cochain.

Remark 4.11. Propositions 4.4, 4.8 and 4.10 imply that the relevant information encoded in cochains for the 2-category of pseudomonoids with strict unit of $G$-sets with twists, is given by the cochains of the total complex

$$
\left(\operatorname{Tot}\left(A^{*, *}(K \rtimes G, \mathbb{T})\right), \delta_{G} \oplus \delta_{K}^{(-1)^{p}}\right)
$$

For a fixed $G$-equivariant monoid $\left(K, m, 1_{K}\right)$, Proposition 4.4 tells us that the 3 -cocycles $Z^{3}\left(\operatorname{Tot}^{*}\left(A^{*, *}(K \rtimes G, \mathbb{T})\right)\right)$ are in one to one correspondence with the set of possible pseudomonoid structures with strict unit in the 2-category of $G$-sets with twist over $K$. If we only consider invertible morphisms of pseudomonoids as defined in Proposition 4.8, we may define a groupoid which encodes the equivalence classes of pseudomonoid structures over $K$. Let us be more explicit.

\subsection{Equivalence classes of pseudomonoid structures over a fixed monoid.}

Definition 4.12. Fix a $G$-equivariant monoid $\left(K, m, 1_{K}\right)$. Define the groupoid $\operatorname{Psdmn}^{G}(K)$ whose set of objects is $Z^{3}\left(\operatorname{Tot}^{*}\left(A^{*, *}(K \rtimes G, \mathbb{T})\right)\right)$ and whose morphisms are invertible morphisms of pseudomonoids as defined in Proposition 4.8. The groupoid $\operatorname{Psdmn}^{G}(K)$ encodes the information of all pseudomonoid strucures over $K$ and its coarse moduli space $\left|\operatorname{Psdmn}^{G}(K)\right|$, i.e. the set of equivalence classes defined by the morphisms, is the set of equivalence classes of pseudomonoid structures on $K$. 
Note that a morphism in $\operatorname{Psdmn}^{G}(K)$ consists of a triple $(F, \chi, \kappa)$ where $F: K \rightarrow K$ must be a $G$-equivariant automorphism. If we denote by

$$
\operatorname{Aut}_{G}(K):=\{f \in \operatorname{Aut}(K): f(g k)=g f(k) \text { for all } g \in G\}
$$

then we have that the group $\operatorname{Aut}_{G}(K)$ is isomorphic to the subgroup of $\operatorname{Aut}(K \rtimes G)$ which leaves the $G$ fixed; for a $G$-equivariant automorphism $f \in \operatorname{Aut}_{G}(K)$ we can associate the automorphism $\bar{f} \in \operatorname{Aut}(K \rtimes G)$ by the equation

$$
\bar{f}(k, g):=(f(k), g) .
$$

Since the automorphism $\bar{f}$ leaves $G$ fixed, then the groups of automorphism act on the double complex $A^{*, *}(K \rtimes G, \mathbb{Z})$; we claim

Lemma 4.13. The set of equivalence classes of pseudomonoid structures on $K$ can be described by the quotient

$$
\left|\operatorname{Psdmn}^{G}(K)\right| \cong H^{3}\left(\operatorname{Tot}^{*}\left(A^{*, *}(K \rtimes G, \mathbb{T})\right)\right) / \operatorname{Aut}_{G}(K) .
$$

Proof. We first perform the quotient with the morphisms $(F, \chi, \kappa)$ where $F$ is the identity on $K$; this quotient is precisely $H^{3}\left(\operatorname{Tot}^{*}\left(A^{*, *}(K \rtimes G, \mathbb{T})\right)\right)$. Then we see that elements of $H^{3}\left(\operatorname{Tot}^{*}\left(A^{*, *}(K \rtimes G, \mathbb{T})\right)\right)$ lying on the same orbit of the action of $\operatorname{Aut}_{G}(K)$ define equivalent pseudomonoid structures.

In particular we may conclude that if $H^{3}\left(\operatorname{Tot}^{*}\left(A^{*, *}(K \rtimes G, \mathbb{T})\right)\right)=0$, then all pseudomonoid structures with strict unit in the 2-category of $G$-sets with twist over $K$ are isomorphic to the trivial one. Since we are interested in finding pseudomonoid structures with strict unit in the 2-category of $G$-sets with twist over $K$ non isomorphic to the trivial one, we will calculate the group $H^{3}\left(\operatorname{Tot}^{*}\left(A^{*, *}(K \rtimes G, \mathbb{T})\right)\right)$, the group $\operatorname{Aut}_{G}(K)$ and the set $\left|\operatorname{Psdmn}^{G}(K)\right|$ for some particular examples.

The main tool we will use in order to calculate the group $H^{3}\left(\operatorname{Tot}^{*}\left(A^{*, *}(K \rtimes G\right.\right.$, $\mathbb{T}))$ ) will be the Lyndon-Hochschild-Serre spectral sequence. This spectral sequence can be obtained if the complex $\operatorname{Tot}^{*}\left(A^{*, *}(K \rtimes G, \mathbb{T})\right)$ is filtered by the complexes

$$
F^{n}:=\operatorname{Tot}^{*}\left(A^{* \geq n, *}(K \rtimes G, \mathbb{T})\right)
$$

thus defining a spectral sequence whose second page becomes

$$
\bar{E}_{2}^{p, q}=H^{p}\left(G, H^{q}(K, \mathbb{T})\right)
$$

where $G$ acts on $H^{q}(K, \mathbb{T})$ through the induced action of $G$ on $K$; note in particular that

$$
\bar{E}_{2}^{0, q}=H^{q}(K, \mathbb{T})^{G} \quad \text { and } \quad \bar{E}_{2}^{p, 0}=H^{p}(G, \mathbb{T}) .
$$

On the other hand we have that

$$
\operatorname{Aut}_{G}(K) \cong C_{\mathrm{Aut}(K)}(\rho(G))
$$


where $\rho: G \rightarrow \operatorname{Aut}(K)$ determines the action of $G$, and

$$
C_{\text {Aut }(K)}(\rho(G)):=\{f \in \operatorname{Aut}(K):[f, \rho(g)]=1 \text { for all } g \in G\}
$$

is the centralizer of $\rho(G)$ on $\operatorname{Aut}(K)$, consisting of the automorphisms of $K$ which commute with the $G$-action. In particular when $G=\operatorname{Aut}(K)$ we have that $\operatorname{Aut}_{G}(K)=Z(\operatorname{Aut}(K))$. Let us see some examples:

4.4.1. $K=\mathbb{Z} / p$ for prime $p>2$, and $G=\operatorname{Aut}(\mathbb{Z} / p)=\mathbb{Z} /(p-1)$. Here we have that $\operatorname{Aut}_{G}(K)=Z(\operatorname{Aut}(K)) \cong \mathbb{Z} /(p-1)$ and that

$$
\begin{aligned}
& H^{0}\left(G, H^{3}(K, \mathbb{T})\right)=H^{3}(K, \mathbb{T})^{G} \cong(\mathbb{Z} / p)^{\mathbb{Z} /(p-1)}=0 \\
& H^{1}\left(G, H^{2}(K, \mathbb{T})\right)=\operatorname{Hom}(\mathbb{Z} /(p-1), 0)=0 \\
& H^{2}\left(G, H^{1}(K, \mathbb{T})\right)=H^{2}(\mathbb{Z} /(p-1), \mathbb{Z} / p)=0
\end{aligned}
$$

where the last equality follows from the fact that $H^{n}(G, M)$ is annihilated by $|G|$ for all $n>0$ [6, III.10.2]. In this case $H^{3}\left(\operatorname{Tot}^{*}\left(A^{*, *}(K \rtimes G, \mathbb{T})\right)\right)=0$ and therefore all pseudomonoid structures on $K=\mathbb{Z} / p$ are equivalent to the trivial one. This example could be generalized as follows:

4.4.2. Groups with order relatively prime. Let us recall two facts. First, if $G$ is a finite group of order $m, r$ is a positive integer with $(m, r)=1$ and $A^{r}=0$, then $H^{n}(K, A)=0$ for all $n$ and all subgroups $K$ of $G$, see [13, Proposition 1.3.1]. And second, if $e$ is the exponent of $H^{2}(G, \mathbb{T})$ then $e^{2}$ divides the order of $G$, see [13, Theorem 2.1.5].

Then for the case on which $|G|$ is relatively prime to $|K|$ we obtain that

$$
H^{1}\left(G, H^{2}(K, \mathbb{T})\right)=0 \text { and } H^{2}\left(G, H^{1}(K, \mathbb{T})\right)=0 .
$$

Therefore we have that $H^{3}\left(\operatorname{Tot}^{*}\left(A^{*, *}(K \rtimes G, \mathbb{T})\right)\right) \cong H^{3}(K, \mathbb{T})^{G}$ and

$$
\left|\operatorname{Psdmn}^{G}(K)\right| \cong H^{3}(K, \mathbb{T})^{G} / \operatorname{Aut}_{G}(K) .
$$

4.4.3. The dihedral group $D_{n}$ as a semi-direct product.. The dihedral group is isomorphic to $\mathbb{Z} / n \rtimes \mathbb{Z} / 2$ when $\mathbb{Z} / 2$ acts on $\mathbb{Z} / n$ by multiplication of -1 . Since the induced action of $\mathbb{Z} / 2$ on the cohomology ring $H^{*}(\mathbb{Z} / n, \mathbb{Z}) \cong \mathbb{Z}[x] /\langle n x\rangle$ maps $x \mapsto-x$, we have that $x^{2} \mapsto x^{2}$ and therefore $H^{4}(\mathbb{Z} / n, \mathbb{Z})^{\mathbb{Z} / 2}=\mathbb{Z} / n$ and

$$
H^{2}\left(\mathbb{Z} / 2, H^{2}(\mathbb{Z} / n, \mathbb{Z})\right)=H^{2}(\mathbb{Z} / n, \mathbb{Z})^{\mathbb{Z} / 2}=\left\{\begin{array}{ccc}
\mathbb{Z} / 2 & \text { if } & n \text { is even } \\
0 & \text { if } & n \text { is odd. }
\end{array}\right.
$$


Since

$$
H^{4}\left(D_{n}, \mathbb{Z}\right)=\left\{\begin{array}{cl}
\mathbb{Z} / 2 \oplus \mathbb{Z} / 2 \oplus \mathbb{Z} / n & \text { if } n \text { is even } \\
\mathbb{Z} / 2 \oplus \mathbb{Z} / n & \text { if } n \text { is odd }
\end{array}\right.
$$

we know that

$$
H^{3}\left(\operatorname{Tot}^{*}\left(A^{*, *}(\mathbb{Z} / n \rtimes \mathbb{Z} / 2, \mathbb{T})\right)\right)=\left\{\begin{array}{ccc}
\mathbb{Z} / 2 \oplus \mathbb{Z} / n & \text { if } & n \text { is even } \\
\mathbb{Z} / n & \text { if } & n \text { is odd, }
\end{array}\right.
$$

and therefore

$$
\left|\operatorname{Psdmn}^{\mathbb{Z} / 2}(\mathbb{Z} / n)\right|=\left\{\begin{array}{cc}
(\mathbb{Z} / 2 \oplus \mathbb{Z} / n) / \operatorname{Aut}(\mathbb{Z} / n) & \text { if } n \text { is even } \\
(\mathbb{Z} / n) / \operatorname{Aut}(\mathbb{Z} / n) & \text { if } n \text { is odd. }
\end{array}\right.
$$

because in this case $\operatorname{Aut}_{\mathbb{Z} / 2}(\mathbb{Z} / n)=\operatorname{Aut}(\mathbb{Z} / n)$.

In particular, when $n=4$ we have that $\operatorname{Aut}(\mathbb{Z} / 4)=\mathbb{Z} / 2$. and therefore the action of $\operatorname{Aut}_{\mathbb{Z} / 2}(\mathbb{Z} / 4)$ on $H^{3}\left(\operatorname{Tot}^{*}\left(A^{*, *}(\mathbb{Z} / 4 \rtimes \mathbb{Z} / 2, \mathbb{T})\right)\right)$ is trivial. Hence

$$
\left|\operatorname{Psdmn}^{\mathbb{Z} / 2}(\mathbb{Z} / 4)\right|=\mathbb{Z} / 2 \oplus \mathbb{Z} / 4 \text {. }
$$

4.5. The case of the group acting on itself by conjugation. Perhaps the most known pseudomonoid with strict unit in the 2-category of $G$-sets with twist was introduced by Dijkgraaf, Pasquier and Roche in [7, Section 3.2] while defining the quasi Hopf algebra $D^{w}(G)$ with $w \in Z^{3}(G ; \mathbb{T})$. In the equations (3.2.5) and (3.2.6) of [7] they defined a 3-cocycle $\alpha_{w} \oplus \beta_{w} \oplus \theta_{w} \in Z^{3}\left(\operatorname{Tot}\left(A^{*, *}(G \rtimes G, \mathbb{T})\right)\right)$ by the equations

$$
\begin{aligned}
\alpha_{w}[g|h| \mid x] & :=\frac{w[g|h| x] w\left[g h x h^{-1} g^{-1}|g| h\right]}{w\left[g\left|h x h^{-1}\right| h\right]} \\
\beta_{w}[g|| x \mid y] & :=\frac{w[g|x| y] w\left[g x g^{-1}\left|g y g^{-1}\right| g\right]}{w\left[g x g^{-1}|g| y\right]} \\
\theta_{w}[x|y| z] & :=w[x|y| z],
\end{aligned}
$$

where $\alpha_{w}$ was used to define the algebra law, $\beta_{w}$ to define the coalgebra law, and $\theta_{w}$ encoded the fact that the coproduct is quasicoassociative (to be precise, in order to get exactly the same formulæ as in [7], it is necessary to change $G$ by $G^{o p}$ ).

This quasi Hopf algebra $D^{w}(G)$ is known as the Twisted Drinfeld Double of $G$ twisted by $w$ (cf. [19]). Firstly we claim the following.

Lemma 4.14. The 3-cocycle $\alpha_{w} \oplus \beta_{w} \oplus \theta_{w}$ equals $\tau_{1}^{\vee} w$, the image of $w$ under the restricted shuffle homomorphis $\tau_{1}^{\vee} w$ defined in (3.2). 
Proof. From the following diagrams associated to $\left(\tau_{1}^{\vee} w\right)[g|h| k]$
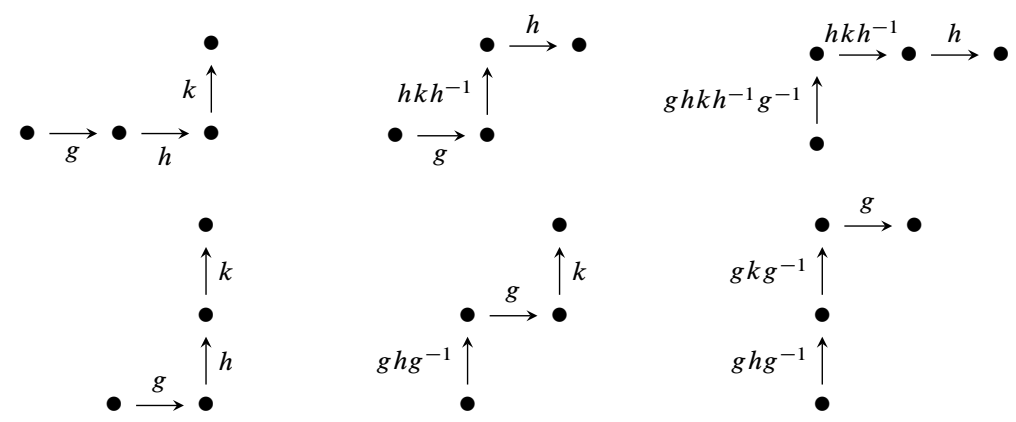

we obtain that $\alpha_{w} \oplus \beta_{w} \oplus \theta_{w}=\tau_{1}^{\vee} w$.

Therefore by Lemma 2.9, Theorem 3.2 and Lemma 3.5 we get

Proposition 4.15. The cohomology class

$$
\left[\alpha_{w} \oplus \beta_{w} \oplus \theta_{w}\right] \oplus[w] \in H^{3}\left(\operatorname{Tot}^{*}\left(A^{* * *}(G \rtimes G, \mathbb{T})\right)\right) \oplus H^{3}(G, \mathbb{T})
$$

is equal to $\mu^{*}[w]$ where $\mu: G \rtimes G \rightarrow G,(a, g) \mapsto a g$ is the multiplication map. Moreover we obtain the isomorphism

$$
\begin{aligned}
H^{3}(G, \mathbb{T}) \oplus H^{3}\left(\operatorname{Tot}^{*}\left(B^{*, *}(G \rtimes G, \mathbb{T})\right)\right) & \rightarrow H^{3}\left(\operatorname{Tot}^{*}\left(A^{*, *}(G \rtimes G, \mathbb{T})\right)\right) \\
{[w] \oplus[x] } & \mapsto\left[\alpha_{w} \oplus \beta_{w} \oplus \theta_{w}\right]+[x] .
\end{aligned}
$$

Now, since $G \rtimes G \cong G \times G$, we have that the Lyndon-Hochschild-Serre spectral sequence collapses at the second page. And since the action of $G$ on $G$ is given by conjugation, then the action of $G$ on $H^{*}(G, \mathbb{T})$ is trivial. Hence we have that $H^{3}\left(\operatorname{Tot}\left(B^{*, *}(G \rtimes G, \mathbb{T})\right)\right)$ sits in the middle of the short exact sequence

$0 \rightarrow H^{2}(G, \operatorname{Hom}(G, \mathbb{T})) \rightarrow H^{3}\left(\operatorname{Tot}\left(B^{*, *}(G \rtimes G, \mathbb{T})\right)\right) \rightarrow \operatorname{Hom}\left(G, H^{2}(G, \mathbb{T})\right) \rightarrow 0$.

Moreover, in the present situation we have $\rho(G)=\operatorname{Inn}(G)$, and therefore

$$
\operatorname{Aut}_{G}(G)=C_{\mathrm{Aut}(G)}(\operatorname{Inn}(G)),
$$

namely the group of automorphisms of $G$ which commute with all inner automorphisms.

With the previous calculations at hand we can calculate the group $H^{3}\left(\operatorname{Tot}\left(B^{*, *}(G \rtimes\right.\right.$ $G, \mathbb{T}))$ ) and $\operatorname{Aut}_{G}(G)$ in some particular examples: 
4.5.1. $G$ simple and nonabelian. When $G$ is simple and nonabelian, its abelianization $G /[G, G]$ is trivial. Therefore $\operatorname{Hom}(G, \mathbb{T})$ and $\operatorname{Hom}\left(G, H^{2}(G, \mathbb{T})\right)$ are trivial and hence

$$
H^{3}\left(\operatorname{Tot}^{*}\left(B^{*, *}(G \rtimes G ; \mathbb{T})\right)\right)=0 .
$$

So we have that

$$
\left|\operatorname{Psdmn}^{G}(G)\right| \cong H^{3}(G, \mathbb{T}) / \operatorname{Aut}_{G}(G) .
$$

When $G$ is the alternating group $A_{n}$ we have that $\operatorname{Aut}_{A_{n}}\left(A_{n}\right)=C_{\mathfrak{S}_{n}}\left(A_{n}\right)=1$ and therefore

$$
\left|\operatorname{Psdmn}^{A_{n}}\left(A_{n}\right)\right| \cong H^{3}\left(A_{n}, \mathbb{T}\right), \text { for } n \neq 6 \text { and } n>4 .
$$

In particular when $n=5$ we have that $H^{3}\left(A_{5}, \mathbb{T}\right)=\mathbb{Z} / 120$ and hence

$$
\left|\operatorname{Psdmn}^{A_{5}}\left(A_{5}\right)\right| \cong \mathbb{Z} / 120 \text {. }
$$

4.5.2. Binary icosahedral group. The binary icosahedral group $\bar{A}_{5}$ is a subgroup of $S U(2)$ that can be obtained as the pullback of the diagram

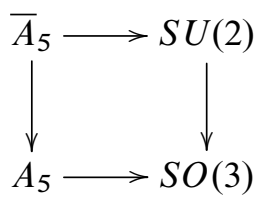

where $A_{5}$ embeds in $S O(3)$ as the group of isometries of an icosahedron. This group satisfies $H_{1}\left(\bar{A}_{5}, \mathbb{Z}\right)=0, H_{2}\left(\bar{A}_{5}, \mathbb{Z}\right)=0$ and $H_{3}\left(\bar{A}_{5}, \mathbb{Z}\right)=\mathbb{Z} / 120$ (see [1, Page 279]), and therefore $H^{1}\left(\bar{A}_{5}, \mathbb{Z}\right)=H^{2}\left(\bar{A}_{5}, \mathbb{Z}\right)=H^{3}\left(\bar{A}_{5}, \mathbb{Z}\right)=0$ and $H^{4}\left(\bar{A}_{5}, \mathbb{Z}\right)=\mathbb{Z} / 120$. Hence $H^{1}\left(\bar{A}_{5}, \mathbb{T}\right)=H^{2}\left(\bar{A}_{5}, \mathbb{T}\right)=0, H^{3}\left(\bar{A}_{5}, \mathbb{T}\right)=\mathbb{Z} / 120$ and $H^{3}\left(\operatorname{Tot}\left(B^{*, *}\left(\bar{A}_{5} \rtimes \bar{A}_{5} ; \mathbb{T}\right)\right)\right)=0$.

In this case $\operatorname{Inn}\left(\bar{A}_{5}\right) \cong A_{5}$ and $\operatorname{Aut}\left(\bar{A}_{5}\right) \cong \mathfrak{S}_{5}$, therefore $\operatorname{Aut}_{\bar{A}_{5}}(G)=$ $C_{\mathfrak{S}_{5}}\left(A_{5}\right)=1$ and

$$
\left|\operatorname{Psdmn}^{A_{5}}\left(A_{5}\right)\right| \cong \mathbb{Z} / 120 .
$$

A similar argument applies to any superperfect group since by definition they are the ones such that $H_{1}(G, \mathbb{Z})=0$ and $H_{2}(G, \mathbb{Z})=0$.

4.5.3. The dihedral group $G=D_{n}$ with $n$ odd. In this case

$$
\operatorname{Hom}\left(D_{n}, \mathbb{T}\right)=\mathbb{Z} / 2, \quad H^{2}\left(D_{n} ; \mathbb{Z} / 2\right)=\mathbb{Z} / 2 \text { and } H^{2}\left(D_{n} ; \mathbb{T}\right)=0,
$$

hence

$$
H^{3}\left(\operatorname{Tot}^{*}\left(B^{*, *}\left(D_{n} \rtimes D_{n} ; \mathbb{T}\right)\right)\right)=H^{2}\left(D_{n}, \operatorname{Hom}\left(D_{n}, \mathbb{T}\right)=\mathbb{Z} / 2 .\right.
$$


Since $H^{3}\left(D_{n} ; \mathbb{T}\right)=\mathbb{Z} / 2 \oplus \mathbb{Z} / n$ we have that the isomorphism classes of pseudomonoid structures coming from the Twisted Drinfeld Double construction are $\mathbb{Z} / 2 \oplus \mathbb{Z} / n$ and that there is an independent pseudomonoid structure which comes from $H^{2}\left(D_{n}, \operatorname{Hom}\left(D_{n} ; \mathbb{T}\right)\right)=\mathbb{Z} / 2$. In this case we have that $\operatorname{Aut}_{D_{n}}\left(D_{n}\right)=$ $Z\left(D_{n}\right)=1$ and therefore

$$
\left|\operatorname{Psdmn}^{D_{n}}\left(D_{n}\right)\right| \cong(\mathbb{Z} / n \oplus \mathbb{Z} / 2 \oplus \mathbb{Z} / 2) .
$$

4.5.4. The symmetric group $G=\mathfrak{S}_{n}$ for $n \geq 4$. From [1, VI-5] we know that

$$
\begin{gathered}
\operatorname{Hom}\left(\mathfrak{S}_{n}, \mathbb{T}\right)=\mathbb{Z} / 2, \quad H^{2}\left(\mathfrak{S}_{n} ; \mathbb{Z} / 2\right)=\mathbb{Z} / 2 \\
H^{2}\left(\mathfrak{S}_{n} ; \mathbb{T}\right)=\mathbb{Z} / 2 \text { and } H^{3}\left(\mathfrak{S}_{n} ; \mathbb{Z} / 2\right)=\mathbb{Z} / 2 \oplus \mathbb{Z} / 2
\end{gathered}
$$

hence we get the exact sequence

$$
\left.0 \rightarrow \mathbb{Z} / 2 \rightarrow H^{3} \operatorname{Tot}^{*}\left(B^{*, *}\left(\mathfrak{S}_{n} \rtimes \mathfrak{S}_{n} ; \mathbb{T}\right)\right)\right) \rightarrow \mathbb{Z} / 2 \rightarrow 0 .
$$

In particular we could say that the nontrivial element in $H^{2}\left(\mathfrak{S}_{n}, \operatorname{Hom}\left(\mathfrak{S}_{n}, \mathbb{T}\right)\right)=$ $\mathbb{Z} / 2$ induces a pseudomonoid structure on $\mathfrak{S}_{n}$ which is not isomorphic to any structure coming from the construction of the Twisted Drinfeld Double. This follows from the fact that for $n \neq 2$ and $n \neq 6$, Aut $\left(\mathfrak{S}_{n}\right)=\operatorname{Inn}\left(\mathfrak{S}_{n}\right)=\mathfrak{S}_{n}$ and therefore Aut $_{\mathfrak{S}_{n}}\left(\mathfrak{S}_{n}\right)=Z\left(\mathfrak{S}_{n}\right)=1$ is the trivial group. Whenever $n=6$ we know that $\operatorname{Out}\left(\mathfrak{S}_{6}\right)=\mathbb{Z} / 2 ;$ nevertheless $\operatorname{Aut}_{\mathfrak{S}_{6}}\left(\mathfrak{S}_{6}\right)=1$. Therefore we have that

$$
\left|\operatorname{Psdmn}{ }^{\mathfrak{S}_{n}}\left(\mathfrak{S}_{n}\right)\right|=H^{3}\left(\mathfrak{S}_{n}, \mathbb{T}\right) \oplus H^{3}\left(\operatorname{Tot}^{*}\left(B^{*, *}\left(\mathfrak{S}_{n} \rtimes \mathfrak{S}_{n} ; \mathbb{T}\right)\right)\right)
$$

with

$$
H^{3}\left(\mathfrak{S}_{n}, \mathbb{T}\right)=\left\{\begin{array}{clc}
\mathbb{Z} / 12 \oplus \mathbb{Z} / 2 & \text { if } \quad n=4,5 \\
\mathbb{Z} / 12 \oplus \mathbb{Z} / 2 \oplus \mathbb{Z} / 2 & \text { if } \quad n \geq 6
\end{array}\right.
$$

4.5.5. $G$ cyclic group. When $G=\mathbb{Z} / n$ we get that

$$
H^{3}\left(\operatorname{Tot}^{*}\left(B^{*, *}(\mathbb{Z} / n \times \mathbb{Z} / n ; \mathbb{T})\right)\right)=H^{2}(\mathbb{Z} / n, \operatorname{Hom}(\mathbb{Z} / n, \mathbb{T}))=\mathbb{Z} / n
$$

and $H^{3}(\mathbb{Z} / n, \mathbb{T})=\mathbb{Z} / n$. The action of $\operatorname{Aut}_{\mathbb{Z} / n}(\mathbb{Z} / n)=\mathbb{Z} / n^{\times}$, which is the multiplicative group of units in $\mathbb{Z} / n$, on $H^{1}(\mathbb{Z} / n, \mathbb{T})=\mathbb{Z} / n$ is given by multiplication and while on $H^{3}(\mathbb{Z} / n, \mathbb{T})=\mathbb{Z} / n$ is given by the square of the multiplication; hence we get that

$$
\left|\operatorname{Psdmn}^{\mathbb{Z} / n}(\mathbb{Z} / n)\right| \cong(\mathbb{Z} / n \oplus \mathbb{Z} / n) / \mathbb{Z} / n^{\times}
$$

where the action is given by

$$
\mathbb{Z} / n^{\times} \times(\mathbb{Z} / n \oplus \mathbb{Z} / n) \rightarrow(\mathbb{Z} / n \oplus \mathbb{Z} / n), \quad(a,(x, y)) \mapsto\left(a x, a^{2} y\right) .
$$

For example when $n=4$ we have that

$$
\left|\operatorname{Psdmn}^{\mathbb{Z} / 4}(\mathbb{Z} / 4)\right| \cong(\mathbb{Z} / 4) /\left(\mathbb{Z} / 4^{\times}\right) \times \mathbb{Z} / 4 .
$$




\section{The monoidal category of equivariant vector bundles on a pesudomonoid}

Let $G$ be a group and $\mathcal{K}=(K, m, 1, \alpha, \beta, \theta)$ a pseudomonoid with strict unit in the 2-category of $G$-sets with twists. We define the category $B u n_{G}(\mathcal{K})$ of $G$-equivariant finite dimensional bundles over $K$ as follows:

An object is a $K$-graded finite dimensional Hilbert space $\mathcal{H}=\bigoplus_{k \in K} \mathcal{H}_{k}$ and a twisted $G$-action

$$
\triangleright: G \rightarrow U(\mathcal{H})
$$

such that

- $\sigma \triangleright \mathcal{H}_{k}=\mathcal{H}_{\sigma \cdot k}$

- $\sigma \triangleright\left(\tau \triangleright h_{k}\right)=\alpha[\sigma|\tau| \mid k](\sigma \tau) \triangleright h_{k}$

- $e \triangleright h=h$

for all $\sigma, \tau \in G, k \in K, h_{k} \in \mathcal{H}_{k}$. Morphisms in the category are linear maps that preserve the grading and the twisted action, i.e., a linear map $f: \mathcal{H} \rightarrow \mathcal{H}^{\prime}$ is a morphism if

- $f\left(\mathcal{H}_{k}\right) \subset \mathcal{H}_{k}^{\prime}$,

- $f(\sigma \triangleright h)=\sigma \triangleright f(h)$

for all $\sigma \in G, k \in K$ and $h \in \mathcal{H}$.

We define a monoidal structure on $\operatorname{Bun}_{G}(\mathcal{K})$ as follows: let $\mathcal{H}$ and $\mathcal{H}^{\prime}$ be objects in $\operatorname{Bun}_{G}(\mathcal{K})$, then the tensor product of Hilbert spaces $\mathcal{H} \otimes \mathcal{H}^{\prime}$ is a $G$-equivariant $K$-bundle with $K$-grading $\left(\mathcal{H} \otimes \mathcal{H}^{\prime}\right)_{k}=\bigoplus_{x, y \in K: x y=k} \mathcal{H}_{x} \otimes \mathcal{H}_{y}^{\prime}$ and twisted $G$-action

$$
\sigma \triangleright\left(h_{x} \otimes h_{y}^{\prime}\right):=\beta[\sigma|| x \mid y]\left(\sigma \triangleright h_{x} \otimes \sigma \triangleright h_{y}^{\prime}\right),
$$

for all $k \in K, \sigma \in G, h_{x} \in \mathcal{H}_{x}$ and $h_{y}^{\prime} \in \mathcal{H}_{y}^{\prime}$.

Now, for $\mathcal{H}, \mathcal{H}^{\prime}$ and $\mathcal{H}^{\prime \prime}$ objects in $B u n_{G}(\mathcal{K})$ the associativity constraint

$$
\Theta:\left(\mathcal{H} \otimes \mathcal{H}^{\prime}\right) \otimes \mathcal{H}^{\prime \prime} \rightarrow \mathcal{H} \otimes\left(\mathcal{H}^{\prime} \otimes \mathcal{H}^{\prime \prime}\right),
$$

for the monoidal structure $\otimes$ is defined by

$$
\Theta\left(\left(h_{x} \otimes h_{y}^{\prime}\right) \otimes h_{z}^{\prime \prime}\right)=\theta[x|y| z]^{-1} h_{x} \otimes\left(h_{y}^{\prime} \otimes h_{z}^{\prime \prime}\right)
$$

for all $x, y, z \in K, h_{x} \in \mathcal{H}_{x}, h_{y}^{\prime} \in \mathcal{H}_{y}^{\prime}$ and $h_{z}^{\prime \prime} \in \mathcal{H}_{z}^{\prime \prime}$.

Finally we define the unit object $\mathbb{C}$ as the one dimensional Hilbert space $\mathbb{C}$ graded only at the unit element $e \in K$, endowed with trivial $G$-action. All in all, we have that

Proposition 5.1. For $\mathcal{K}=(K, m, 1, \alpha, \beta, \theta)$ a pseudomonoid with strict unit in the 2-category of $G$-sets with twists, the triple $\left(B n_{G}(\mathcal{K}), \otimes, \mathbb{C}\right)$, endowed with the tensor product $\otimes$ and the unit element $\mathbb{\mathbb { C }}$ is a monoidal category (or tensor category). 
Remark 5.2. Consider the case of a group $G$ acting on itself by conjugation and the pseudomonoid $\mathcal{K}_{G}^{w}=\left(G, m, 1, \alpha_{w}, \beta_{w}, \theta_{w}\right)$ defined in Section 4.5. In the case that $G$ is finite, the tensor category $B u n_{G}\left(\mathcal{K}_{G}^{w}\right)$ is exactly the category of representations $\operatorname{Rep}\left(D^{w}(G)\right)$ of the Twisted Drinfeld Double $D^{w}(G)$. Note that the quasi-Hopf algebra $D^{w}(G)$ is defined only for $G$ finite, but $B u n_{G}\left(\mathcal{K}_{G}^{w}\right)$ is defined for an arbitrary discrete group. So, the tensor category $B u n_{G}\left(\mathcal{K}_{G}^{w}\right)$ is a generalization of the Twisted Drinfeld Double of a finite group.

5.1. Morphism of pseudomonoids, monoidal functors and natural isomorphisms. A morphism in the 2-category of pseudomonoids in the 2-category $G$-sets with twists induce a monoidal functor between the associated monoidal categories.

Proposition 5.3. Let $\mathcal{F}=(F, \chi, \kappa): \mathcal{K} \rightarrow \mathcal{K}^{\prime}$ be a morphism of pseudomonoids. Then $\mathcal{F}$ induces a monoidal functor from the monoidal categories $\left(B n_{G}(\mathcal{K}), \otimes, \mathbb{C}\right)$ and $\left(\operatorname{Bun}_{G}\left(\mathcal{K}^{\prime}\right), \otimes^{\prime}, \mathbb{C}^{\prime}\right)$.

Proof. Let $\mathcal{F}=(F, \chi, \kappa): \mathcal{K} \rightarrow \mathcal{K}^{\prime}$ be a morphism of pseudomonoids as defined in Proposition 4.8. We define a functor $\mathbf{F}: \operatorname{Bun}_{G}(\mathcal{K}) \rightarrow B u n_{G}\left(\mathcal{K}^{\prime}\right)$ in the following way: for $\mathcal{H}$ an object in $\operatorname{Bun}_{G}(\mathcal{K})$, the $K^{\prime}$-graded Hilbert space $\mathbf{F}(\mathcal{H})$ is the direct sum

$$
\begin{array}{r}
\mathbf{F}(\mathcal{H})_{y}=\bigoplus_{\{x \in K: F(x)=y\}} \mathcal{H}_{x} . \\
\text {. }
\end{array}
$$

The twisted $G$-action on $\mathbf{F}(\mathcal{H})$ is defined as follows: take $h_{y}^{\prime} \in \mathbf{F}(\mathcal{H})_{y}$ defined by the element $h_{y}^{\prime}=h_{x}$ for some vector $h_{x} \in \mathcal{H}_{x}$ with $F(x)=y$. Define the twisted $G$-action $\triangleright^{\prime}$ on $h_{y}^{\prime}$ by

$$
\sigma \triangleright^{\prime} h_{y}^{\prime}:=\chi[\sigma \| x]\left(\sigma \triangleright h_{x}\right) .
$$

The monoidal structure of the functor $\mathbf{F}$ is defined as follows: take $\mathcal{H}, \mathcal{H}^{\prime}$ two

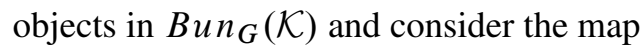

$$
\begin{aligned}
R: \mathbf{F}(\mathcal{H}) \otimes^{\prime} \mathbf{F}\left(\mathcal{H}^{\prime}\right) & \rightarrow \mathbf{F}\left(\mathcal{H} \otimes \mathcal{H}^{\prime}\right) \\
h_{x_{1}} \otimes^{\prime} h_{x_{2}} & \mapsto \kappa\left(x_{1}, x_{2}\right)^{-1} h_{x_{1}} \otimes h_{x_{2}}
\end{aligned}
$$

where $h_{x_{1}} \in \mathcal{H}_{x_{1}}$ and $h_{x_{2}} \in \mathcal{H}_{x_{2}}^{\prime}$ but we see them both as elements in $\mathbf{F}(\mathcal{H})_{F\left(x_{1}\right)}$ and $\mathbf{F}\left(\mathcal{H}^{\prime}\right)_{F\left(x_{2}\right)}$ respectively, and the element $h_{x_{1}} \otimes h_{x_{2}}$ we see it as an element in $\mathbf{F}\left(\mathcal{H} \otimes \mathcal{H}^{\prime}\right)_{F\left(x_{1} x_{2}\right)}$.

Proposition 5.4. Given morphisms of pseudomonoids with strict unit in the 2-category of $G$-sets with twists $\mathcal{F}=(F, \chi, \kappa)$ and $\mathcal{F}^{\prime}=\left(F, \chi^{\prime}, \kappa^{\prime}\right)$, with $\mathcal{F}, \mathcal{F}^{\prime}: \mathcal{K} \rightarrow \mathcal{K}^{\prime}$, a 2-morphism $\gamma: \mathcal{F} \Rightarrow \mathcal{F}^{\prime}$, induces a monoidal natural isomorphism between the monoidal functors $\mathbf{F}$ and $\mathbf{F}^{\prime}$. 
Proof. Using the notation defined above, we define the transformation between $\mathbf{F}$ and $\mathbf{F}^{\prime}$ as follows:

$$
\begin{aligned}
\mathbf{F}(\mathcal{H}) & \rightarrow \mathbf{F}^{\prime}(\mathcal{H}) \\
h_{x} & \mapsto \gamma(x)^{-1} h_{x} .
\end{aligned}
$$

Equations $\delta_{1} \gamma=\chi^{\prime} / \chi$ and $\delta_{2} \gamma=\kappa / \kappa^{\prime}$ shown in the proof of Proposition 4.10 imply that the transformation is natural and monoidal, respectively.

5.2. Automorphisms of pseudomonoids and their action on the monoidal category of equivariant vector bundles. Let us fix $\mathcal{K}=(K, m, 1, \alpha, \beta, \theta)$ a pseudomonoid with strict unit in the 2-category of $G$-sets with twists and let $\left(\operatorname{Bun}_{G}(\mathcal{K}), \otimes, \underline{\mathbb{C}}\right)$ be the monoidal category of $G$-equivariant bundles over $K$.

Take $\mathcal{F}=(F, \chi, \kappa): \mathcal{K} \rightarrow \mathcal{K}$ an invertible morphism of the pseudomonoid $\mathcal{K}$ and note that Proposition 5.3 tells us that the induced monoidal functor

$$
\mathbf{F}:\left(\operatorname{Bun}_{G}(\mathcal{K}), \otimes, \underline{\mathbb{C}}\right) \rightarrow\left(\operatorname{Bun}_{G}(\mathcal{K}), \otimes, \underline{\mathbb{C}}\right)
$$

becomes an automorphism of the monoidal category $\left(B u_{G}(\mathcal{K}), \otimes, \underline{\mathbb{C}}\right)$.

If we denote by

$$
\operatorname{Aut}_{\text {Psmnd }}(\mathcal{K})
$$

the 2-group of automorphisms of the pseudomonoid $\mathcal{K}$, whose morphisms are invertible morphisms $\mathcal{F}: \mathcal{K} \rightarrow \mathcal{K}$ and whose 2-morphisms are the natural transformations between functors $\gamma: \mathcal{F} \Rightarrow \mathcal{F}^{\prime}$, and

$$
\operatorname{Aut}_{\otimes}\left(\operatorname{Bun}_{G}(\mathcal{K})\right)
$$

the 2-group of automorphisms of the monoidal category $\left(B u n_{G}(\mathcal{K}), \otimes, \underline{\mathbb{C}}\right)$, whose morphisms are invertible monoidal functors and whose 2-morphisms are monoidal natural transformations, then we have that Propositions 5.3 and 5.4 imply that there is a 2 -functor

$$
\begin{aligned}
& \operatorname{Aut}_{\text {Psmnd }}(\mathcal{K}) \rightarrow \operatorname{Aut}_{\otimes}\left(\operatorname{Bun}_{G}(\mathcal{K})\right) \\
& \gamma: \mathcal{F} \Rightarrow \mathcal{F}^{\prime} \mapsto \gamma: \mathbf{F} \Rightarrow \mathbf{F}^{\prime}
\end{aligned}
$$

from the 2-group of automorphisms of the pseudomonoid $\mathcal{K}$ to the 2-group of automorpshisms of $\mathrm{Bun}_{G}(\mathcal{K})$.

To understand the previous action in more detail, let us start by studying the category $\operatorname{Aut}_{\text {Psmnd }}(\mathcal{K})$.

An automorphism $\mathcal{F}=(F, \chi, \kappa): \mathcal{K} \rightarrow \mathcal{K}$ consists of a $G$-equivariant automorphism $F \in \operatorname{Aut}_{G}(K)$, together with a degree 2 cochain $\chi \oplus \kappa^{-1}$ in $\operatorname{Tot}^{*}\left(A^{*, *}(K \rtimes G, \mathbb{T})\right)$ such that

$$
\left(\delta_{G} \oplus \delta_{K}^{(-1)^{p}}\right)\left(\chi \oplus \kappa^{-1}\right)=F^{*} \alpha / \alpha \oplus F^{*} \beta / \beta \oplus F^{*} \theta / \theta .
$$


The automorphism $F$ lies on the image of the forgetful functor

$$
\begin{aligned}
\operatorname{Aut}_{\text {Psmnd }}(\mathcal{K}) & \rightarrow \operatorname{Aut}_{G}(K) \\
\mathcal{F}=(F, \chi, \kappa) & \mapsto F ;
\end{aligned}
$$

if and only if the cohomology classes $[\alpha \oplus \beta \oplus \theta]$ and $F^{*}[\alpha \oplus \beta \oplus \theta]$ are equal as cohomology classes in $H^{2}$ ( $\operatorname{Tot}^{*}\left(A^{*, *}(K \rtimes G, \mathbb{T})\right)$ ). If we define

$$
\operatorname{Aut}_{G}(K ;[\alpha \oplus \beta \oplus \theta]):=\left\{F \in \operatorname{Aut}_{G}(K) \mid F^{*}[\alpha \oplus \beta \oplus \theta]=[\alpha \oplus \beta \oplus \theta]\right\}
$$

we have that the 2-group of automorphisms of $\mathcal{K}$ sits in the exact sequence

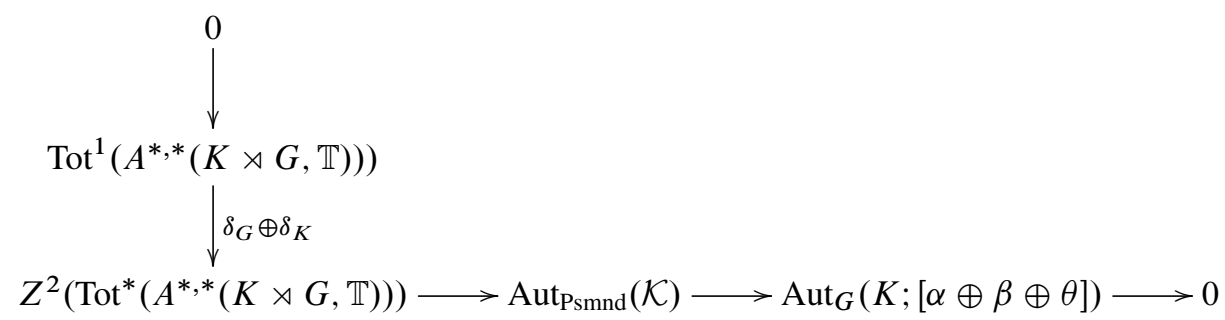

where $Z^{2}\left(\operatorname{Tot}^{*}\left(A^{*, *}(K \rtimes G, \mathbb{T})\right)\right)$ denotes the degree 2-cocycles and $\operatorname{Tot}^{1}\left(A^{*, *}(K \rtimes\right.$ $G, \mathbb{T}))$ parameterizes the 2-morphisms between the morphisms of $Z^{2}\left(\operatorname{Tot}^{*}\left(A^{*, *}(K \rtimes\right.\right.$ $G, \mathbb{T}))$ ).

If we take equivalence classes of automorphisms in $\operatorname{Aut}_{\text {Psmnd }}(\mathcal{K})$ defined by the 2-morphisms, we obtain a group which is usually denoted by

$$
\pi_{1}\left(\operatorname{Aut}_{\mathrm{Psmnd}}(\mathcal{K})\right)
$$

this group sits in the middle of the short exact sequence

$0 \rightarrow H^{2}\left(\operatorname{Tot}^{*}\left(A^{*, *}(K \rtimes G, \mathbb{T})\right)\right) \rightarrow \pi_{1}\left(\operatorname{Aut}_{P_{\text {smnd }}}(\mathcal{K})\right) \rightarrow \operatorname{Aut}_{G}(K ;[\alpha \oplus \beta \oplus \theta]) \rightarrow 0 ;$

and by the Lyndon-Hochschild-Serre spectral sequence we know that there is an exact sequence

$$
\begin{aligned}
0 \rightarrow H^{1}(G, \operatorname{Hom}(K, \mathbb{T})) \rightarrow H^{2}\left(\operatorname{Tot}^{*}\left(A^{*, *}(K \rtimes G, \mathbb{T})\right)\right) \\
\rightarrow H^{2}(K, \mathbb{T})^{G} \stackrel{d_{2}}{\rightarrow} H^{2}(G, \operatorname{Hom}(K, \mathbb{T})),
\end{aligned}
$$

where $d_{2}$ is the differential of the second page.

Furthermore, if we take the group of 2-morphisms of the identity morphism in $\operatorname{Aut}_{\text {Psmnd }}(\mathcal{K})$, we obtain a group which is usually denoted by

$$
\pi_{2}\left(\operatorname{Aut}_{\text {Psmnd }}(\mathcal{K})\right)
$$

and is equal to $H^{1}\left(\operatorname{Tot}\left(A^{*, *}(K \rtimes G, \mathbb{T})\right)\right)=\operatorname{Hom}(K, \mathbb{T})^{G}$. 
5.3. The Grothendieck ring associated to the monoidal category. Consider the isomorphism classes of objects in the monoidal category $\operatorname{Bun}_{G}(\mathcal{K})$. Since the objects could be understood as finite dimensional vector spaces which are $K$-graded endowed with a projective $G$-action, we can add them up and moreover we can multiply them by using the tensor product of the monoidal category. What we obtain is a semi-ring which we can make into a ring by applying the standard Grothendieck construction argument of K-theory. Denoting by Groth $\left(B u n_{G}(\mathcal{K})\right)$ the Grothendieck ring constructed from the monoidal category $B u n_{G}(\mathcal{K})$, we have a functor

$$
\begin{aligned}
\operatorname{Psmnd}_{G} & \rightarrow \text { Rings } \\
\mathcal{K} & \mapsto \operatorname{Groth}\left(\text { Bun }_{G}(\mathcal{K})\right)
\end{aligned}
$$

from the 2-category of pseudomonoids with strict unit in the 2-category $G$-sets with twists, to the category of rings.

The ring Groth $\left(B u n_{G}(\mathcal{K})\right)$ can also be understood as the $\alpha$-twisted $G$-equivariant $\mathrm{K}$-theory of the monoid $K$ where the multiplication is induced by the pushforward $m_{*}$ of the multiplication $m: K \times K \rightarrow K$. This twisted K-theory ring was the main motivation of this work and is the subject of the next section.

In the case in which $G=K$ and $G$ acts on $G$ by the left adjoint action we have seen that any 3-cocycle $w \in Z^{3}(G ; \mathbb{T})$ induces a 3-cocycle $\alpha_{w} \oplus \beta_{w} \oplus$ $\theta_{w} \in Z^{3}\left(\operatorname{Tot}^{*}\left(A^{*, *}(G \rtimes G, \mathbb{T})\right)\right)$ that makes $\mathcal{K}:=\left(G, m, 1, \alpha_{w}, \beta_{w}, \theta_{w}\right)$ into a pseudomonoid with strict unit in the 2-category $G$-sets with twists. In this case the Grothendieck ring Groth $\left(B u n_{G}(\mathcal{K})\right)$ is isomorphic to the Grothendieck ring of representations

$$
\operatorname{Groth}\left(\operatorname{Rep}\left(D^{w}(G)\right)\right)
$$

of the Twisted Drinfeld Double $D^{w}(G)$ of the group $G$ (see [19, Section 3]), which is also isomorphic to the $w$-twisted stringy $\mathrm{K}$-theory

$$
{ }^{w} K_{s t}([G / G])
$$

of the groupoid $[G / G][19$, Prop. 18] c.f. $[2,4,11]$.

5.3.1. Automorphisms. Since the 2 -functor

$$
\operatorname{Aut}_{\text {Psmnd }}(\mathcal{K}) \rightarrow \operatorname{Aut}_{\otimes}\left(\operatorname{Bun}_{G}(\mathcal{K})\right)
$$

induces a homomorphism

$$
\pi_{1}\left(\operatorname{Aut}_{\text {Psmnd }}(\mathcal{K})\right) \rightarrow \pi_{1}\left(\operatorname{Aut}_{\otimes}\left(\operatorname{Bun}_{G}(\mathcal{K})\right)\right),
$$

we have that there is a homomorphism of groups

$$
\pi_{1}\left(\operatorname{Aut}_{\text {Psmnd }}(\mathcal{K})\right) \rightarrow \operatorname{Aut}\left(\operatorname{Groth}\left(B u n_{G}(\mathcal{K})\right)\right)
$$


which composed with the inclusion

$$
H^{2}\left(\operatorname{Tot}\left(A^{*, *}(K \rtimes G, \mathbb{T})\right)\right) \rightarrow \pi_{1}\left(\operatorname{Aut}_{\text {Psmnd }}(\mathcal{K})\right)
$$

defines a homomorphism

$$
H^{2}\left(\operatorname{Tot}\left(A^{*, *}(K \rtimes G, \mathbb{T})\right)\right) \rightarrow \operatorname{Aut}\left(\operatorname{Groth}\left(B u n_{G}(\mathcal{K})\right)\right) .
$$

The previous morphism will be of interest when we compare it with the group of automorphisms of the twisted equivariant K-theory ring in Section 6.7.1.

\section{The fusion product and the twisted $G$-equivariant K-theory ring}

Whenever $X$ is a finite $G$-CW complex with $G$ a finite group, the elements in $H_{G}^{3}(X ; \mathbb{Z})$ classify the isomorphism classes of projective unitary stable and equivariant bundles over $X$, and these bundles provide the required information to define equivariant Fredholm bundles over $X$; the homotopy groups of the space of section of a such bundle is one way to define the twisted $G$-equivariant K-theory groups of $X$ (see [3]). The homotopy classes of automorphisms of a projective unitary stable and equivariant bundle over $X$ are in one to one correspondence with $H_{G}^{2}(X ; \mathbb{Z})$ and this group acts on the twisted $G$-equivariant $\mathrm{K}$-theory groups.

Whenever the space $X$ is a discrete $G$-set, there is an equivalent but easier way to define the twisted $G$-equivariant $\mathrm{K}$-theory groups of $X$. Let us review it.

6.1. Twisted $G$-equivariant K-theory. Take a normalized 2-cocycle $\alpha: G \times G \times$ $X \rightarrow \mathbb{T}$ and define an $\alpha$-twisted $G$-vector bundle over $X$ as a finite dimensional $X$-graded complex vector space $E$, which can alternatively be seen as a finite dimensional complex vector bundle $p: E \rightarrow X$ with finite support, endowed with a $G$ action such that $p$ is $G$ equivariant, the action of $G$ on the fibers is complex linear, and such that the composition of the action on $E$ satisfies the equation

$$
g \cdot(h \cdot z)=\alpha(g, h \| p(z))(g h \cdot z)
$$

for all $z$ in $E$. Two $\alpha$-twisted $G$-vector bundles over $X$ are isomorphic if there exists a $G$ equivariant map $E \rightarrow E^{\prime}$ of complex vector bundles which is an isomorphism of vector bundles.

Definition 6.1. The $\alpha$-twisted $G$-equivariant K-theory of $X$ is the Grothendieck group

$$
\mathbb{K U}_{G}(X ; \alpha)
$$

associated to the semi-group of isomorphism classes of $\alpha$-twisted $G$-vector bundles over $X$ endowed with the direct sum operation. 
If we have a $G$-equivariant map $F: Y \rightarrow X$ then the pullback of bundles induces a group homomorphism

$$
F^{*}: \mathbb{K} \mathbb{U}_{G}(X ; \alpha) \rightarrow \mathbb{K U}_{G}\left(Y ; F^{*} \alpha\right) .
$$

6.1.1. For a normalized cochain $\chi \in C_{G}^{1}(X ; \mathbb{T})$ with $\delta_{G} \chi=\alpha^{\prime} / \alpha$ then there is an induced isomorphism of groups

$$
\bar{\chi}: \mathbb{K} \mathbb{U}_{G}(X ; \alpha) \stackrel{\cong}{\rightarrow} \mathbb{K} \mathbb{U}_{G}\left(X ; \alpha^{\prime}\right)
$$

where $\bar{\chi}(E):=E$ and the $G$-action ${ }^{\prime}$ on $z \in \bar{\chi}(E)$ is given by the equation:

$$
h \bullet^{\prime} z:=\chi[h \| p(z)](h \cdot z) .
$$

Since cohomologous twistings induce isomorphic twisted K-theory groups, we have that $H_{G}^{2}(X ; \mathbb{T})$ classifies the isomorphism classes of twistings for the $G$ equivariant $\mathrm{K}$-theory of $X$. And since the isomorphisms $\bar{\chi}$ and $\overline{\chi \cdot\left(\delta_{G} \gamma\right)}$ are equal, we have that the group $H_{G}^{1}(X ; \mathbb{T})$ acts on the $\alpha$-twisted $G$-equivariant $\mathrm{K}$-theory group $\mathbb{K} \mathbb{U}_{G}(X ; \alpha)$ by automorphisms.

6.2. Pushforward. For a $G$-equivariant map $F: Y \rightarrow X$ and $\alpha \in Z_{G}^{2}(X ; \mathbb{T})$ there is a pushforward map

$$
F_{*}: \mathbb{K} \mathbb{U}_{G}\left(Y ; F^{*} \alpha\right) \rightarrow \mathbb{K} \mathbb{U}_{G}(X ; \alpha)
$$

defined at the level of vector bundles as follows

$$
\begin{array}{r}
\left(F_{*} E\right)_{x}:=\bigoplus E_{y} \\
\{y \in Y \mid F(y)=x\}
\end{array}
$$

where the $G$-action on $F_{*} E$ is the one induced by the $G$-action on $Y$ and the $G$ action on $E$.

6.3. External product. If we consider two $G$-sets with twist $\left(X, \alpha_{X}\right)$ and $\left(Y, \alpha_{Y}\right)$, the external product is the homomorphism

$$
\mathbb{K} \mathbb{U}_{G}\left(X ; \alpha_{X}\right) \times \mathbb{K} \mathbb{U}_{G}\left(Y ; \alpha_{Y}\right) \stackrel{\otimes}{\rightarrow} \mathbb{K} \mathbb{U}_{G}\left(X \times Y ; \pi_{1}^{*} \alpha_{X} \cdot \pi_{2}^{*} \alpha_{Y}\right)
$$

where $(E \otimes F)_{(x, y)}:=E_{x} \otimes F_{y}$ and $\pi_{1}$ and $\pi_{2}$ denote the projections of $X \times Y$ on the first and the second coordinate respectively. 
6.4. Multiplicative structures on Twisted Equivariant K-theory. In the particular case on which the $G$-set $X$ is endowed with the additional structure of a $G$ equivariant multiplication map

$$
m: X \times X \rightarrow X
$$

and moreover that the cohomology class $[\alpha]$ of the twisting is multiplicative i.e. $\pi_{1}^{*}[\alpha] \cdot \pi_{2}^{*}[\alpha]=m^{*}[\alpha]$, then the $\alpha$-twisted $G$-equivariant K-theory group can be endowed with a product structure. This construction could be done for $G$-equivariant $\mathrm{H}$-spaces, but for clarity we will restrict ourselves to the case on which the $G$-set is a $G$-equivariant monoid with unit.

Let $K$ be a $G$-equivariant discrete monoid with unit and denote by $m: K \times$ $K \rightarrow K$ the multiplication of the monoid. Take a twist $\alpha \in Z_{G}^{2}(K ; \mathbb{T})$ that is multiplicative, i.e. that there exist a cochain $\beta \in C_{G}^{1}(K \times K ; \mathbb{T})$ such that

$$
\delta_{G} \beta=\frac{m^{*} \alpha}{\pi_{1}^{*} \alpha \cdot \pi_{2}^{*} \alpha}
$$

or equivalently $\delta_{G} \beta \cdot \delta_{K} \alpha=1$, then we can compose the following morphisms

$$
\begin{aligned}
\mathbb{K} \mathbb{U}_{G}(K ; \alpha) \times \mathbb{K}_{G}(K ; \alpha) \stackrel{\otimes}{\longrightarrow} \mathbb{K} \mathbb{U}_{G}\left(K \times K ; \pi_{1}^{*} \alpha \cdot \pi_{2}^{*} \alpha\right) \\
\stackrel{\bar{\beta}}{\longrightarrow} \mathbb{K} \mathbb{U}_{G}\left(K \times K ; m^{*} \alpha\right) \stackrel{m_{*}}{\longrightarrow} \mathbb{K} \mathbb{U}_{G}(K ; \alpha)
\end{aligned}
$$

thus producing a product structure

$$
\begin{aligned}
\star_{\beta}: \mathbb{K} \mathbb{U}_{G}(K ; \alpha) \times \mathbb{K}_{G}(K ; \alpha) & \rightarrow \mathbb{K} \mathbb{U}_{G}(K ; \alpha) \\
(E, F) & \mapsto m_{*}(\bar{\beta}(\bigotimes(E, F))) .
\end{aligned}
$$

It is a simple calculation to see that the product $\star$ previously defined is associative whenever the cohomology class $[\beta] \in H_{G}^{1}(K \times K ; \mathbb{T})$ satisfies the equation

$$
\delta_{K}[\beta]=1
$$

as a cohomology class in $H_{G}^{1}(K \times K \times K ; \mathbb{T})$. We therefore have that if there exists a cochain $\theta \in C_{G}^{0}(K \times K \times K ; \mathbb{T})$ such that

$$
\delta_{G} \theta=\delta_{K} \beta
$$

then the product $\star$ previously defined endows the group $\mathbb{K} \mathbb{U}_{G}(K ; \alpha)$ with a ring structure. Summarizing:

Proposition 6.2. Consider $\alpha \in Z_{G}^{2}(K ; \mathbb{T})$ and $\beta \in C^{1,2}(K \rtimes G ; \mathbb{T})$ satisfying the equations

$$
\delta_{G} \beta \cdot \delta_{K} \alpha=1, \quad \delta_{2}[\beta]=1 .
$$


Then the group $\mathbb{K} \mathbb{U}_{G}(K ; \alpha)$ endowed with the product structure $\star_{\beta}$ becomes a ring. Let us denote this ring by

$$
\mathbb{K} \mathbb{U}_{G}(K ; \alpha, \beta):=\left(\mathbb{K} \mathbb{U}_{G}(K ; \alpha), \star_{\beta}\right)
$$

and let us call the pair $(\alpha, \beta)$ a multiplicative structure for $K$.

6.4.1. Many of the features of the twisted $G$-equivariant K-theory rings are better understood if we work with the notation introduced in Section 2.

Recall that the double complex $A^{*, *}:=A^{*, *}(K \rtimes G ; \mathbb{T})$ is the subcomplex of $C^{*, *}(K \rtimes G ; \mathbb{T})$ disregarding the 0-th row. Consider the subcomplex $A^{*, *>3}$ of $A^{*, *}$ defined as subcomplex of $C^{*, *}(K \rtimes G ; \mathbb{T})$ where we disregard the first four rows.

The double complex $A^{*, *} / A^{*, *>3}$ consists of the second, third and fourth rows of the double complex $C^{*, *}(K \rtimes G ; \mathbb{T})$, and we have that if $\mathbb{K} \mathbb{U}_{G}(K ; \alpha)$ can be made into a ring is because there exists $\beta$ and $\theta$ such that the cochain $\alpha \oplus \beta \oplus \theta$ becomes a 3-cocycle in the complex $\operatorname{Tot}^{*}\left(A^{*, *} / A^{*, *>3}\right)$ and the 3-cocycle can be seen in a diagram as follows

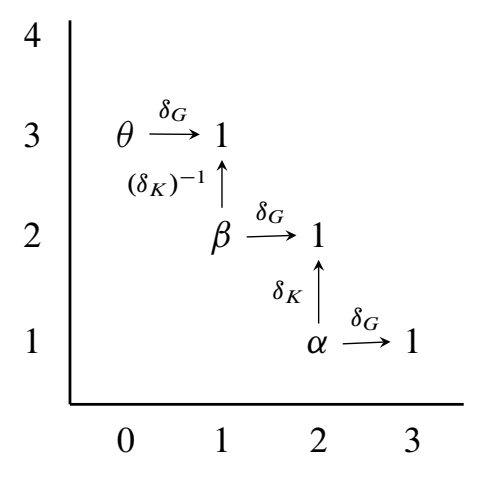

Proposition 6.3. If the cocycle $\alpha \in Z_{G}^{2}(K ; \mathbb{T})$ can be lifted to a 3-cocycle $\alpha \oplus \beta \oplus \theta$ in the complex $\operatorname{Tot}^{*}\left(A^{*, *} / A^{*, *>3}\right)$ then the the group $\mathbb{K} \mathbb{U}_{G}(K ; \alpha)$ can be endowed with the ring structure $\mathbb{K} \mathbb{U}_{G}(K ; \alpha, \beta)$.

6.4.2. Let us see another way to understand the conditions under which the twist $\alpha$ can define a multiplicative structure on $\mathbb{K} \mathbb{U}_{G}(K ; \alpha)$. Consider the filtration of the double complex $A^{*, *}$ given by the subcomplexes $F^{r}:=A^{*, * \geq r}$. The spectral sequence that the filtration defines abuts to the cohomology of the total complex of $A^{*, *}$

$$
E_{\infty}^{*, *} \Rightarrow H^{*}\left(\operatorname{Tot}\left(A^{*, *}\right)\right)
$$

and has for first page

$$
E_{1}^{p, q}=H_{G}^{p}\left(K^{q} ; \mathbb{T}\right)
$$


with differential

$$
d_{1}: E_{1}^{p, q} \rightarrow E_{1}^{p, q+1}, \quad d_{1}[x]:=\left[\left(\delta_{K}\right)^{(-1)^{p}} x\right] .
$$

If we have a twist $\alpha$, its cohomology class $[\alpha]$ is an element in $E_{1}^{2,1}$. The element $d_{1}[\alpha]$ is the first obstruction to lift $\alpha$ to a 3-cocycle in $\operatorname{Tot}^{*}\left(A^{*, *}\right)$, that is, $d_{1}[\alpha]=1$ if and only if there exists $\beta \in C^{1,2}(K \rtimes G ; \mathbb{T})$ such that $\delta_{G} \beta \cdot \delta_{K} \alpha=1$. Note furthermore that

$$
d_{1}[\alpha]=\frac{m^{*}[\alpha]}{\pi_{1}^{*}[\alpha] \cdot \pi_{2}^{*}[\alpha]}
$$

and therefore we recover what we knew, namely that the twist $\alpha$ may induce a product in $\mathbb{K} \mathbb{U}_{G}(K ; \alpha)$ if and only if the cohomology class $[\alpha]$ is multiplicative.

If the cohomology class $[\alpha]$ is multiplicative, then $[\alpha]$ survives to the second page of the spectral sequence with $[\alpha] \in E_{2}^{2,1}$. The second differential applied to $[\alpha]$ is $d_{2}[\alpha]=\left[\left(\delta_{2} \beta\right)^{-1}\right]$

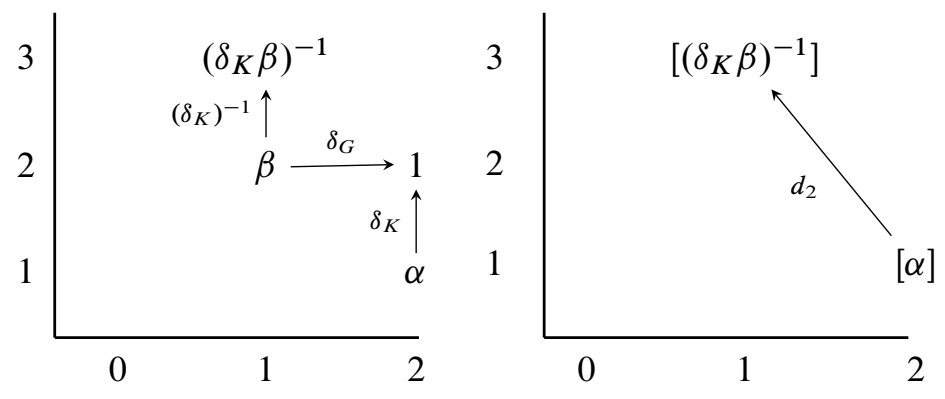

and this is the second obstruction to lift $\alpha$ to a 3-cocycle in $\operatorname{Tot}^{*}\left(A^{*, *}\right)$, i.e. $d_{2}[\alpha]=1$ if and only if there exists $\theta \in C^{0,3}(K \rtimes G, \mathbb{T})$ such that $\delta_{G} \theta\left(\delta_{K} \beta\right)^{-1}=1$. Note that $d_{2}[\alpha]$ measures the obstruction for the multiplication $\star_{\beta}$ in $\mathbb{K} \mathbb{U}_{G}(K ; \alpha)$ to be associative.

We have then that the equations $d_{1}[\alpha]=1=d_{2}[\alpha]$ are the equations that need to be satisfied in order for the group $\mathbb{K} \mathbb{U}_{G}(K ; \alpha)$ to become a ring with respect to the construction provided in this section.

If $[\alpha]$ survives to the third page we have that $d_{3}[\alpha]=\left[\delta_{K} \theta\right]$ and therefore $d_{3}[\alpha]=1$ implies that $\alpha \oplus \beta \oplus \theta$ is a 3-cocycle in $\operatorname{Tot}^{*}\left(A^{*, *}\right)$. So if $[\alpha]$ survives to the fourth page, and hence the page at infinity, then $\mathcal{K}=(K, m, 1, \alpha, \beta, \theta)$ is a pseudomonoid with strict unit in the 2-category of $G$-sets with twists. Summarizing:

Proposition 6.4. Consider $\alpha \in Z_{G}^{2}(K ; \mathbb{T})$, then $\alpha$ can be lifted to a 3-cocycle $\alpha \oplus \beta \oplus \theta$ in $\operatorname{Tot}^{*}\left(A^{*, *} / A^{*, *>3}\right)$ if and only if $d_{1}[\alpha]=1=d_{2}[\alpha]$, and this implies that $\mathbb{K} \mathbb{U}_{G}(K ; \alpha, \beta)$ becomes a ring. If furthermore $d_{3}[\alpha]=1$ then $\alpha \oplus \beta \oplus \theta$ is a 3-cocycle in $\operatorname{Tot}\left(A^{*, *}\right)$ and this implies that $\mathcal{K}=(K, m, 1, \alpha, \beta, \theta)$ is a pseudomonoid with strict unit in the 2-category of $G$-sets with twists, and in this case

$$
\mathbb{K} \mathbb{U}_{G}(K ; \alpha, \beta) \cong \operatorname{Groth}\left(B u n_{G}(\mathcal{K})\right) .
$$


6.5. Isomorphism classes of Multiplicative structures. In this section we want to determine some sufficient conditions under which the twisted $G$-equivariant K-theory rings $\mathbb{K} \mathbb{U}_{G}(K ; \alpha, \beta)$ and $\mathbb{K} \mathbb{U}_{G}\left(K ; \alpha^{\prime}, \beta^{\prime}\right)$ induced by the multiplicative structures $(\alpha, \beta)$ and $\left(\alpha^{\prime}, \beta^{\prime}\right)$ become isomorphic.

Let us consider the double complex $A^{*, *} / A^{*, *>2}$ which consists of the first and the second row of the double complex $C^{*, *}(K \rtimes G, \mathbb{T})$. We claim

Lemma 6.5. Consider $(\alpha, \beta)$ and $\left(\alpha^{\prime}, \beta^{\prime}\right)$ multiplicative structures on $K$. If $\alpha \oplus \beta$ and $\alpha^{\prime} \oplus \beta^{\prime}$ are cohomologous as 3 -cocycles in $\operatorname{Tot}^{*}\left(A^{*, *} / A^{*, *>2}\right)$, then the rings $\mathbb{K} \mathbb{U}_{G}(K ; \alpha, \beta)$ and $\mathbb{K} \mathbb{U}_{G}\left(K ; \alpha^{\prime}, \beta^{\prime}\right)$ are isomorphic.

Proof. If $\alpha \oplus \beta$ and $\alpha^{\prime} \oplus \beta^{\prime}$ are cohomologous as 3-cocycles in $\operatorname{Tot}^{*}\left(A^{*, *} / A^{*, *>2}\right)$, then there exists a 2-cochain $\chi \oplus \kappa^{-1}$ with $\chi \in A^{1,1}$ and $\kappa \in A^{0,2}$ such that

$$
\delta_{G} \oplus \delta_{K}^{(-1)^{p}}\left(\chi \oplus \kappa^{-1}\right)=\alpha^{\prime} / \alpha \oplus \beta^{\prime} / \beta,
$$

namely that $\delta_{G} \chi=\alpha^{\prime} / \alpha$ and $\left(\delta_{K} \chi\right)^{-1}\left(\delta_{G} \kappa\right)^{-1}=\beta^{\prime} / \beta$, or diagramatically

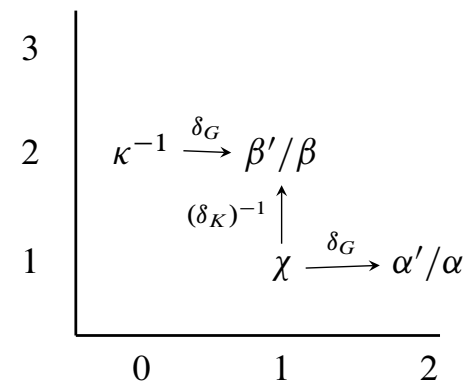

The isomorphism $\bar{\chi}: \mathbb{K} \mathbb{U}_{G}(K ; \alpha) \stackrel{\cong}{\rightarrow} \mathbb{K} \mathbb{U}_{G}\left(K ; \alpha^{\prime}\right)$ induces an isomorphism of rings

$$
\bar{\chi}: \mathbb{K} \mathbb{U}_{G}(K ; \alpha, \beta) \stackrel{\cong}{\rightrightarrows} \mathbb{K} \mathbb{U}_{G}\left(K ; \alpha^{\prime}, \beta^{\prime \prime}\right)
$$

where $\beta^{\prime \prime}:=\beta\left(\delta_{K} \chi\right)^{-1}$. Since $\left(\delta_{K} \chi\right)^{-1}\left(\delta_{G} \kappa\right)^{-1}=\beta^{\prime} / \beta$, we have that $\beta^{\prime \prime}=$ $\beta^{\prime}\left(\delta_{G} \kappa\right)$, therefore the isomorphism $\overline{\beta^{\prime \prime}}$ and $\overline{\beta^{\prime}}$ are equal and we obtain that $\mathbb{K} \mathbb{U}_{G}\left(K ; \alpha^{\prime}, \beta^{\prime \prime}\right) \cong \mathbb{K U}_{G}\left(K ; \alpha^{\prime}, \beta^{\prime}\right)$.

The short exact sequence of complexes

$$
0 \rightarrow A^{*, *>2} / A^{*, *>3} \rightarrow A^{*, *} / A^{*, *>3} \rightarrow A^{*, *} / A^{*, *>2} \rightarrow 0
$$

induces a long exact sequence in cohomology groups

$$
\begin{aligned}
\rightarrow H^{3}\left(\operatorname{Tot}^{*}\left(A^{*, *} / A^{*, *>3}\right)\right) & \rightarrow H^{3}\left(\operatorname{Tot}^{*}\left(A^{*, *} / A^{*, *>2}\right)\right) & \rightarrow H^{4}\left(\operatorname{Tot}^{*}\left(A^{*, *>2} / A^{*, *>3}\right)\right) \\
{[\alpha \oplus \beta \oplus \theta] } & \mapsto \quad[\alpha \oplus \beta] \mapsto & \left.\mapsto\left(\delta_{2} \beta\right)^{-1}\right]
\end{aligned}
$$


and we see that Lemma 6.5 and Proposition 6.4 imply that the subgroup

$$
M S_{G}(K):=\left\{[\alpha \oplus \beta] \in H^{3}\left(\operatorname{Tot}^{*}\left(A^{*, *} / A^{*, *>2}\right)\right) \mid\left[\delta_{K} \beta\right]=1\right\}
$$

is precisely the group of equivalence classes of multiplicative structures associated to the twisted $G$-equivariant K-theory of the monoid $K$. We define

Definition 6.6. The group

$$
M S_{G}(K):=\left\{[\alpha \oplus \beta] \in H^{3}\left(\operatorname{Tot}\left(A^{*, *} / A^{*, *>2}\right)\right) \mid\left[\delta_{2} \beta\right]=1\right\}
$$

will be called the group of multiplicative structures for the $G$-equivariant K-theory of the monoid $K$.

And therefore we have that

Proposition 6.7. The elements of the group $M S_{G}(K)$ are in one to one correspondence with the set of isomorphism classes of ring structures (in the sense of Lemma 6.5) on the twisted $G$-equivariant $K$-theory to the monoid $K$.

In particular we have that there are at most $\#\left(M S_{G}(K)\right)$ of different multiplicative structures in the twisted $G$-equivariant K-theory groups of $K$.

6.6. Automorphisms of the twisted equivariant K-theory ring. From Section 6.1.1 we know that if $\chi \in C_{G}^{1}(K ; \mathbb{T})$ satisfies $\delta_{G} \chi=1$ then the map $\bar{\chi}$ induces an isomorphism of groups

$$
\bar{\chi}: \mathbb{K} \mathbb{U}_{G}(K ; \alpha) \stackrel{\cong}{\rightarrow} \mathbb{K} \mathbb{U}_{G}(K ; \alpha) .
$$

Whenever $(\alpha, \beta)$ is a multiplicative structure, it follows that the map $\bar{\chi}$ induces an isomorphism of rings whenever the homomorphism $\overline{\delta_{K} \chi}$ is the identity map, namely that $\left[\delta_{K} \chi\right]=1$ as a cohomology class in $H_{G}^{1}(K \times K ; \mathbb{T})$. If we define the group of multiplicative elements by

$$
H_{G}^{1}(K ; \mathbb{T})_{\text {mult }}:=\left\{[\chi] \in H_{G}^{1}(K ; \mathbb{T}) \mid \delta_{K}[\chi]=\pi_{1}^{*}[\chi] \cdot \pi_{2}^{*}[\chi] \cdot m^{*}[\chi]^{-1}=1\right\}
$$

we have then that the group of automorphisms of the twisted $G$-equivariant K-theory ring is equal to the multiplicative elements in $H_{G}^{1}(K$; $\mathbb{T})$, i.e.

$$
\operatorname{Aut}\left(\mathbb{K} \mathbb{U}_{G}(K ; \alpha, \beta)\right)=H_{G}^{1}(K ; \mathbb{T})_{m u l t}
$$

Using the spectral sequence of Section 6.4.2 we see that the multiplicative terms appear in the second page of the spectral sequence

$$
H_{G}^{1}(K ; \mathbb{T})_{\mathrm{mult}}=E_{2}^{1,1}
$$


since $d_{1}[\chi]=\left[\delta_{K} \chi\right]^{-1}$ is the obstruction of being multiplicative. If furthermore a multiplicative element satisfies $d_{2}[\chi]=1$, then we have that $[\chi]$ can be lifted to an element $[\chi \oplus \kappa]$ in $H^{2}\left(\operatorname{Tot}\left(A^{*, *}\right)\right)$. This means that we have the exact sequence

$$
0 \rightarrow H^{2}\left(C^{*}(K ; \mathbb{T})^{G}\right) \rightarrow H^{2}\left(\operatorname{Tot}\left(A^{*, *}\right)\right) \rightarrow H_{G}^{1}(K ; \mathbb{T})_{\mathrm{mult}} \stackrel{d_{2}}{\rightarrow} H^{3}\left(C^{*}(K ; \mathbb{T})^{G}\right)
$$

where the $G$-invariant cochains come from the first page of the spectral sequence, i.e. $E_{1}^{0, q}=C^{q}(K ; \mathbb{T})^{G}$, and its cohomology appears in the second page, i.e. $E_{2}^{0, q}=H^{q}\left(C^{*}(K ; \mathbb{T})^{G}, \delta_{K}\right)$.

6.7. Relation between the Grothendieck ring associated to a monoidal category and the twisted equivariant $\mathrm{K}$-theory ring. Let us consider a $G$-equivariant monoid with unit $K$. We have seen that to a pseudomonoid with strict unit in the 2-category of $G$-sets with twist $\mathcal{K}=(K, m, 1, \alpha, \beta, \theta)$ over $K$ we can associate the ring $\operatorname{Groth}\left(B u n_{G}(\mathcal{K})\right)$ of isomorphism classes of objects in the monoidal category $\operatorname{Bun}_{G}(\mathcal{K})$. At the same time, since $\alpha \oplus \beta \oplus \theta \in Z^{3}\left(\operatorname{Tot}^{*}\left(A^{*, *}(K \rtimes G, \mathbb{T})\right)\right)$, then $(\alpha, \beta)$ is a multiplicative structure and we get that

$$
\operatorname{Groth}\left(\operatorname{Bun}_{G}(\mathcal{K})\right) \cong \mathbb{K} \mathbb{U}_{G}(K ; \alpha, \beta)
$$

as rings. We have therefore a canonical map

$$
\begin{aligned}
H^{3}\left(\operatorname{Tot}\left(A^{*, *}\right)\right) & \rightarrow M S_{G}(K) \\
{[\alpha \oplus \beta \oplus \theta] } & \mapsto[\alpha \oplus \beta]
\end{aligned}
$$

from the isomorphism classes of pseudomonoid structures with strict unit of $G$-sets with twist over $K$, to the group of multiplicative structures of the $G$-equivariant twisted K-theory groups of $K$. Let us understand this map in more detail.

Consider the projection homomorphism between the exact sequences of complexes

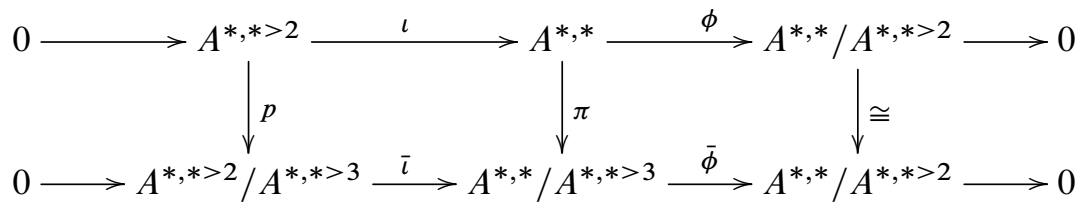

and the homomorphism between the long exact sequences induced

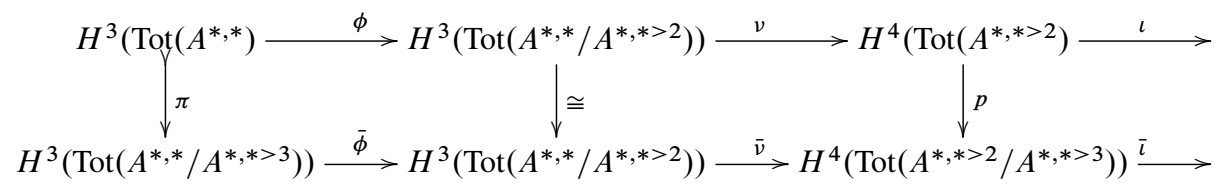

where $v$ and $\bar{v}$ are the connection homomorphisms with $v[\alpha \oplus \beta]=\delta_{2}[\beta]$. 
By definition

$$
M S_{G}(K)=\operatorname{ker}(\bar{v})
$$

and furthermore note that the map $\pi$ is injective since $H^{3}\left(\operatorname{Tot}\left(A^{*, *>3}\right)\right)=0$. Therefore the canonical map defined in (6.2) is precisely the map

$$
H^{3}\left(\operatorname{Tot}\left(A^{*, *}\right)\right) \stackrel{\phi}{\longrightarrow} M S_{G}(K) .
$$

In complete generality it is difficult to give an explicit description of how the kernel and the cokernel of the map $H^{3}\left(\operatorname{Tot}\left(A^{*, *}\right)\right) \stackrel{\phi}{\longrightarrow} M S_{G}(K)$ looks like, but for calculations we can give the following description:

Theorem 6.8. The kernel of the homomorphism $H^{3}\left(\operatorname{Tot}\left(A^{*, *}\right)\right) \stackrel{\phi}{\longrightarrow} M S_{G}(K)$ is isomorphic to

$$
\operatorname{ker}\left(H^{3}\left(\operatorname{Tot}\left(A^{*, *}\right)\right) \stackrel{\phi}{\longrightarrow} M S_{G}(K)\right) \cong \iota\left(H^{3}\left(C^{*}(K, \mathbb{T})^{G}\right)\right)
$$

and the cokernel is isomorphic to

$\operatorname{coker}\left(H^{3}\left(\operatorname{Tot}\left(A^{*, *}\right)\right) \stackrel{\phi}{\longrightarrow} M S_{G}(K)\right) \cong \operatorname{ker}\left(H^{4}\left(C^{*}(K, \mathbb{T})^{G}\right) \rightarrow H^{4}\left(\operatorname{Tot}\left(A^{*, *}\right)\right)\right)$.

Proof. Let us start with the kernel of the map $\phi$. From the long exact sequences of cohomologies defined above we get that

$$
\operatorname{ker}\left(H^{3}\left(\operatorname{Tot}\left(A^{*, *}\right)\right) \stackrel{\phi}{\longrightarrow} M S_{G}(K)\right) \cong \iota\left(H^{3}\left(\operatorname{Tot}\left(A^{*, *>2}\right)\right)\right)
$$

where the group $H^{3}\left(\operatorname{Tot}\left(A^{*, *>2}\right)\right)$ consists of elements in $C^{0,3}$ that are closed under the differentials $\delta_{G}$ and $\delta_{K}$ and therefore

$$
H^{3}\left(\operatorname{Tot}\left(A^{*, *>2}\right)\right)=Z^{3}(K, \mathbb{T})^{G} .
$$

Now, since the elements $\delta_{K}\left(C^{2}(K, \mathbb{T})^{G}\right)$ are all zero in $H^{3}\left(\operatorname{Tot}\left(A^{*, *}\right)\right)$ we have that

$$
\iota\left(Z^{3}(K, \mathbb{T})^{G}\right)=\iota\left(H^{3}\left(C^{*}(K, \mathbb{T})^{G}\right)\right)
$$

and therefore

$$
\operatorname{ker}\left(H^{3}\left(\operatorname{Tot}\left(A^{*, *}\right)\right) \stackrel{\phi}{\longrightarrow} M S_{G}(K)\right) \cong \iota\left(H^{3}\left(C^{*}(K, \mathbb{T})^{G}\right)\right)
$$

For the cokernel we have that the long exact sequence in cohomologies defined above implies that

$$
\operatorname{coker}\left(H^{3}\left(\operatorname{Tot}\left(A^{*, *}\right)\right) \stackrel{\phi}{\longrightarrow} M S_{G}(K)\right) \cong H^{4}\left(\operatorname{Tot}\left(A^{*, *>2}\right)\right) \cap \operatorname{ker}(p) \cap \operatorname{ker}(\iota) .
$$


Now, the projection map

$$
p: H^{4}\left(\operatorname{Tot}\left(A^{*, *>2}\right)\right) \rightarrow H^{4}\left(\operatorname{Tot}\left(A^{*, *>2} / A^{*, *>3}\right)\right)=H_{G}^{1}\left(K^{3}, \mathbb{T}\right)
$$

has for kernel the elements in the fourth cohomology of the $G$-invariant $K$-chains

$$
\operatorname{ker}(p)=H^{4}\left(C^{*}(K, \mathbb{T})^{G}\right),
$$

and this follows from the spectral sequence that converges $H^{*}\left(\operatorname{Tot}\left(A^{*, *>2}\right)\right)$ associated to the filtration $\operatorname{Tot}\left(A^{*>q, *}\right)$. Since the natural homomorphism

$$
H^{4}\left(C^{*}(K, \mathbb{T})^{G}\right) \rightarrow H^{4}\left(\operatorname{Tot}\left(A^{*, *}\right)\right)
$$

coincides with the map $\iota$, we have the desired isomorphism

$\operatorname{coker}\left(H^{3}\left(\operatorname{Tot}\left(A^{*, *}\right)\right) \stackrel{\phi}{\longrightarrow} M S_{G}(K)\right) \cong \operatorname{ker}\left(H^{4}\left(C^{*}(K, \mathbb{T})^{G}\right) \rightarrow H^{4}\left(\operatorname{Tot}\left(A^{*, *}\right)\right)\right)$.

For calculation purposes let us understand the kernel and the cokernel of the map $\phi$ from the point of view of the spectral sequence of Section 6.4.2.

Proposition 6.9. Consider the filtration of the complex $\operatorname{Tot}\left(A^{*, *}\right)$ defined by the subcomplexes $\operatorname{Tot}\left(A^{*, *>q}\right)$ and consider the spectral sequence that it defines which converges to $H^{*}\left(\operatorname{Tot}\left(A^{*, *}\right)\right.$. Then we have the isomorphisms

$$
\begin{aligned}
& \operatorname{ker}\left(H^{3}\left(\operatorname{Tot}\left(A^{*, *}\right)\right) \stackrel{\phi}{\longrightarrow} M S_{G}(K)\right) \cong E_{4}^{0,3} \\
& \operatorname{coker}\left(H^{3}\left(\operatorname{Tot}\left(A^{*, *}\right)\right) \stackrel{\phi}{\longrightarrow} M S_{G}(K)\right) \cong d_{3}\left(E_{3}^{2,1}\right)+d_{2}\left(E_{2}^{1,2}\right) .
\end{aligned}
$$

In the particular case on which the spectral sequence collapses at the second page we conclude that

$$
0 \rightarrow H^{3}\left(C^{*}(K, \mathbb{T})^{G}\right) \rightarrow H^{3}\left(\operatorname{Tot}\left(A^{*, *}\right)\right) \stackrel{\phi}{\longrightarrow} M S_{G}(K) \rightarrow 0 .
$$

Proof. The first page of the spectral sequence associated to the filtration $\operatorname{Tot}\left(A^{*, q>*}\right)$ is given by $E_{1}^{0, q}=C^{q}(K, \mathbb{T})^{G}$ and $E_{1}^{p, q}=H_{G}^{p}\left(K^{q}, \mathbb{T}\right)$ whenever $q>0$, and therefore one has that the second page is given by

$$
E_{2}^{0, q}=H^{q}\left(C^{*}(K, \mathbb{T})^{G}\right)
$$

and for $p>0$ and $q>0$

$$
E_{2}^{p, q}=\frac{\operatorname{ker}\left(H_{G}^{p}\left(K^{q}, \mathbb{T}\right) \stackrel{\delta_{K}}{\rightarrow} H_{G}^{p}\left(K^{q+1}, \mathbb{T}\right)\right)}{\operatorname{im}\left(H_{G}^{p}\left(K^{q-1}, \mathbb{T}\right) \stackrel{\delta_{K}}{\rightarrow} H_{G}^{p}\left(K^{q}, \mathbb{T}\right)\right)} .
$$


The map

$$
H^{4}\left(C^{*}(K, \mathbb{T})^{G}\right) \rightarrow H^{4}\left(\operatorname{Tot}\left(A^{*, *}\right)\right)
$$

coincides with the standard map $E_{2}^{0,4} \rightarrow H^{4}\left(\operatorname{Tot}\left(A^{*, *}\right)\right)$ and its kernel consists of the images of $E_{2}^{1,2}$ and $E_{3}^{2,1}$ under the differentials $d_{2}$ and $d_{3}$ respectively, since we know that the map

$$
E_{4}^{0,4} \hookrightarrow H^{4}\left(\operatorname{Tot}\left(A^{*, *}\right)\right)
$$

is injective. Therefore we have that

$$
d_{2}\left(E_{2}^{1,2}\right) \subset \operatorname{ker}\left(H^{4}\left(C^{*}(K, \mathbb{T})^{G}\right) \rightarrow H^{4}\left(\operatorname{Tot}\left(A^{*, *}\right)\right)\right)
$$

and the above inclusion is an equality whenever $d_{3}\left(E_{3}^{2,1}\right)=0$. In the case that $d_{3}\left(E_{3}^{2,1}\right) \neq 0$ we could abuse of the notation and say that

$$
d_{3}\left(E_{3}^{2,1}\right)+d_{2}\left(E_{2}^{1,2}\right)=\operatorname{ker}\left(H^{4}\left(C^{*}(K, \mathbb{T})^{G}\right) \rightarrow H^{4}\left(\operatorname{Tot}\left(A^{*, *}\right)\right)\right) .
$$

A similar argument could be used to calculate $\iota\left(H^{3}\left(C^{*}(K, \mathbb{T})^{G}\right)\right)$. Since $E_{2}^{0,3}=H^{3}\left(C^{*}(K, \mathbb{T})^{G}\right)$ we have that its image $\iota\left(E_{2}^{0,3}\right) \subset H^{3}\left(\operatorname{Tot}\left(A^{*, *}\right)\right)$ is equal to the image of the canonical map

$$
E_{2}^{0,3} \rightarrow H^{3}\left(\operatorname{Tot}\left(A^{*, *}\right)\right)
$$

Since the image is isomorphic to the group to which it converges, in this case $E_{4}^{0,4}$, then we can conclude that $\iota\left(H^{3}\left(C^{*}(K, \mathbb{T})^{G}\right)\right) \cong E_{4}^{0,3}$ and therefore

$$
\operatorname{ker}\left(H^{3}\left(\operatorname{Tot}\left(A^{*, *}\right)\right) \stackrel{\phi}{\longrightarrow} M S_{G}(K)\right) \cong E_{4}^{0,3}
$$

Finally, whenever the spectral sequence collapses at the second page we have that $d_{2}=0=d_{3}$ and therefore $\phi$ is surjective. Since we have in this case we have that $E_{4}^{0,3}=E_{2}^{0,3}=H^{3}\left(C^{*}(K, \mathbb{T})^{G}\right)$, the proposition follows.

From Theorem 6.8 we can deduce two things.

- If $\mathcal{K}=(K, m, 1, \alpha, \beta, \theta)$ is a pseudomonoid with strict unit in the 2-category of $G$-sets with twists such that $[\alpha \oplus \beta \oplus \theta]$ lies in the image of $\iota$, then the Grothendieck ring $\operatorname{Groth}\left(B u n_{G}(\mathcal{K})\right)$ is isomorphic to the untwisted $\operatorname{ring} \mathbb{K U}_{G}(K)$.

- Multiplicative structures $\left(\alpha^{\prime}, \beta^{\prime}\right)$ in $M S_{G}(K)$ such that $\alpha^{\prime} \oplus \beta^{\prime} \oplus \theta^{\prime}$ belongs to $Z^{3}\left(\operatorname{Tot}\left(A^{*, *} / A^{*, *>3}\right)\right)$ and $\delta_{K} \theta^{\prime} \neq 0$, define ring structures $\mathbb{K} \mathbb{U}_{G}\left(K ; \alpha^{\prime}, \beta^{\prime}\right)$ which cannot be obtained as the Grothendieck ring $\operatorname{Groth}\left(B u n_{G}(\mathcal{K})\right)$ for any pseudomonoid $\mathcal{K}$ with strict unit in the 2-category of $G$-sets with twists. 
6.7.1. Relation between the automorphisms. We have seen that the isomorphism classes of automorphisms of $\mathcal{K}$ that leave the monoid $K$ fixed is isomorphic to the group $H^{2}\left(\operatorname{Tot}\left(A^{*, *}(K \rtimes G, \mathbb{T})\right)\right)$. Since the automorphism group of $\mathbb{K} \mathbb{U}_{G}(K ; \alpha, \beta)$ is $H_{G}^{1}(K ; \mathbb{T})_{\text {mult }}$ we have that there is an induced map

$$
H^{2}\left(\operatorname{Tot}\left(A^{*, *}\right)\right) \rightarrow H_{G}^{1}(K ; \mathbb{T})_{\text {mult }}
$$

which matches the homomorphism that appears in the exact sequence (6.1)

$$
0 \rightarrow H^{2}\left(C^{*}(K ; \mathbb{T})^{G}\right) \rightarrow H^{2}\left(\operatorname{Tot}\left(A^{*, *}\right)\right) \rightarrow H_{G}^{1}(K ; \mathbb{T})_{\text {mult }} \stackrel{d_{2}}{\rightarrow} H^{3}\left(C^{*}(K ; \mathbb{T})^{G}\right) .
$$

Note that in the case that the spectral sequence collapses at the second page we get the short exact sequence

$$
0 \rightarrow H^{2}\left(C^{*}(K ; \mathbb{T})^{G}\right) \rightarrow H^{2}\left(\operatorname{Tot}\left(A^{*, *}\right)\right) \rightarrow H_{G}^{1}(K ; \mathbb{T})_{\mathrm{mult}} \rightarrow 0 .
$$

A more elaborate analysis of the homomorphisms

$$
H^{3}\left(\operatorname{Tot}\left(A^{*, *}\right)\right) \stackrel{\phi}{\rightarrow} M S_{G}(K) \text { and } H^{2}\left(\operatorname{Tot}\left(A^{*, *}\right)\right) \rightarrow H_{G}^{1}(K ; \mathbb{T})_{\text {mult }}
$$

will depend on the choice of the group $G$ and of the $G$-equivariant monoid $K$. In the next chapter we will calculate explicitly the previous homomorphisms for several examples, and from them we will deduce interesting information with regard to the twisted equivariant K-theory rings.

\section{Examples}

The main objective of this section is to use Proposition 6.9 to calculate the kernel and cokernel of the homomorphism

$$
H^{3}\left(\operatorname{Tot}\left(A^{*, *}\right)\right) \stackrel{\phi}{\rightarrow} M S_{G}(K)
$$

for different choices of $G$ and $K$, in order to show the different twisted $G$-equivariant $\mathrm{K}$-theory rings over $K$ that can appear.

7.1. Trivial action of $G$ on $K$. In this case we have that the spectral sequence defined in Proposition 6.9 collapses at the second page and moreover we have that $C^{*}(K, \mathbb{T})=C^{*}(K, \mathbb{T})^{G}$. Therefore we obtain the short exact sequence

$$
0 \rightarrow H^{3}(K, \mathbb{T}) \rightarrow H^{3}\left(\operatorname{Tot}\left(A^{*, *}\right)\right) \stackrel{\phi}{\rightarrow} M S_{G}(K) \rightarrow 0,
$$

thus implying that

$$
M S_{G}(K) \cong H^{3}\left(\operatorname{Tot}^{*}\left(B^{*, *}(K \times G, \mathbb{T})\right)\right),
$$


and moreover that all multiplicative structures for the $G$-equivariant K-theory of $K$ can be obtained from the ring structures defined by the Grothendieck rings of the monoidal categories $\mathrm{Bun}_{G}(\mathcal{K})$. We also obtain the short exact sequence

$$
0 \rightarrow H^{2}(K, \mathbb{T}) \rightarrow H^{2}\left(\operatorname{Tot}\left(A^{*, *}\right)\right) \rightarrow \operatorname{Hom}(K, \operatorname{Hom}(G, \mathbb{T})) \rightarrow 0
$$

where in this case $H_{G}^{1}(K, \mathbb{T})_{m u l t}=\operatorname{Hom}(K, \operatorname{Hom}(G, \mathbb{T}))$.

Furthermore, if $[\theta] \in H^{3}(K, \mathbb{T})$ is non trivial then we can define a nontrivial pseudomoinoid with strict unit in the 2-category of $G$-sets with twist $\mathcal{K}=(K, m, 1,0,0, \theta)$ with $[0 \oplus 0 \oplus \theta]$ non-zero in $H^{3}\left(\operatorname{Tot}\left(A^{*, *}\right)\right)$, such that

$$
\operatorname{Groth}\left(B u n_{G}(\mathcal{K})\right) \cong R(G) \otimes_{\mathbb{Z}} \mathbb{Z}[K]
$$

where $R(G)$ is the Grothendieck ring of finite dimensional complex representations of $G$ and $\mathbb{Z}[K]$ is the group ring of $K$, since we know that $R(G) \otimes_{\mathbb{Z}} \mathbb{Z}[K]$ is isomorphic to the non-twisted ring structure on $\mathbb{K} \mathbb{U}_{G}(K)$.

7.1.1. $G=\mathbb{Z} / n$ and $K=\mathbb{Z} / m$. In this case we have that $H^{3}(K, \mathbb{T})=\mathbb{Z} / m$ and

$$
H^{3}\left(\operatorname{Tot}\left(A^{*, *}\right)\right)=\mathbb{Z} / m \oplus \mathbb{Z} /(n, m)
$$

where $(n, m)$ is the greatest common divisor of the pair $n, m$. Therefore $M S_{G}(K)=$ $\mathbb{Z} /(n, m)$ and we have that all non trivial multiplicative structures come from the group

$$
\mathbb{Z} /(n, m)=H^{3}\left(\operatorname{Tot}\left(B^{*, *}\right)\right) \subset H^{3}\left(\operatorname{Tot}\left(A^{*, *}\right)\right) .
$$

7.2. Adjoint action of $G$ on itself. From Lemma 3.5 we know that in this case we have the split short exact sequence

$$
\left.0 \longrightarrow H^{*}\left(\operatorname{Tot}\left(B^{*, *}\right)\right) \longrightarrow H^{*}\left(\operatorname{Tot}\left(A^{*, *}\right)\right) \stackrel{\mu^{*}}{\longrightarrow} H^{*}(G, \mathbb{T})\right) \longrightarrow 0
$$

induced by the short exact sequence of complexes

$$
0 \rightarrow \operatorname{Tot}\left(B^{*, *}\right) \subset \operatorname{Tot}\left(A^{*, *}\right) \rightarrow C^{*>0}(G, \mathbb{T}) \rightarrow 0 .
$$

Since we have the inclusion $H^{*}\left(\operatorname{Tot}\left(B^{*, *}\right)\right) \subset H^{*}\left(\operatorname{Tot}\left(A^{*, *}\right)\right)$, we can deduce that the groups $E_{2}^{*, 0}$ of the 0-th column of the second page of the spectral sequence converging to $H^{*}\left(\operatorname{Tot}\left(A^{*, *}\right)\right)$ defined in Proposition 6.9, are unaffected by the differentials $d_{i}$ for $i>1$; this follows from the injectivity between the spectral sequences associated to the filtrations $B^{*, *>q}$ and $A^{*, *>q}$ of $\operatorname{Tot}\left(B^{*, *}\right)$ and $\operatorname{Tot}\left(A^{*, *}\right)$ respectively.

Therefore we have that $E_{4}^{0,3}=E_{2}^{0,3}=H^{3}\left(C^{*}(G, \mathbb{T})^{G}\right)$ and $d_{2}\left(E_{2}^{1,2}\right)=0=$ $d_{3}\left(E_{3}^{2,1}\right)$, and by Proposition 6.9 we get the short exact sequence

$$
0 \rightarrow H^{3}\left(C^{*}(G, \mathbb{T})^{G}\right) \rightarrow H^{3}\left(\operatorname{Tot}\left(A^{*, *}\right)\right) \stackrel{\phi}{\rightarrow} M S_{G}(G) \rightarrow 0 .
$$


Moreover, the cokernel of the inclusion

$$
E_{\infty}^{0, q} \rightarrow H^{q}\left(\operatorname{Tot}\left(A^{*, *}\right)\right)
$$

should match the cohomology group $H^{q}\left(\operatorname{Tot}\left(B^{*, *}\right)\right)$ since this piece is built from the groups $E_{\infty}^{r, s}$ with $r, s>1$ and $r+s=p$, therefore we have the canonical isomorphism

$$
H^{q}\left(C^{*}(G, \mathbb{T})^{G}\right) \oplus H^{q}\left(\operatorname{Tot}\left(B^{*, *}\right)\right) \stackrel{\cong}{\rightrightarrows} H^{q}\left(\operatorname{Tot}\left(A^{*, *}\right)\right), x \oplus y \mapsto x+y
$$

which in particular implies that

$$
H^{q}\left(C^{*}(G, \mathbb{T})^{G}\right) \stackrel{\cong}{\longrightarrow} H^{q}(G, \mathbb{T}) .
$$

Then we can conclude that the composition of the maps

$$
H^{3}\left(\operatorname{Tot}\left(B^{*, *}\right)\right) \subset H^{3}\left(\operatorname{Tot}\left(A^{*, *}\right)\right) \stackrel{\phi}{\rightarrow} M S_{G}(G)
$$

is an isomorphism, and therefore we obtain the canonical isomorphism

$$
H^{3}\left(\operatorname{Tot}\left(B^{*, *}\right)\right) \stackrel{\cong}{\rightrightarrows} M S_{G}(G), \quad[\alpha \oplus \beta] \mapsto[\alpha \oplus \beta] .
$$

Also we obtain the short exact sequence

$$
0 \rightarrow H^{2}\left(C^{*}(G ; \mathbb{T})^{G}\right) \rightarrow H^{2}\left(\operatorname{Tot}\left(A^{*, *}\right)\right) \rightarrow H_{G}^{1}(G ; \mathbb{T})_{\text {mult }} \rightarrow 0,
$$

with $H_{G}^{1}(G ; \mathbb{T})_{\text {mult }} \cong H^{2}\left(\operatorname{Tot}\left(B^{*, *}\right)\right) \cong \operatorname{Hom}(G, \operatorname{Hom}(G, \mathbb{T}))$.

Now we will study the multiplicative structures that define the pseudomonoids constructed via the formalism introduced in [7], and whose properties were outlined in Section 4.5. For this purpose we need to calculate the composition of the maps

$$
H^{3}(G, \mathbb{T}) \stackrel{\tau_{1}^{*}}{\rightarrow} H^{3}\left(\operatorname{Tot}\left(A^{*, *}\right)\right) \stackrel{\phi}{\rightarrow} M S_{G}(G)
$$

where $\tau_{1}^{*}$ is the induced map in cohomology which was defined at the chain level in (3.2). This calculation will be carried out using the ring structure of the ring $H^{*}(G, \mathbb{Z})$ together with the pullback map $\mu^{*}: H^{*}(G, \mathbb{Z}) \rightarrow H^{*}(G \rtimes G, \mathbb{Z})$ induced by the multiplication $\mu: G \rtimes G \rightarrow G$ as we have in Theorem 3.3. Since we have the isomorphism

$$
H^{3}\left(\operatorname{Tot}\left(A^{*, *}\right)\right) \oplus H^{3}(G, \mathbb{T}) \stackrel{\cong}{\rightarrow} H^{3}(G \rtimes G, \mathbb{T}), \quad x \oplus y \mapsto x+\pi_{2}^{*} y
$$

where $\pi_{2}: G \rtimes G \rightarrow G,(a, g) \mapsto g$ is the projection, then the short exact sequence of (7.1) implies that the following is also a short exact sequence

$$
0 \rightarrow H^{3}\left(C^{*}(K ; \mathbb{T})^{G}\right) \oplus H^{3}(G, \mathbb{T}) \rightarrow H^{3}(G \rtimes G, \mathbb{T}) \rightarrow M S_{G}(G) \rightarrow 0 .
$$


We conclude that the homomorphism

$$
\phi \circ \tau_{1}^{*}: H^{3}(G, \mathbb{T}) \rightarrow M S_{G}(G)
$$

is equivalent to the composition of homomorphisms

$$
H^{3}(G, \mathbb{T}) \stackrel{\mu^{*}}{\rightarrow} H^{3}(G \rtimes G, \mathbb{T}) /\left(H^{3}\left(C^{*}(K ; \mathbb{T})^{G}\right) \oplus H^{3}(G, \mathbb{T})\right) \stackrel{\cong}{\rightarrow} M S_{G}(G) .
$$

Therefore we simply need to find the restriction to $H^{3}\left(\operatorname{Tot}\left(B^{*, *}\right)\right)$ of the image of $\mu^{*}$ in $H^{3}(G \rtimes G, \mathbb{T})$,

$$
H^{3}(G, \mathbb{T}) \stackrel{\mu^{*}}{\rightarrow} H^{3}(G \rtimes G, \mathbb{T}) \stackrel{\text { res }}{\rightarrow} H^{3}\left(\operatorname{Tot}\left(B^{*, *}\right)\right) \cong M S_{G}(G) .
$$

Let us see some examples.

7.2.1. Cyclic groups. Consider $G=K=\mathbb{Z} / n$ and note that as rings $H^{*}(G ; \mathbb{Z})=$ $\mathbb{Z}[x] /(n x)$ where $|x|=2$. By the Kunneth theorem we have that

$$
H^{2}(G \times G ; \mathbb{Z}) \cong H^{2}(G ; \mathbb{Z}) \otimes H^{0}(G ; \mathbb{Z}) \oplus H^{0}(G ; \mathbb{Z}) \otimes H^{2}(G ; \mathbb{Z})
$$

and moreover we have that

$$
\mu^{*} x=x \otimes 1+1 \otimes x
$$

Since $x^{2}$ is the generator of $H^{4}(G ; \mathbb{Z})$ we obtain

$$
\mu^{*} x^{2}=x^{2} \otimes 1+2 x \otimes x+1 \otimes x^{2}
$$

where

$$
H^{4}\left(\operatorname{Tot}\left(B^{*, *}(G \times G, \mathbb{Z})\right)\right)=\langle x \otimes x\rangle \cong \mathbb{Z} / n
$$

and therefore we obtain that the map

$$
\phi \circ \tau_{1}^{*}: H^{3}(G, \mathbb{T}) \rightarrow M S_{G}(G)
$$

is equivalent to the map

$$
H^{4}(G ; \mathbb{Z}) \rightarrow H^{4}\left(\operatorname{Tot}\left(B^{*, *}(G \times G, \mathbb{Z})\right)\right), x^{2} \mapsto 2 x \otimes x .
$$

Since in this case $H^{3}(G ; \mathbb{T}) \cong M S_{G}(G) \cong \mathbb{Z} / n$ we have that $\phi \circ \tau_{1}^{*}: \mathbb{Z} / n \stackrel{\times 2}{\rightarrow} \mathbb{Z} / n$.

Therefore, when $n$ is odd, the map $\phi \circ \tau_{1}^{*}$ is an isomorphism and therefore the Grothendieck rings of representations $\operatorname{Groth}\left(\operatorname{Rep}\left(D^{w}(G)\right)\right)$ of the Twisted Drinfeld Doubles $D^{w}(G)$ for $w \in H^{3}(G, \mathbb{T})$ are all non-isomorphic.

Meanwhile when $n$ is even, the cocycle $w \in Z^{3}(G, \mathbb{T})$ whose cohomology class is $\frac{n}{2} \in \mathbb{Z} / n \cong H^{3}(G, \mathbb{T})$ has for Grothendieck ring of representations 
$\operatorname{Groth}\left(\operatorname{Rep}\left(D^{w}(G)\right)\right)$ a ring isomorphic to $\operatorname{Groth}(\operatorname{Rep}(D(G)))$, which is by definition the ring $\mathbb{K} \mathbb{U}_{G}(G)=R(G) \otimes \mathbb{Z} G$. Moreover, the multiplicative structures defined by odd numbers in $\mathbb{Z} / n \cong M S_{G}(G)$ define Grothendieck rings of representations associated to the respective pseudomonoids which cannot be recovered via the Grothendieck ring of representations associated to the Twisted Drinfeld Double construction.

Note furthermore that in this case the automorphism groups are isomorphic

$$
H^{2}\left(\operatorname{Tot}\left(A^{*, *}(G \times G, \mathbb{T})\right)\right) \cong H_{G}^{1}(G, \mathbb{T})_{m u l t} \cong \mathbb{Z} / n .
$$

7.2.2. Quaternionic group. Consider $G=K=Q_{8}$ the quaternionic group and recall that $Q_{8} \subset S U(2)$ and that it sits in the short exact sequence

$$
0 \rightarrow \mathbb{Z} / 2 \rightarrow Q_{8} \rightarrow \mathbb{Z} / 2 \oplus \mathbb{Z} / 2 \rightarrow 0 .
$$

From the Serre fibration

$$
S U(2) / Q_{8} \rightarrow E S U(2) / Q_{8} \rightarrow B S U(2)
$$

one can deduce that the integral cohomology ring of $Q_{8}$ is

$$
H^{*}\left(Q_{8}, \mathbb{Z}\right)=\mathbb{Z}[x, y, e] /\left(x^{2}, y^{2}, x y, 2 x, 2 y, x e, y e, 8 e\right)
$$

with $|x|=|y|=2$ and $|e|=4$.

Since the cohomology of $Q_{8}$ is all of even degree, by the Kunneth isomorphism we have that

$$
H^{4}\left(Q_{8} \times Q_{8}, \mathbb{Z}\right) \cong \bigoplus_{j=0}^{4} H^{j}\left(Q_{8}, \mathbb{Z}\right) \otimes H^{4-j}\left(Q_{8}, \mathbb{Z}\right)
$$

and since $Q_{8} \times Q_{8} \cong Q_{8} \rtimes Q_{8}$ we have that

$$
H^{*}\left(Q_{8} \rtimes Q_{8}, \mathbb{Z}\right) \cong H^{*}\left(Q_{8} \times Q_{8}, \mathbb{Z}\right) .
$$

In this case we have that

$M S_{Q_{8}}\left(Q_{8}\right) \cong H^{4}\left(\operatorname{Tot}\left(B^{*, *}\left(Q_{8} \rtimes Q_{8}, \mathbb{Z}\right)\right)\right)=\langle x \otimes x, x \otimes y, y \otimes x, y \otimes y\rangle \cong(\mathbb{Z} / 2)^{\oplus 4}$

and since

$$
\mu^{*} e=e \otimes 1+1 \otimes e
$$

we can deduce that the map

$$
\phi \circ \tau_{1}^{*}: H^{3}\left(Q_{8}, \mathbb{T}\right) \stackrel{\times 0}{\rightarrow} M S_{Q_{8}}\left(Q_{8}\right)
$$

is the trivial map. 
A nice consequence of the triviality of the map $\phi \circ \tau_{1}^{*}$ is that for all $w \in$ $Z^{3}\left(Q_{8}, \mathbb{T}\right)$, the Grothendieck ring of representations of the $w$-Twisted Drinfeld Double is isomorphic as rings to $\mathbb{K} \mathbb{U}_{Q_{8}}\left(Q_{8}\right)$, which is the Grothendieck ring of representations of the untwisted Drinfeld double $D\left(Q_{8}\right)$.

Note also that the automorphism groups are isomorphic

$$
H^{2}\left(\operatorname{Tot}\left(A^{*, *}\left(Q_{8} \rtimes Q_{8}, \mathbb{T}\right)\right)\right) \cong H_{Q_{8}}^{1}\left(Q_{8}, \mathbb{T}\right)_{m u l t} \cong(\mathbb{Z} / 2)^{\oplus 4} .
$$

7.2.3. $G$ simple and non-abelian. In Section 4.5 .1 we showed that $H^{3}\left(\operatorname{Tot}\left(B^{*, *}(G \rtimes\right.\right.$ $G, \mathbb{T})))=0$ and therefore we have that $M S_{G}(G)=0$. Hence all Grothendieck rings of representations for all pseudomonoids are isomorphic to the ring $\mathbb{K} \mathbb{U}_{G}(G)$.

7.2.4. $G$ binary icosahedral. The same result applies to the binary icosehedral group since we showed in section 4.5.2 that $H^{3}\left(\operatorname{Tot}\left(B^{*, *}(G \rtimes G, \mathbb{T})\right)\right)=0$ and hence $M S_{G}(G)=0$.

7.3. $\mathbb{Z} / n$ acted by $\mathbb{Z} / 2$. Consider the action of $G=\mathbb{Z} / 2$ on $K=\mathbb{Z} / n$ given by multiplication of -1 . The group $\mathbb{Z} / n \rtimes \mathbb{Z} / 2$ is isomorphic to the dihedral group $D_{n}$ of rigid symmetries of the regular polygon of $n$ sides.

7.3.1. $n$ odd. Let us suppose that $n$ is odd and recall that in this case $H^{1}\left(D_{n}, \mathbb{T}\right)=$ $\mathbb{Z} / 2, H^{2}\left(D_{n}, \mathbb{T}\right)=0$ and $H^{3}\left(D_{n}, \mathbb{T}\right)=\mathbb{Z} / 2 \oplus \mathbb{Z} / n$. Since $H^{3}(\mathbb{Z} / 2, \mathbb{T})=\mathbb{Z} / 2$ we can conclude that $H^{3}\left(\operatorname{Tot}\left(A^{*, *}\right)\right)=\mathbb{Z} / n$. Now, applying the spectral sequence defined in Proposition 6.9 we have that $E_{1}^{1, q}=H_{G}^{1}\left(K^{q}, \mathbb{T}\right)=\mathbb{Z} / 2$, since the only fixed point of the $\mathbb{Z} / 2$ action is the $p$-tuple of zeros, and therefore we obtain that $E_{2}^{1, q}=0$ for $q>0$ since a simple calculation shows that the maps $d_{1}: E_{1}^{1,2 i-1} \rightarrow E_{1}^{1,2 i}$ are all isomorphisms. Moreover, since $E_{1}^{2, q}=$ $H_{G}^{2}\left(K^{q}, \mathbb{T}\right)=0$ because $H^{2}(\mathbb{Z} / 2, \mathbb{T})=0$ we have that $E_{3}^{2,1}=0$ and we can conclude that

$$
\operatorname{coker}\left(H^{3}\left(\operatorname{Tot}\left(A^{*, *}\right)\right) \stackrel{\phi}{\longrightarrow} M S_{G}(K)\right)=0 ;
$$

hence we have that the map $H^{3}\left(\operatorname{Tot}\left(A^{*, *}\right)\right) \stackrel{\phi}{\longrightarrow} M S_{G}(K)$ is surjective.

It remains now to calculate $\operatorname{ker}\left(H^{3}\left(\operatorname{Tot}\left(A^{*, *}\right)\right) \stackrel{\phi}{\longrightarrow} M S_{G}(K)\right)$. Applying the same argument as before we have that $E_{2}^{0,3}=H^{3}\left(C^{*}(K, \mathbb{T})^{G}\right)$ and we already know that $E_{2}^{1,2}=0=E_{2}^{2,1}$; therefore $\iota\left(H^{3}\left(C^{*}(K, \mathbb{T})^{G}\right)\right)$ coincides with the image of the canonical map $E_{2}^{0,3} \rightarrow H^{3}\left(\operatorname{Tot}\left(A^{*, *}\right)\right)$ and this map must be surjective. Therefore we have that

$$
\operatorname{ker}\left(H^{3}\left(\operatorname{Tot}\left(A^{*, *}\right)\right) \stackrel{\phi}{\longrightarrow} M S_{G}(K)\right)=\mathbb{Z} / n
$$


and we can conclude that

$$
M S_{G}(K)=0
$$

i.e. all multiplicative structures on the $\mathbb{Z} / 2$-equivariant $\mathrm{K}$-theory of $\mathbb{Z} / n$ are trivial and and all the Grothendieck rings $\operatorname{Groth}\left(\operatorname{Bun}_{\mathbb{Z} / 2}(\mathcal{K})\right)$ are isomorphic to the ring $\mathbb{K U}_{\mathbb{Z} / 2}(\mathbb{Z} / n)$ for any $\mathcal{K}=(\mathbb{Z} / n, m, 1, \alpha, \beta, \theta)$. $\mathbb{K U}_{\mathbb{Z} / 2}(\mathbb{Z} / n)$ is just the ring of isomorphism classes of representations of the dihedral group $D_{n}$.

In this case the automorphism groups are both trivial

$$
H^{2}\left(\operatorname{Tot}\left(A^{*, *}(\mathbb{Z} / n \rtimes \mathbb{Z} / 2, \mathbb{T})\right)\right)=0=H_{\mathbb{Z} / 2}^{1}(\mathbb{Z} / n, \mathbb{T})_{m u l t}
$$

7.3.2. $n$ even. Let us now suppose that $n$ is even; in this case $H^{1}\left(D_{n}, \mathbb{T}\right)=$ $\mathbb{Z} / 2 \oplus \mathbb{Z} / 2, H^{2}\left(D_{n}, \mathbb{T}\right)=\mathbb{Z} / 2$ and $H^{3}\left(D_{n}, \mathbb{T}\right)=\mathbb{Z} / 2 \oplus \mathbb{Z} / 2 \oplus \mathbb{Z} / n$. Since $H^{3}(\mathbb{Z} / 2, \mathbb{T})=\mathbb{Z} / 2$ we have that $H^{3}\left(\operatorname{Tot}\left(A^{*, *}\right)\right)=\mathbb{Z} / 2 \oplus \mathbb{Z} / n$. Now, we also have that

$$
E_{1}^{1, q}=H_{G}^{1}\left(K^{q}, \mathbb{T}\right) \cong \operatorname{Maps}\left((\mathbb{Z} / 2)^{q}, \mathbb{Z} / 2\right)
$$

since the fixed points of the $\mathbb{Z} / 2$ action on $K^{q}$ consists of $q$-tuples of points with either 0 or $\frac{n}{2}$ for entries. It is a simple calculation to show that the differential $d_{1}$ : $E_{1}^{1, i} \rightarrow E_{1}^{1, i+1}$ is precisely the differential of the cohomology of the group $\mathbb{Z} / 2$ with coefficients in $\mathbb{Z} / 2$ and therefore we get that

$$
E_{2}^{1, q} \cong H^{q}(\mathbb{Z} / 2, \mathbb{Z} / 2) \cong \mathbb{Z} / 2
$$

The groups $E_{1}^{2, q}$ are trivial because $H^{2}(\mathbb{Z} / 2, \mathbb{T})=0$.

Let us now calculate the group $E_{2}^{0,1}=H^{1}\left(C^{*}(K, \mathbb{T})^{G}\right)$. This group consists of the maps $f: \mathbb{Z} / n \rightarrow \mathbb{T}$ such that $f$ is invariant under the $G$-action, namely $f(x)=$ $f(-x)$, and that $\delta_{1} f=0$, which means that $f$ is a homomorphism. The only $G$-invariant homomorphisms are the ones that take values in the subgroup $\mathbb{Z} / 2 \subset \mathbb{T}$ and therefore we have that

$$
E_{2}^{0,1}=H^{1}\left(C^{*}(K, \mathbb{T})^{G}\right)=\mathbb{Z} / 2
$$


The information we have obtained so far on the cohomology groups of the second page of the spectral sequence is the following

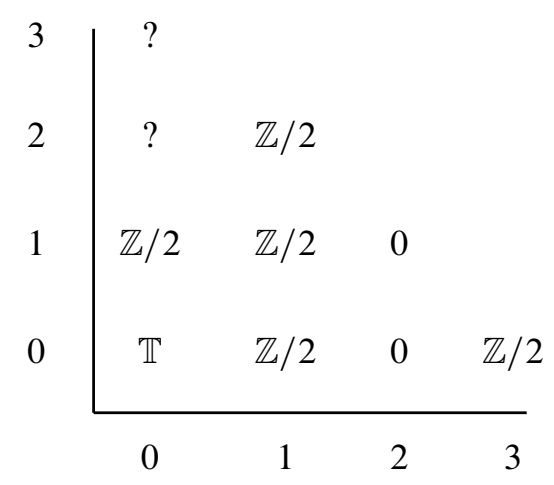

and since we know that $H^{1}\left(D_{n}, \mathbb{T}\right)=\mathbb{Z} / 2 \oplus \mathbb{Z} / 2$ and $H^{2}\left(D_{n}, \mathbb{T}\right)=\mathbb{Z} / 2$ we can deduce that $E_{\infty}^{0,2}=E_{2}^{0,2}=0$ and therefore $E_{2}^{0,3}=E_{4} 0,3=\mathbb{Z} / n$. Hence we have that

and

$$
\begin{array}{cl}
H^{3}\left(C^{*}(G, \mathbb{T})^{G}\right)=\mathbb{Z} / n, & M S_{G}(K)=\mathbb{Z} / 2 \\
H^{3}\left(\operatorname{Tot}\left(A^{*, *}\right)\right) \stackrel{\phi}{\longrightarrow} M S_{G}(K), & \mathbb{Z} / n \oplus \mathbb{Z} / 2 \rightarrow \mathbb{Z} / 2
\end{array}
$$

is the canonical projection map.

In this case the automorphism groups are isomorphic

$$
H^{2}\left(\operatorname{Tot}\left(A^{*, *}(\mathbb{Z} / n \rtimes \mathbb{Z} / 2, \mathbb{T})\right)\right) \cong H_{\mathbb{Z} / 2}^{1}(\mathbb{Z} / n, \mathbb{T})_{m u l t} \cong \mathbb{Z} / 2 .
$$

\section{A. Relation with (coquasi) bialgebras}

In what follows we will show how $B u n_{G}(\mathcal{K})$ can be understood as the tensor category of corepresentations associated to an explicit coquasi-bialgebra, and for this purpose we will show that the input necessary for defining such coquasi-bialgebra is equivalent to the information encoded in a pseudomonoid with strict unit in the 2-category of $G$-sets with twists.

\section{A.1. Coquasi-bialgebras.}

A.1.1. Coalgebras and comodules. Let $k$ be a field. A coalgebra over $k$ is a vector space over $k$ together with two linear maps $\Delta: C \rightarrow C \otimes C, \varepsilon: C \rightarrow k$ (called comultiplication and counit respectively) such that $(C \otimes \Delta) \Delta=(\Delta \otimes C) \Delta$ and $(C \otimes \varepsilon) \Delta=(\varepsilon \otimes C) \Delta=C$. We shall use the Sweedler's notation omitting the sum symbol, that is $\Delta(c)=c_{1} \otimes c_{2}$ if $c \in C$. 
If $C$ is a coalgebra, a right $C$-comodule is a vector $k$-space $M$ with a linear map $\rho: M \rightarrow M \otimes C$ such that $(\rho \otimes C) \rho=(M \otimes \Delta) \rho$ and $(M \otimes \varepsilon)=M$. Again for the comodule structure we shall use $S$ weedler's notation omitting the sum symbol, i.e., $\rho(m)=m_{0} \otimes m_{1}, m_{0} \in M, m_{1} \in C$. If $M, N$ are $C$-comodules, a comodule map is a linear map $f: M \rightarrow N$ such that $\rho_{N} f=(f \otimes C) \rho_{M}$. We shall denote by $\mathcal{M}^{C}$ the category of right $C$-comodules.

If $C, C^{\prime}$ are coalgebras $C \otimes C^{\prime}$ is a coalgebra with comultiplication $\Delta\left(c \otimes c^{\prime}\right)=$ $\left(c_{1} \otimes c_{1}^{\prime}\right) \otimes\left(c_{2} \otimes c_{2}^{\prime}\right)$ and counit $\varepsilon\left(c \otimes c^{\prime}\right)=\varepsilon(c) \varepsilon\left(c^{\prime}\right)$.

For a coalgebra $C$ the space $C^{*}$ is an associative algebra with the convolution product $f * g(c)=f\left(c_{1}\right) f\left(c_{2}\right)$ and unit $\varepsilon$.

A.1.2. Coquasi-bialgebras. A coquasi-bialgebra is a five-tuple $\left(H, \Delta, m, 1_{H}, \phi\right)$ where $H$ is a coassociative coalgebra with counit, $m: H \otimes H \rightarrow H, h \otimes g \mapsto h g$ is a coalgebra map, $1_{H}$ is a grouplike element (i.e. $\left.\Delta\left(1_{H}\right)=1_{H} \otimes 1_{H}\right)$ which is a unit for $m$, and $\phi \in(H \otimes H \otimes H)^{*}$ is a convolution invertible map (called the coassociator), satisfying the identities

$$
\begin{aligned}
\phi\left(g \otimes 1_{H} \otimes h\right) & =\varepsilon(g) \varepsilon(h), \\
m\left(m \otimes \mathrm{id}_{H}\right) * \phi & =\phi * m\left(\mathrm{id}_{H} \otimes m\right), \\
\phi\left(d_{1} f_{1} \otimes g_{1} \otimes h_{1}\right) \phi\left(d_{2} \otimes f_{2} \otimes\right. & \left.g_{2} h_{2}\right) \\
& =\phi\left(d_{1} \otimes f_{1} \otimes g_{1}\right) \phi\left(d_{2} \otimes f_{2} g_{2} \otimes h_{1}\right) \phi\left(f_{1} \otimes g_{3} \otimes h_{2}\right)
\end{aligned}
$$

for all $f, g, h \in H$.

The category of right $H$-comodules $\mathcal{M}^{H}$ is monoidal, where the tensor product is over the base field and the comodule structure of the tensor product is induced by the multiplication. The associator is given by

$$
\begin{array}{r}
\Phi_{U, V, W}:(U \otimes V) \otimes W \longrightarrow U \otimes(V \otimes W) \\
\Phi_{U, V, W}((u \otimes v) \otimes w)=\phi^{-1}\left(u_{1}, v_{1}, w_{1}\right) u_{0} \otimes\left(v_{0} \otimes w_{0}\right)
\end{array}
$$

for $u \in U, v \in V, w \in W$ and $U, V, W \in \mathcal{M}^{H}$.

A.2. Coquasi-bialgebras associated to pseudomonoids. Let $G$ be a finite group and $\mathcal{K}=(K, m, 1, \alpha, \beta, \theta)$ a pseudomonoid with strict unit in the 2-category of $G$-sets with twists; let us denote $\mathbb{C}^{G}:=\operatorname{Maps}(G, \mathbb{C})$, let $\delta_{\sigma} \in \mathbb{C}^{G}$ be the function that assigns 1 to $\sigma$ and 0 otherwise, and let $\delta_{\sigma, \tau}$ be the Dirac's delta associated to the pair $\sigma, \tau \in G$, namely $\delta_{\sigma, \tau}$ is 1 whenever $\sigma=\tau$ and 0 otherwise.

Theorem A.1. The vector space $\mathbb{C}^{G} \# K$ with basis $\left\{\delta_{\sigma} \# x \mid \sigma \in G, x \in K\right\}$ is a coquasi-bialgebra with product

$$
\left(\delta_{\sigma} \# x\right)\left(\delta_{\tau} \# y\right)=\delta_{\sigma, \tau} \beta[\sigma|| x \mid y] \delta_{\sigma} \# x y,
$$


coproduct,

$$
\Delta\left(\delta_{\sigma} \# x\right)=\sum_{a, b \in G: a b=\sigma} \alpha[a|b| \mid x] \delta_{a} \# b x \otimes \delta_{b} \# x,
$$

associator

$$
\phi\left(\delta_{\sigma} \# x, \delta_{\tau} \# y, \delta_{\rho} \# z\right)=\delta_{\sigma, e} \delta_{\tau, e} \delta_{\rho, e} \theta[x|y| z],
$$

counit $\varepsilon\left(\delta_{\sigma} \# x\right)=\delta_{\sigma, e}$, and unit $1 \#$ for all $\sigma, \tau, \rho \in G, x, y, z \in K$.

Moreover, the tensor category of right $\mathbb{C}^{G} \# \mathbb{C} K$-comodules is tensor isomorphic to the monoidal category $\mathrm{Bu}_{G}(\mathcal{K})$ of equivariant vector bundles on $\mathcal{K}$.

Proof. It is straightforward to check that $\mathbb{C}^{G} \# K$ satisfies all axioms of coquasibialgebra.

If $V=\bigoplus_{x \in K} V_{x}$ is an object in $B u n_{G}(\mathcal{K})$, we define a right $\mathbb{C}^{G} \# \mathbb{C} K$-comodule structure over $V$ by

$$
\rho\left(v_{x}\right)=\sum_{\sigma \in G} \sigma \triangleright v_{x} \otimes \delta_{\sigma} \# x,
$$

for all $v_{x} \in V_{x}, x \in K$. It follows that this rule defines a strict monoidal functor $\mathcal{F}$ from $\operatorname{Bun}_{G}(\mathcal{K})$ to the category of right $\mathbb{C}^{G} \# \mathbb{C} K$-comodules.

Conversely, if $(V, \rho)$ is a right $\mathbb{C}^{G} \# \mathbb{C} K$-comodule then

$$
V=\bigoplus_{x \in K} V_{x}, \quad \text { where } V_{x}=\{v \in V:(i d \otimes \pi) \rho=v \otimes x\},
$$

where $\pi: \mathbb{C}^{G} \# K \rightarrow \mathbb{C} K, \delta_{\sigma} \# x \mapsto \delta_{\sigma, e} u_{x}$ and the comodule map defines unique linear maps $\triangleright: \mathbb{C} G \otimes V_{x} \rightarrow V(x \in G)$ such that

$$
\rho\left(v_{x}\right)=\sum_{\sigma \in G} \sigma \triangleright v_{x} \# x,
$$

for all $v_{x} \in V_{x}$. It follows that $\left(V=\bigoplus_{x \in K} V_{x}, \triangleright\right) \in \operatorname{Bun}_{G}(\mathcal{K})$, and this rule defines a functor that by construction is the inverse of $\mathcal{F}$.

Remark A.2. If $\theta$ is trivial, $\mathbb{C}^{G} \# K$ is a Hopf algebra, and if we consider $G$ acting by conjugation on $K=G$ together with the 3-cocycle $w \in Z^{3}(G, \mathbb{T})$, the coquasi-bialgebra $\mathbb{C}^{G} \# \mathbb{C} G$ defined by the pseudomonoid $\left(G, m, 1, \alpha_{w}, \beta_{w}, \theta_{w}\right)$ as in Section 4.5, is the dual of the Twisted Drinfeld Double of the finite group $G$ defined in [7, Section 3.2].

We finish with a corollary of the results of this appendix and the ones of Section 7.2. A quasi-isomorphism between coquasi- bialgebras $(H, \psi)$ and $\left(H^{\prime}, \psi\right)$ is a pair $(F, \theta)$ consisting of a coalgebra isomorphism $F: H \rightarrow H^{\prime}$ and a convolution invertible map $\theta \in(H \otimes H)^{*}$ such that $\theta(1 \otimes h)=\theta(h \otimes 1)=\varepsilon(h)$ and

$$
\theta\left(g_{1} \otimes h_{1}\right) F\left(g_{2} h_{2}\right)=F\left(g_{1}\right) F\left(h_{2}\right) \theta\left(g_{2} \otimes h_{2}\right),
$$


for all $g, h \in H$. A quasi-isomorphism is called an isomorphism of coquasibialgebras if additionally

$$
\begin{aligned}
\theta\left(f_{1} \otimes g_{2}\right) \theta\left(f_{2} g_{2} \otimes h_{1}\right) \psi\left(f_{3} \otimes g_{3} \otimes h_{3}\right) & \\
& =\psi^{\prime}\left(F\left(f_{1}\right) \otimes F\left(g_{1}\right) \otimes F\left(h_{1}\right)\right) \theta\left(g_{2} \otimes h_{2}\right) \theta\left(f_{2} \otimes g_{3} h_{3}\right) .
\end{aligned}
$$

In general, the tensor category of representations of $D^{w}(G)$ is not equivalent to the category of representations of any Hopf algebra. However, by the isomorphism outlined in (7.2), if $G$ is finite and acts over itself by conjugation, the Grothendieck ring of $\operatorname{Bun}_{G}(\mathcal{K})$ for any 3-cocycle in $Z^{3}\left(\operatorname{Tot}^{*}\left(A^{* * *}(G \rtimes G, \mathbb{T})\right)\right.$ ), is always equivalent to the Grothendieck ring of $B u n_{G}\left(\mathcal{K}^{\prime}\right)$, where the 3-cocycle associated lives in $Z^{3}\left(\operatorname{Tot}^{*}\left(B^{*, *}(G \rtimes G, \mathbb{T})\right)\right)$. By Theorem A.1, $B u n_{G}\left(\mathcal{K}^{\prime}\right)$ is the category of representation of a Hopf algebra, so in particular we can conclude

Corollary A.3. The Twisted Drinfeld Double of a finite group is always quasiisomorphic to a Hopf algebra.

\section{References}

[1] A. Adem and R. J. Milgram. Cohomology of finite groups, Grundlehren der Mathematischen Wissenschaften [Fundamental Principles of Mathematical Sciences], 309. Springer-Verlag, Berlin, 1994. Zbl 0820.20060 MR 1317096

[2] A. Adem, Y. Ruan, and B. Zhang. A stringy product on twisted orbifold Ktheory. Morfismos, 11 (2007), no. 2.

[3] N. Bárcenas, J. Espinoza, M. Joachim, and B. Uribe. Universal twist in equivariant K-theory for proper and discrete actions. Proc. London Math. Soc., 108 (2014), no. 5, 1313-1350. Zbl 1297.19008 MR 3214681

[4] E. Becerra and B. Uribe. Stringy product on twisted orbifold K-theory for abelian quotients. Trans. Amer. Math. Soc., 361 (2009), no. 11, 5781-5803. Zbl 1191.14068 MR 2529914

[5] F. Borceux. Handbook of categorical algebra. 2, Encyclopedia of Mathematics and its Applications, 51. Cambridge University Press, Cambridge, 1994. Zbl 0843.18001 MR 1313497

[6] K. S. Brown. Cohomology of groups, Graduate Texts in Mathematics, 87. Springer-Verlag, New York, 1982. Zbl 0584.20036 MR 672956

[7] R. Dijkgraaf, V. Pasquier, and P. Roche. Quasi Hopf algebras, group cohomology and orbifold models. Recent advances in field theory (Annecyle-Vieux, 1990). Nuclear Phys. B Proc. Suppl. 18B (1990), (1991), 60-72. Zbl 0957.81670 MR 1128130 
[8] S. Eilenberg and S. Mac Lane. On the groups of $H(\Pi, n)$. I. Ann. of Math. (2), 58 (1953), 55-106. Zbl 0050.39304 MR 56295

[9] P. Etingof, D. Nikshych, and V. Ostrik. On fusion categories. Ann. of Math. (2), 162 (2005), no. 2, 581-642. Zbl 1125.16025 MR 2183279

[10] P. J. Hilton and U. Stammbach. A course in homological algebra, Graduate Texts in Mathematics, 4, second edition. Springer-Verlag, New York, 1997. Zbl 0863.18001 MR 1438546

[11] J. Hu and B.-L. Wang. Delocalized Chern character for stringy orbifold K-theory. Trans. Amer. Math. Soc., 365 (2013), no. 12, 6309-6341. Zbl 1278.19011 MR 3105753

[12] G. I. Kac. Group extensions which are ring groups. Mat. Sb. (N.S.), 76 (1968), no. 118, 473-496. MR 229061

[13] G. Karpilovsky. The Schur multiplier, London Mathematical Society Monographs, New Series, 2. The Clarendon Press, Oxford University Press, New York, 1987. Zbl 0619.20001 MR 1200015

[14] A. Masuoka. Hopf algebra extensions and cohomology in New directions in Hopf algebras, Math. Sci. Res. Inst. Publ., 4, 167-209. Cambridge Univ. Press, Cambridge, 2002. Zbl 1011.16024 MR 1913439

[15] A. Masuoka. Cohomology and coquasi-bialgebra extensions associated to a matched pair of bialgebras. Adv. Math., 173 (2003), no. 2, 262-315. Zbl 1026.16023 MR 1957830

[16] C. C. Moore. Group extensions and cohomology for locally compact groups. III. Trans. Amer. Math. Soc., 221 (1976), no. 1, 1-33. Zbl 0366.22005 MR 414775

[17] V. Ostrik. Module categories, weak Hopf algebras and modular invariants. Transform. Groups, 8 (2003), no. 2, 177-206. Zbl 1044.18004 MR 1976459

[18] V. G. Turaev. Quantum invariants of knots and 3-manifolds, de Gruyter Studies in Mathematics, 18, revised edition. Walter de Gruyter \& Co., Berlin, 2010. Zbl 1213.57002 MR 2654259

[19] S. Willerton. The twisted Drinfeld double of a finite group via gerbes and finite groupoids. Algebr. Geom. Topol., 8 (2008), no. 3, 1419-1457. Zbl 1154.57029 MR 2443249

[20] S. J. Witherspoon. Products in Hochschild cohomology and Grothendieck rings of group crossed products. Adv. Math., 185 (2004), no. 1, 136-158. Zbl 1063.16012 MR 2058782 
Received 05 February, 2014; revised 15 July, 2014

C. Galindo, Departamento de Matemáticas, Universidad de los Andes, Carrera 1 N. 18A - 10, Bogotá 111711, Colombia

E-mail: cn.galindo1116@uniandes.edu.co; cesarneyit@gmail.com

I. Gutiérrez, Departamento de Matemáticas, Universidad del Norte, Km.5 Vía Puerto Colombia, Barranquilla 3509509, Colombia

E-mail: isgutier@uninorte.edu.co

B. Uribe, Departamento de Matemáticas, Universidad del Norte, Km.5 Vía Puerto Colombia, Barranquilla 3509509, Colombia

E-mail: buribe@gmail.com; bjongbloed@uninorte.edu.co 